\title{
Effects of feed oil content, transmembrane pressure and membrane rotational speed on permeate water quality in high- shear rotary ultrafiltration
}

\author{
David Albert Masciola \\ West Virginia University
}

Follow this and additional works at: https://researchrepository.wvu.edu/etd

\author{
Recommended Citation \\ Masciola, David Albert, "Effects of feed oil content, transmembrane pressure and membrane rotational \\ speed on permeate water quality in high-shear rotary ultrafiltration" (1999). Graduate Theses, \\ Dissertations, and Problem Reports. 971. \\ https://researchrepository.wvu.edu/etd/971
}

This Thesis is protected by copyright and/or related rights. It has been brought to you by the The Research Repository @WVU with permission from the rights-holder(s). You are free to use this Thesis in any way that is permitted by the copyright and related rights legislation that applies to your use. For other uses you must obtain permission from the rights-holder(s) directly, unless additional rights are indicated by a Creative Commons license in the record and/ or on the work itself. This Thesis has been accepted for inclusion in WVU Graduate Theses, Dissertations, and Problem Reports collection by an authorized administrator of The Research Repository @ WVU. For more information, please contact researchrepository@mail.wvu.edu. 


\title{
EFFECTS OF FEED OIL CONTENT, TRANSMEMBRANE PRESSURE AND MEMBRANE ROTATIONAL SPEED ON PERMEATE WATER QUALITY IN HIGH-SHEAR ROTARY ULTRAFILTRATION
}

\author{
David A. Masciola
}

\begin{abstract}
Thesis submitted to the
at West Virginia University

for the degree of

Master of Science
in
Civil Engineering
\end{abstract} College of Engineering and Mineral Resources

in partial fulfillment of the requirements

Brian E. Reed, Ph.D., Chair

Roger C. Viadero Jr., Ph.D.

David Martinelli, Ph.D.

Department of Civil Engineering

Morgantown, West Virginia

1999

Keywords: Membrane Separation, Ultrafiltration, Oil-In-Water Emulsions Copyright 1999 David A. Masciola 
TABLE OF CONTENTS

TITLE PAGE

TABLE OF CONTENTS _.................................................................. ii iv

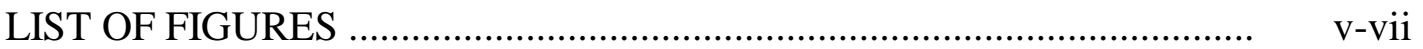

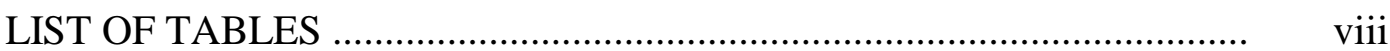

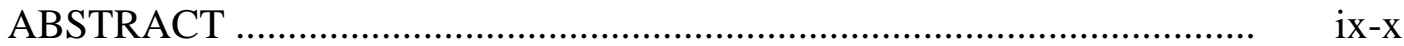

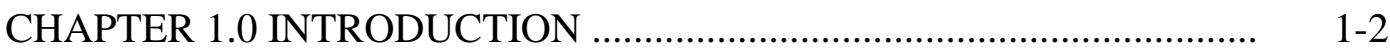

CHAPTER 2.0 BACKGROUND …………………............................

MEMBRANE SEPARATION PROCESSES ................................... 3-. 3-38

General Process Description ................................................ 3-5

Operational Modes ……………………........................ $5-8$

Recycle Mode ………………………………….... 6

Semi-Batch Mode ……………………………..... $\quad$ 6-8

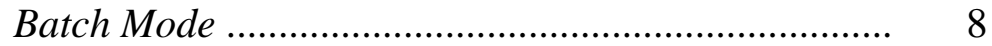

General Types of Membrane Separation Processes ………...... 8-13

Reverse Osmosis and Nanofiltration ......................... $\quad 8-12$

Ulltrafiltration and Microfiltration ............................ 12-13

Configurations of Conventional Membrane Modules 13-20

Tubular Membrane Modules ...................................... 13-15

Hollow Membrane Fiber Modules .............................. 15-17

Spiral Wound Membrane Modules ............................. $\quad 17-19$

Plate and Frame Membrane Modules ....................... 19. 19-20

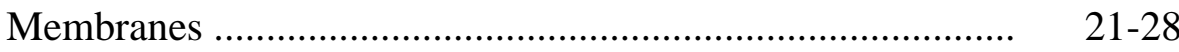

Factors Controlling Permeate Flux ………………………..... 28-35

Concentration Polarization ………............................ 28-33

Membrane Fouling ………...................................... $\quad 33-35$

Limitations of Conventional Cross-Flow Ultrafiltration

Systems .................................................................. 38

MODELING PERMEATE FLUX ……………………………....... 39-63

Pressure-Controlled Region--Hagen-Poiseuille Model .......... 39-42

The Osmotic Pressure Model .................................................. 42-43 
The Resistance Model ........................................................... 43

Mass Transfer-Controlled Region-The Thin-Film Model $\quad 44$

Mechanically Enhanced UF Systems $44-63$ Flow Conditions in Rotary Membrane Systems ........... 48-55

Permeate Backpressure In A High-Shear Rotary UF

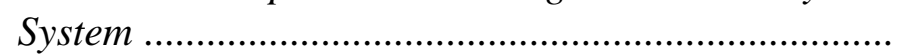
Mechanically Enhanced Ultrafiltration Case Studies

MEMBRANE TREAT MENT OF OILY WASTEWATERS $63-76$

Characteristics of Oil-In-Water (O/W) Emulsions And Oily

Wastewaters 63-69

Overview of Membrane Treatment of Oily Wastewaters 69-76 Conventional Ultrafiltration Systems . 69-76 Mechanically Enhanced Ultrafiltration Systems $72-76$

CHAPTER 3.0 INVESTIGATIVE APPROACH

$77-93$

EXPERIMENTAL DESIGN $77-81$

MATERIALS AND METHODS $81-93$

Experimental Apparatus $81-85$

Experimental Conduct $86-92$

Membrane Cleaning Procedure 86- 89

Metal-Working Fluid Experiments $89-92$

Feed Preparation $89-90$

System Operation 90-92

System Shutdown 92 Analytical Methods 92-93

Oil and Grease 92-93

Total Organic Carbon

93 Quality Assurance/Quality Control

93

CHAPTER 4.0 RESULTS

94-113

5\% MW Fluid -1750 rpm

94-95

5\% MW Fluid -1150 rpm 96

10\% MW Fluid -1750 rpm 96-99 10\% MW Fluid -1150 rpm 99 15\% MW Fluid -1750 rpm 99-100 15\% MW Fluid -1150 rpm 100 20\% MW Fluid - 1750 rpm 100-104 20\% MW Fluid -1150 rpm 104 
30\% MW Fluid -1750 rpm .............................................. 107

30\% MW Fluid -1150 rpm ............................................... 107-110

40\% MW Fluid $-1750 \mathrm{rpm}$.............................................. 110

40\% MW Fluid -1150 rpm ............................................. 110 1

CHAPTER 5.0 DISCUSSION ............................................................... 114-121

CHAPTER 6.0 CONCLUSIONS ........................................................ 122

CHAPTER 7.0 REFERENCES ........................................................... 123-128 


\section{LIST OF FIGURES}

Figure (2.1) General Membrane Separation Process.

Figure (2.2) A Schematic of Semi-Batch and Batch Operation Modes.

Figure (2.3) The Useful Ranges of the Four General Membrane Separation $\quad 9$ Processes (After Cheryan (1986) and Perry and Green (1997)).

Figure (2.4) The Phenomena of Osmosis and Reverse Osmosis 11

$\begin{array}{lll}\text { Figure (2.5) A Schematic of a Tubular Membrane Module. } & 14\end{array}$

Figure (2.6) A Schematic of a Hollow Fiber Membrane Module. 16

$\begin{array}{lll}\text { Figure (2.7) } & \text { A Schematic of a Spiral Wound Membrane Module. } & 18\end{array}$

Figure (2.8) A Schematic of a Plate and Frame Membrane Module. 20

Figure (2.9) The Relationship Between Permeate Flux and Transmembrane 23 Pressure for a Clean Water Feed.

Figure (2.10) A Schematic of the Passage of an Oil Droplet Through the Pores 26 of a Membrane.

Figure (2.11) The Theoretical Relationship Between Permeate Flux, 29 Transmembrane Pressure, Feed Concentration, and Hydraulic Turbulence.

Figure (2.12) A Schematic of the Three Primary Solute Transport Mechanisms 30 in Membrane Separation Systems.

Figure (2.13) A Schematic of Solute Molecules at the Membrane Surface 36

Figure (2.14) A Schematic of a Typical Concentric Cylinder Module. 46

Figure (2.15) A Schematic of a High-Shear Rotary UF Pressure Vessel 49

Figure (2.16) Illustration of "Conditioning Shuttles." $\quad 50$ 
Figure (2.17) The Membrane Radius and Spacing in a High-Shear Rotary UF 53 System.

Figure (2.18) A Schematic of an Emulsified Oil Droplet 66

Figure (3.1) A Schematic of the Membrane Vessel 83

Figure (3.2) A Schematic of the Experimental Apparatus 84

Figure (4.1) Permeate Oil and Grease and Total Organic Carbon Versus 95 Average Transmembrane Pressure For the 5\% MWF-1750 rpm Combination.

Figure (4.2) Permeate Oil and Grease and Total Organic Carbon Versus Average Transmembrane Pressure For the 5\% MWF-1150 rpm Combination.

Figure (4.3) Permeate Oil and Grease and Total Organic Carbon Versus Average Transmembrane Pressure For the 10\% MWF-1750 rpm Combination.

Figure (4.4) Permeate Oil and Grease and Total Organic Carbon Versus Average Transmembrane Pressure For the 10\% MWF-1150 rpm Combination.

Figure (4.5) Permeate Oil and Grease and Total Organic Carbon Versus Average Transmembrane Pressure For the 15\% MWF-1750 rpm Combination.

Figure (4.6) Permeate Oil and Grease and Total Organic Carbon Versus 104 Average Transmembrane Pressure For the 15\% MWF-1150 rpm Combination.

Figure (4.7) Permeate Oil and Grease and Total Organic Carbon Versus 106 Average Transmembrane Pressure For the 20\% MWF-1750 rpm Combination.

Figure (4.8) Permeate Oil and Grease and Total Organic Carbon Versus Average Transmembrane Pressure For the 20\% MWF-1150 rpm Combination. 
Figure (4.9) Permeate Oil and Grease and Total Organic Carbon Versus Average Transmembrane Pressure For the 30\% MWF-1750 rpm Combination.

Figure (4.10) Permeate Oil and Grease and Total Organic Carbon Versus Average Transmembrane Pressure For the 30\% MWF-1150 rpm Combination.

Figure (4.11) Permeate Oil and Grease and Total Organic Carbon Versus Average Transmembrane Pressure For the 40\% MWF-1750 rpm Combination.

Figure (4.12) Permeate Oil and Grease and Total Organic Carbon Versus Average Transmembrane Pressure For the 40\% MWF-1150 rpm Combination.

Figure (5.1) Permeate Oil and Grease Versus Feed Oil Content for the 1750 rpm-25, 55, and 70 psig Combinations.

Figure (5.2) Permeate Total Organic Carbon Versus Feed Oil Content for the 1750 rpm-35, 65, and 70 psig Combinations.

Figure (5.3) MW Fluid Absolute Viscosity Versus \% Metal-Working Fluid

Figure (5.4) Permeate Oil and Grease Versus Average Transmembrane Pressure For the $40 \%$ MWF Combinations.

Figure (5.5) Permeate Total Organic Carbon Versus Average Transmembrane 122 Pressure For the $40 \%$ MWF Combinations. 


\section{LIST OF TABLES}

TABLE

PAGE

Table (2.1) Typical Membrane Resistances for Micro and Ultrafiltration

Membranes.

Table (2.2) Viscosity of Water at Several Common Operating Temperatures

Table (2.3) Typical O/G Concentration Ranges for Selected Industrial

64 Wastewaters.

Table (3.1) Metal-Working Fluid/Membrane Rotational Speed

Combinations and Pressure Ranges Investigated in this Study.

Table (3.2) Average and Maximum Permeate Backpressure at Typical

Rotational Speeds.

Table (3.3) A Summary of the Ceramic Membrane Characteristics.

82

Table (3.4) A Summary of the Characteristics of Base/Surfactant and Acid Cleaning Solutions Used in the Standard Cleaning Procedure.

Table (3.5) The Base MW Package and Water Volumes Added to Make Each MW Fluid Concentration. 


\title{
ABSTRACT \\ EFFECTS OF FEED OIL CONTENT, TRANSMEMBRANE PRESSURE AND MEMBRANE ROTATIONAL SPEED ON PERMEATE WATER QUALITY IN HIGH-SHEAR ROTARY ULTRAFILTRATION
}

\author{
By David A. Masciola
}

The metal-working industry produces large volumes of oily wastewater used in grinding, machining and rolling mill operations. The ability to treat oily wastes using conventional membrane modules (e.g. tubular and hollow fiber) is limited due to an inability to induce sufficient turbulence at the membrane surface as wastes are concentrated. In the high-shear rotary ultrafiltration (HSRUF) unit, disk membranes are rotated at speeds up to $1,750 \mathrm{rpm}$ to generate hydraulic turbulence which scours the membrane surface. The pump is required only to provide transmembrane pressure and a small amount of feed recirculation. Little data is available on the effects of operating parameters on permeate water quality; thus, a study was conducted to investigate the effects of transmembrane pressure, membrane rotational speed and feed oil content on permeate water quality in HSRUF treatment of a synthetic metal working fluid. The results from this study, combined with those of previous studies focusing primarily on permeate flux, can be extremely helpful in determining more efficient operating conditions.

Experiments were conducted using a HSRUF system equipped with a $0.11 \mu \mathrm{m}$ average pore size ceramic membrane. Experiments at a single operating temperature of $11{ }^{\circ} \mathrm{F}$ were conducted at discrete rotational speed/metal-working fluid concentration combinations over an applied pressure range of 25 to 75 psig and membrane rotational speeds of 1150 and $1750 \mathrm{rpm}$. Synthetic solutions containing 5 to $40 \%$ metal-working fluid were prepared in each experiment. 
Permeate oil and grease and total organic carbon increased with increasing transmembrane pressure and feed oil content due to an increase in the thickness and density of the solute concentration boundary layer. However, the hydraulic turbulence created by membrane rotation minimized the thickness of the solute concentration boundary layer therefore reducing solute permeation through the membrane. The effective decoupling of feed pressurization/recirculation from hydraulic turbulence enabled the HSRUF system to provide a better permeate quality in the treatment of highly concentrated oily wastes. 


\section{CHAPTER 1.0 INTRODUCTION}

The metal-working industry produces large volumes of wastewater in the form of waste emulsified oils used in grinding, machining and rolling mill operations. Waste emulsions containing approximately $0.5 \%$ oil and grease $(\mathrm{O} / \mathrm{G})$ are treated using a number of different techniques, including; chemical addition, dissolved air floatation, acid or heat cracking and gravity separation. Recently, membrane separation processes such as ultrafiltration (UF) and microfiltration (MF) have emerged as more efficient treatment technologies.

As wastes are concentrated, increases in viscosity make it difficult to maintain high cross-flow velocities, therefore hydraulic turbulence at the membrane surface decreases. The buildup of rejected solute molecules near the membrane surface is augmented by decreased hydraulic turbulence, and is defined as concentration polarization. The ability to treat oily wastes using conventional membrane modules (e.g. tubular and hollow fiber) is limited due to an inability to induce sufficient turbulence at the membrane surface as wastes are concentrated. In high-shear rotary UF (HSRUF), hydraulic turbulence is decoupled from feed recirculation/pressurization. Disk membranes are rotated at speeds up to $1750 \mathrm{rpm}$ to induce hydraulic turbulence that scours the membrane surface; therefore it is not necessary to maintain high cross-flow velocities.

Presently, little is known about the relationship between operating parameters and permeate water quality in HSRUF treatment of oily wastes. Therefore, a parametric, 
waste-specific study was conducted to provide insight on the relationship between feed oil concentration, membrane rotational speed, and transmembrane pressure on permeate water quality in HSRUF treatment of a synthetic metal working fluid (MWF). 


\section{CHAPTER 2.0 \\ BACKGROUND}

\section{MEMBRANE SEPARATION PROCESSES}

In this section, an overview of the main characteristics; operating modes; types of membrane separation systems; and configurations of conventional membrane modules are presented. Additionally, the factors which control system operation and the limitations of conventional membrane separation systems are discussed.

\section{General Process Description}

Membrane filtration is a fluid/fluid and particle/fluid separation technology used to separate dissolved substances and fine particles from water and wastewater. A general membrane separation process is shown schematically in Figure (2.1). A thin semipermeable membrane acts as a selective barrier that separates particles based on molecular or physical size. In general, water and constituents which are smaller than the membrane pore size are able to pass through the membrane as "permeate" while particles and molecules larger than the membrane pore size are retained. The retained particles and molecules, known as "concentrate," "retentate," or "residual," are returned to the feed tank to be concentrated further with time. "Permeate flux" is the term used to describe how fast permeate passes through a membrane. Flux is generally reported as volume/area-time. Permeate flux generally depends upon: the individual membrane characteristics (e.g., membrane pore size and membrane surface charge) the characteristics of the feed stream (e.g., viscosity and solute particle size); and operating parameters (e.g., transmembrane pressure and feed temperature). 


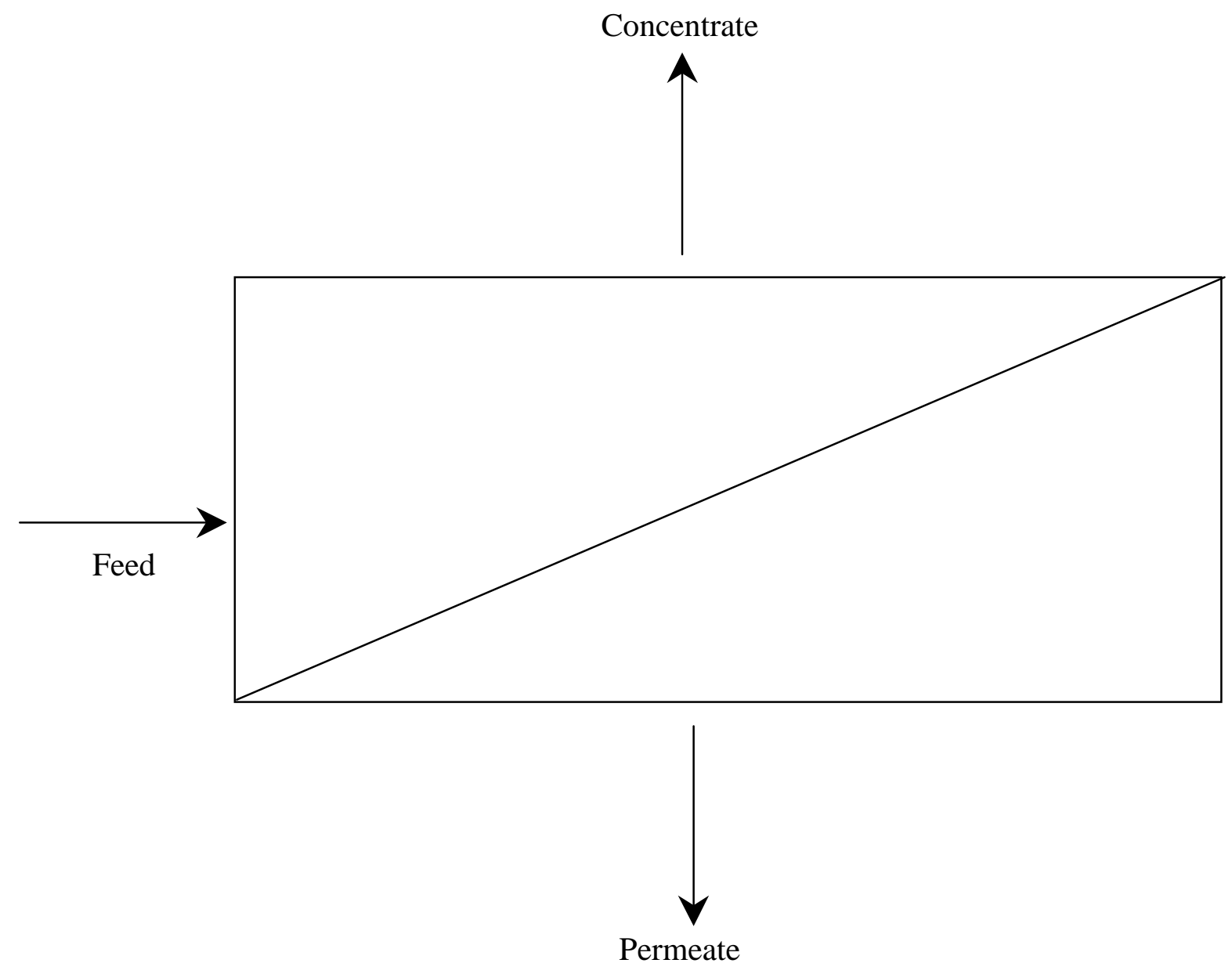

Figure (2.1)—General Membrane Separation Process. 
Most membrane separation systems used in industrial applications are operated in a cross-flow feed configuration as opposed to dead-end mode. In the cross-flow configuration, concentrate passes parallel to the membrane surface as opposed to perpendicular flow which is used in dead-end filtration. The accumulation of solute molecules at the membrane surface is decreased and the subsequent loss of permeate flux due to increased hydrodynamic resistance at the membrane surface is minimized by crossflow induced hydraulic turbulence.

Membrane separation processes are gaining acceptance as viable alternatives to other treatment technologies such as dissolved air flotation and biological treatment. For example, membrane separation systems can be used to recover valuable materials from waste streams (e.g., reusable oil, metal salts, etc.) and to reduce the volume of waste sent for further treatment or disposal. Additional industrial applications of membrane separation systems include: metal-working wastewaters, pulp and paper wastewaters, commercial laundry wastewaters, landfill leachates, the production of high quality water used in the production of microelectronic circuits, and in beverage processing.

\section{Operational Modes}

Membrane separation systems are typically operated in one of three main filtration modes: (1) recycle mode, (2) semi-batch ("modified batch") mode, or (3) batch mode. Since membrane separation technologies are volume reduction processes, the concentration factor $(\mathrm{CF})$ is an important system efficiency parameter. The concentration factor is defined as the ratio of feed concentration at a given time to the initial concentration. Concentration factors are typically expressed as $1 \mathrm{X}, 2 \mathrm{X}$, etc., and increase 
with treatment time. Concentration factors in excess of 100 are possible with membrane separation technologies.

\section{Recycle Mode}

In recycle mode, feed is pumped from the feed tank into the membrane module. Permeate is forced through the membrane under pressure and is returned to the feed tank. Thus, the concentration of the solution in the feed tank remains constant over time. Since the feed solution is not concentrated over time, recycle mode is not used in industrial waste and process applications. However, membrane separation systems are frequently operated in recycle mode in laboratory studies when it is advantageous to maintain a constant feed concentration.

\section{Semi-Batch Mode}

In field applications, membrane separation systems are typically operated in either semi-batch or batch mode. A schematic of semi-batch and batch operation modes is presented in Figure (2.2). In semi-batch mode, fresh feed solution (at 1X) is added to the feed tank at the same rate permeate is produced and the feed solution is concentrated over time. The concentration of the feed solution in semi-batch operation is given in Equation (2.1).

$$
\mathrm{CF}_{\mathrm{sb}}=1+\mathrm{V}_{\text {perm }} / \mathrm{V}_{\text {feed }}
$$

where $\mathrm{CF}_{\mathrm{sb}}=$ concentration factor during semi-batch operation, $\mathrm{V}_{\text {perm }}=$ volume of permeate produced, and $\mathrm{V}_{\text {feed }}=$ volume of solution in feed tank (constant during semi- 
SEMI-BATCH

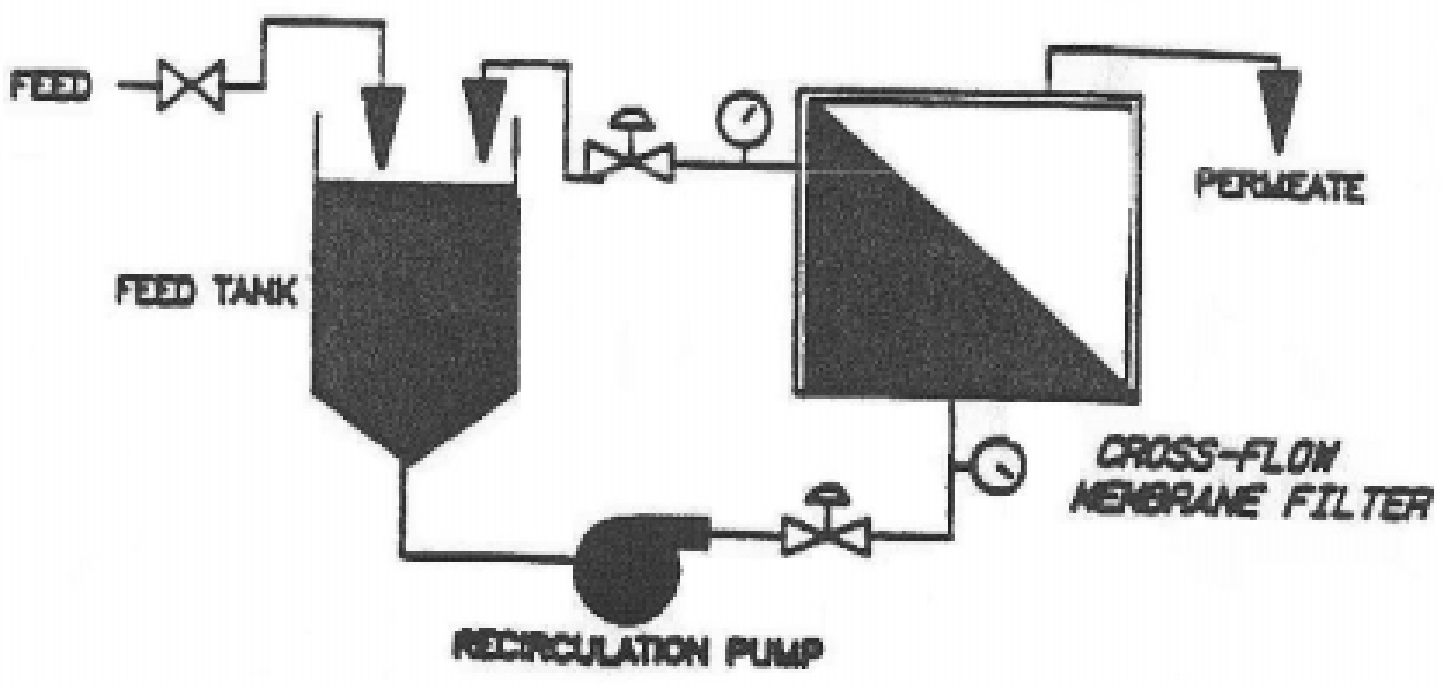

ВАТСН

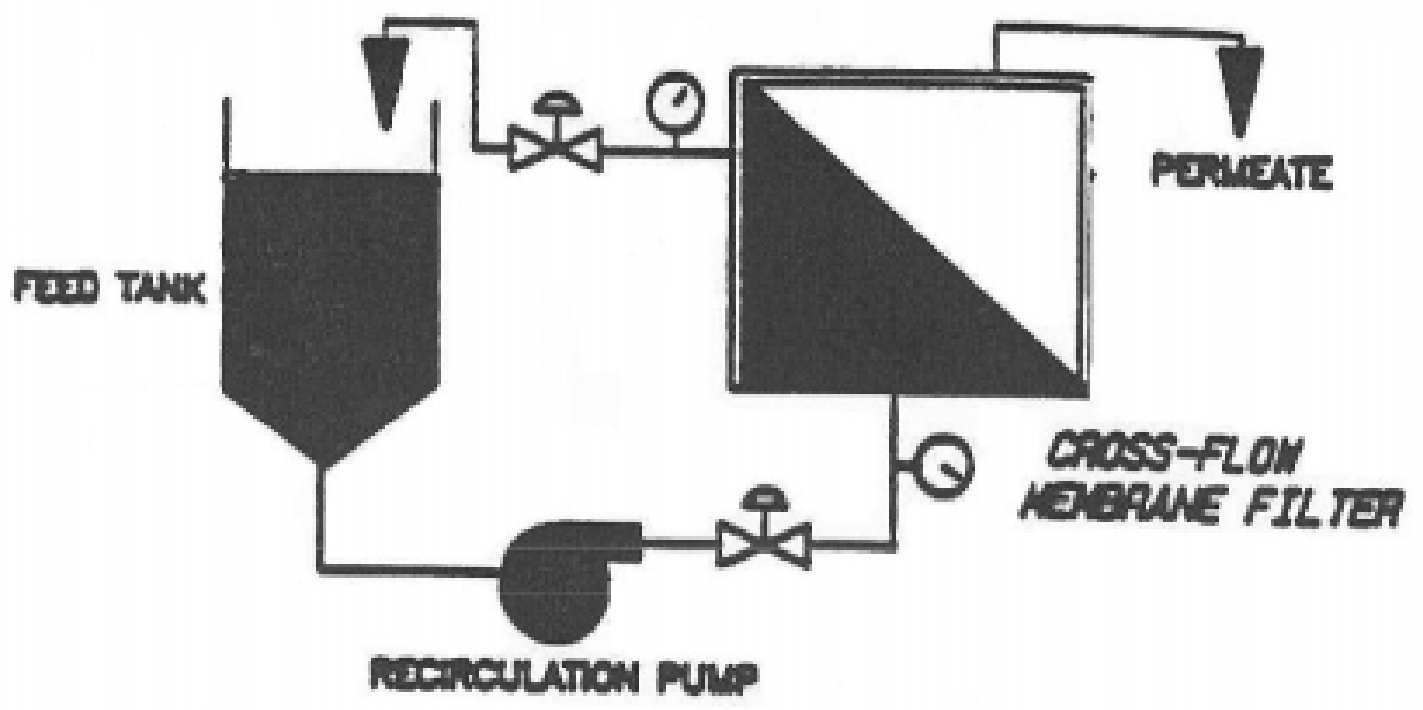

Figure (2.2)—Schematic Of Semi-Barch And Batch Operational Modes. 
batch operation). Semi-batch operation is the most frequently used operational mode in industrial applications.

\section{Batch Mode}

A schematic of batch operation mode was presented previously in Figure (2.2). In batch mode, which typically occurs at the end of semi-batch operation, fresh $1 \mathrm{X}$ solution is not added to the feed tank while permeate is removed. Thus, the feed volume is reduced and the concentrate remaining at the end of semi-batch operation is concentrated further. The concentration factor during batch operation is given in Equation (2.2).

$$
\mathrm{CF}_{\mathrm{b}}=\mathrm{CF}_{\mathrm{sb}} \times\left[\mathrm{V}_{\text {feed }} /\left(\mathrm{V}_{\text {feed }}-\mathrm{V}_{\text {perm }}\right)\right]
$$

where $\mathrm{CF}_{\mathrm{b}}=$ concentration factor during batch operation.

\section{General Types of Membrane Separation Processes}

Membrane processes are differentiated by the size of particles they separate. The four principle membrane separation techniques used to treat industrial waste and process waters are: reverse osmosis (RO), nanofiltration (NF), ultrafiltration (UF), and microfiltration (MF). The useful range of the four general membrane separation processes is presented in Figure (2.3) (after Cheryan (1986) and Perry and Green (1997)).

\section{Reverse Osmosis and Nanofiltration}

Membranes are characterized by the diameter of a particle or molecular weight of a molecule that is retained (known as the molecular weight cutoff (MWCO)) by the membrane. Additional details regarding membrane characteristics are presented later in Chapter 2.0. Reverse osmosis (RO) is a membrane separation technique used to separate 


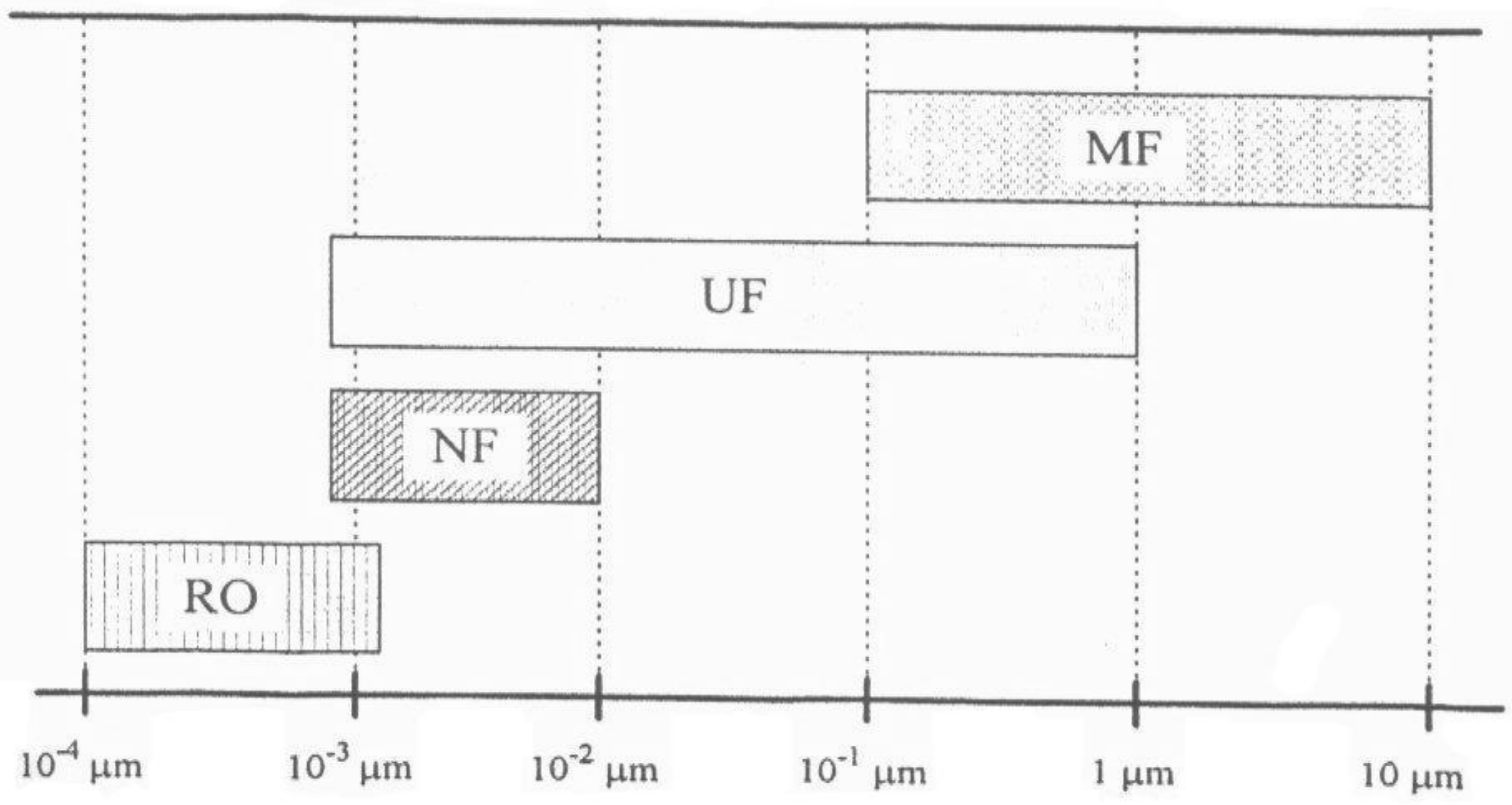

\section{Particle Diameter}

Figure (2.3)—The Useful Ranbes OF The Four General Membrane Separation Processes (After Cheryan (1986) And Perry And Green (1997)). 
materials with diameters from $\sim 10^{-4}$ to $10^{-3} \mu \mathrm{m}$ (less than $\sim 200$ to $2,000 \mathrm{MWCO}$ ). RO is generally used to remove salts and ions from solutions. The phenomena of osmosis and reverse osmosis are presented schematically in Figure (2.4). Osmosis is the flow of solvent (usually water) through a semi-permeable membrane from a region of low chemical potential to a region of higher chemical potential. For example, when a salt solution and pure water are separated by a semi-permeable membrane, a net flow from the pure water to the saline side of the membrane will occur due to differences in the chemical potential (and thus, the osmotic pressure) of the two solutions. The salt solution will rise to a point at which the head is equal to the osmotic pressure, as presented schematically in Figure (2.4). In RO, the pressure on the salt solution is raised above the osmotic pressure so the net movement of water is from the more concentrated saline side to the pure water side of the semi-permeable membrane. Transmembrane pressures ranging from 500 to 2,000 psi are common in RO applications.

Reverse osmosis has been used in the production of potable water, most prominently in the Middle East and on islands where the demand for potable water exceeds the natural supply. Small RO units have also been incorporated into multistage flash distillation systems to provide potable water sources on ocean vessels. Additionally, RO is used to create ultra high quality water in the manufacture of microelectronic circuits and in the dewatering and concentrating of beverage streams (e.g., dewatering orange juice prior to freezing). 

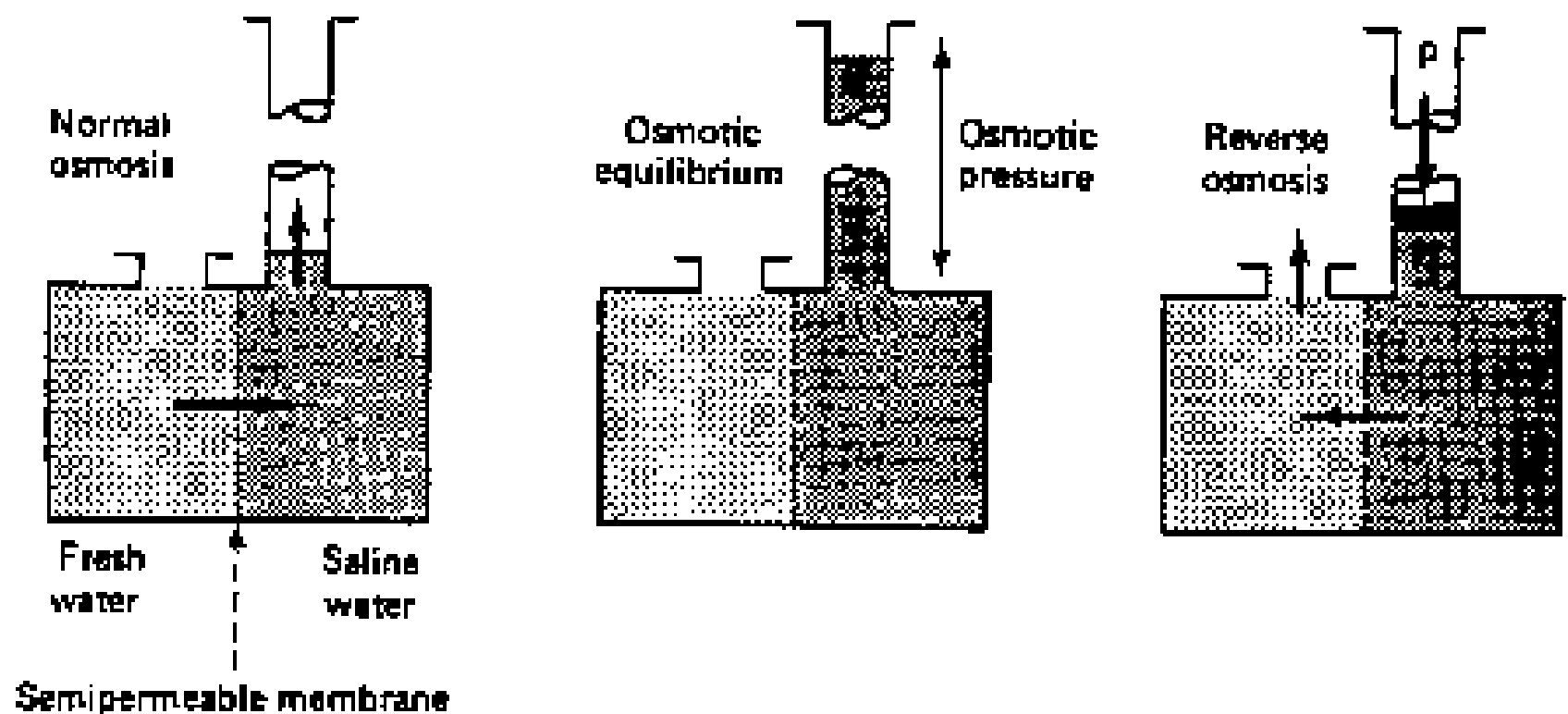

Figure (2.4)—The Phenomena of Osmosis and Reverse Osmosis. 
Nanofiltration (NF) systems operate under the same basic principle as reverse osmosis. However, nanofiltration is generally used to remove particles in the $\sim 10^{-3}$ to $10^{-2} \mu \mathrm{m}$ range ( 2,000 to 20,000 MWCO). Nanofiltration is often referred to as "loose RO" because nanofiltration operates on the same basic principle as RO but is applied to larger particle sizes. Smaller constituents of a waste or process stream (e.g., metal ions and dissolved salts) which would typically be removed using RO may pass through a nanofiltration membrane. Nanofiltration is often used to dewater pesticide and herbicide solutions. [Perry and Green 1997]

\section{Ultrafiltration and Microfiltration}

Ultrafiltration (UF) is a pressure-driven membrane technique based on a "sieving mechanism" in which particles are separated from solution based on size. UF is used to separate material in the $\sim 10^{-3}$ to $1 \mu \mathrm{m}$ size range $(2,000$ to $500,000 \mathrm{MWCO})$. Microfiltration (MF) is a similar membrane separation technique which is used to separate particles in the $\sim 10^{-1}$ to $10 \mu \mathrm{m}$ size range $(\sim 100,000$ to $>500,000 \mathrm{MWCO})$. Similarly to the case of reverse osmosis and nanofiltration, there is an overlap in the size range over which UF and MF are generally applied [Perry and Green 1997]. Thus, ultra and microfiltration are discussed as primarily one technique with the main distinction being the larger membrane pore size used in MF. Transmembrane pressures ranging from 5 to 150 psi are common in UF and MF applications.

Ultrafiltration has been successfully applied to the treatment of concentrated oily wastes from metal-working processes such as sheet rolling and die cutting. UF has been 
used to treat wastewaters high in oil, grease, and solids while reducing the volume of waste sent for disposal, while at the same time recovering alkaline process cleaners for reuse. Additionally, Cheryan (1986) summarized the results from several studies in which UF was demonstrated to be an effective treatment method for a wide variety of waste and process streams including: (1) the dairy industry for skim, whole milk, and cottage cheese whey processing; (2) biotechnology/bioengineering for protein fractionation and recovery of valuable microbes; (3) food processing for protein and juice extraction; and (4) for the recovery of ink in the printing industry. [Cheryan 1986, Perry and Green 1997]

Similarly to UF, microfiltration has been applied to the treatment of oily wastewaters. MF is used in water treatment to remove cryptosporidium and giardia cysts. Additionally, MF is frequently used in the dairy industry (1) as a non thermal method to remove bacteria from milk and protein products, (2) to separate fat from milk or dairy streams, and (3) to recover caustic agents used to clean dairy evaporators. [Perry and Green 1997]

\section{Configurations Of Conventional Membrane Modules}

The most common module configurations used in commercial cross-flow filtration are tubular, hollow fiber, spiral wound, and plate and frame [Belfort 1988].

\section{Tubular Membrane Modules}

A schematic of a tubular membrane module is presented in Figure (2.5) [MacNeil and McCoy 1989]. A tubular membrane consists of a porous membrane material cast on a permeable support matrix housed inside a rigid hollow tube. 


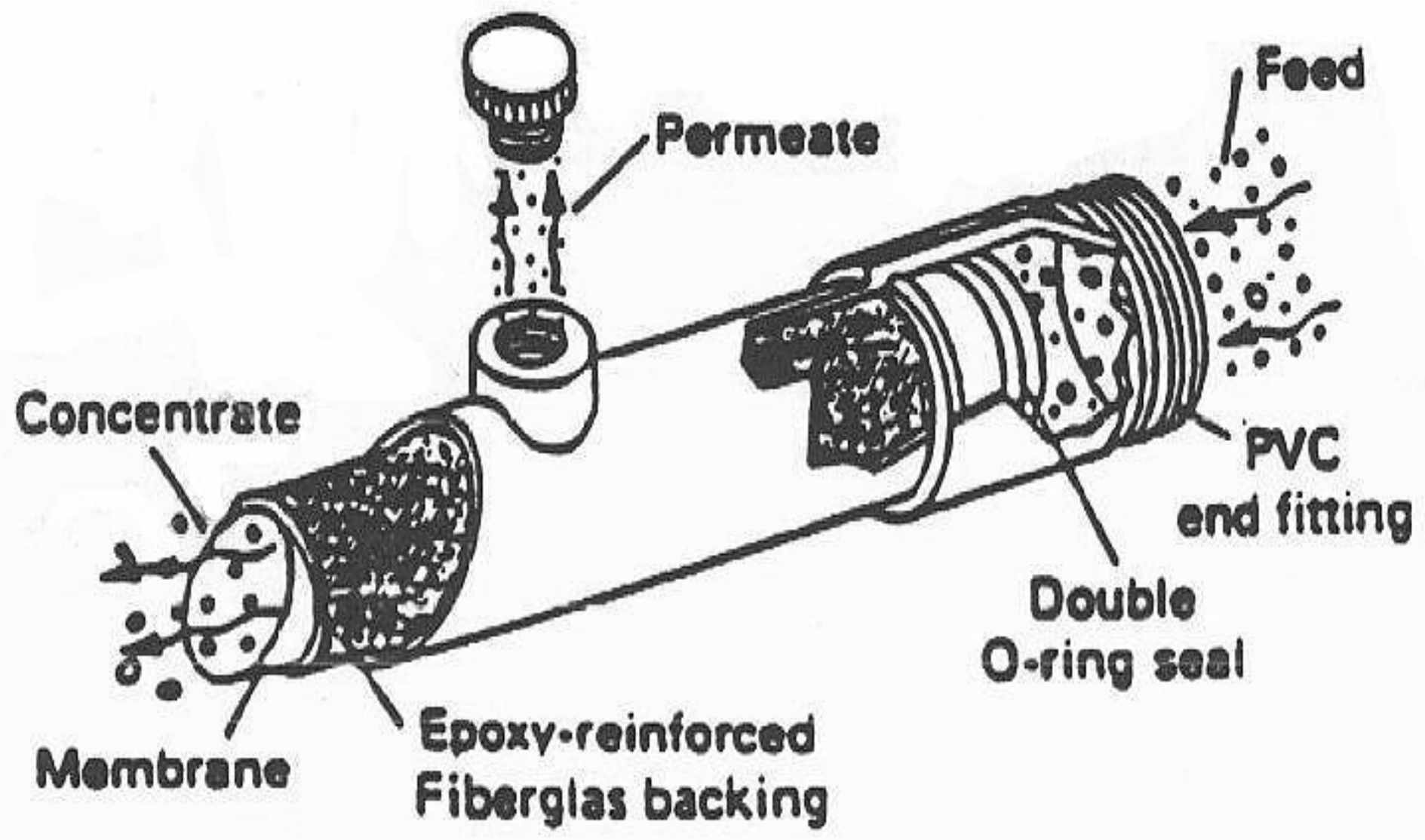

Figure (2.5)—Schematic Of A Tubular Membrane Module. 
Permeate is forced radially through the membrane under pressure and is collected through a port in the rigid outer casing [Belfort 1988]. Tubular membranes are available in single or multi-tube configurations. Typical commercial tubular membranes can be operated at pressures up to $90 \mathrm{psig}$. These modules are generally 10 feet long and have inner tube diameters ranging from 0.5 to 1 inch. Typical flow rates necessary to achieve a sufficient shear in a 1 inch tubular membrane range from $\sim 30$ to $40 \mathrm{gal} / \mathrm{min}(\sim 114$ to $151 \mathrm{~L} / \mathrm{min}$.) Due to large channel sizes (typically 0.5 to 1 inch), tubular membranes are commonly used to treat waste and process streams with high solids concentrations. Additionally, the large flow channels make it easier to remove accumulated solute molecules using mechanical and chemical cleaning techniques [MacNeil and McCoy 1989]. The disadvantages of these membrane modules include a relatively low permeate flow rate per unit volume and a high volume hold-up per unit area of membrane [Belfort 1988].

\section{Hollow Fiber Membrane Modules}

A schematic of a hollow fiber membrane module is presented in Figure (2.6) [MacNeil and McCoy 1989]. Hollow fiber membrane modules consists of a bundle of fine tubes (typical inside diameters range from $\sim 20$ to $106 \mathrm{~mm}$ ) made of the membrane material bound at each end of the module by epoxy discs. Unlike tubular membranes, hollow fiber membranes do not have an underlying support structure. Feed is forced into the membrane module radially under pressure through a perforated tube in the center of the housing. Permeate flows through the individual fibers and is collected through epoxy tube sheets at the end of the module [MacNeil and McCoy 1989]. Transmembrane pressures of up to $40 \mathrm{psig}$ are common in hollow fiber modules [Belfort 1988]. 


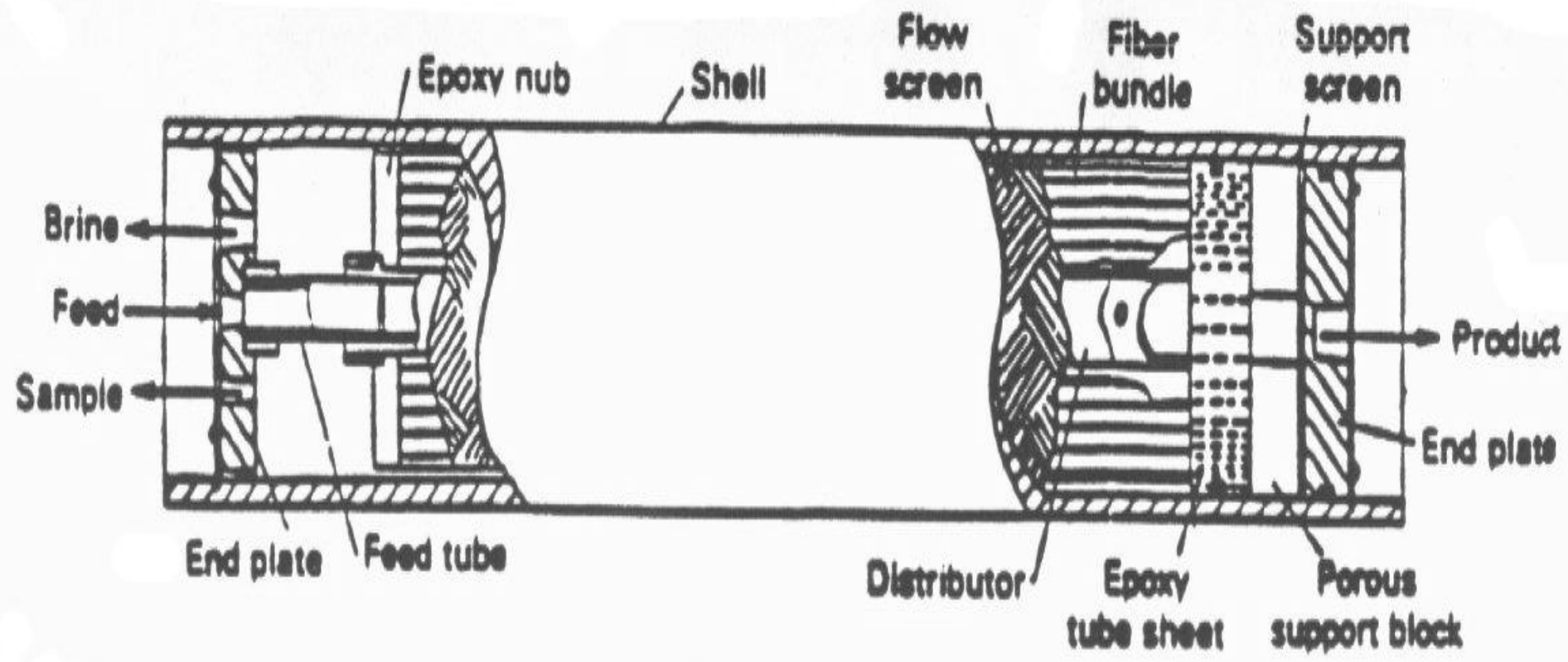

Figure (2.6)—Schematic Of A Hollow Fiber Membrane Module. 
Additionally, hollow fiber membrane modules have a higher packing density than tubular modules but are more subject to fouling effects than tubular systems. Hollow fiber membranes cannot be mechanically cleaned [MacNeil and McCoy 1989]. However, the direction of permeate flow can be changed to effectively back-flush the membrane to remove fouling materials from the surface. Typically, lower final waste concentrations are obtained using a hollow fiber system than would be achieved with a tubular module due to pore plugging/fouling as the feed concentration is increased. [Belfort 1988]

\section{Spiral Wound Membrane Modules}

A schematic of a spiral wound membrane module is presented in Figure (2.7). A spiral wound membrane module consists of alternating layers of porous membranes, permeate carriers, and spacers, wrapped around a central hollow permeate collection tube. The feed solution flows axially through the wound membrane module. Permeate is forced through the porous membrane and is collected through the hollow center tube while rejected feed solution exits at the end of the module. Spiral wound membrane modules range from $\sim 5$ to $20 \mathrm{~cm}$ in diameter and can be operated at pressures up to $\sim 150$ psig. Typically, spiral wound membranes foul more easily than tubular membranes due to smaller waste flow channels (common spacer thicknesses range from 28 to $80 \mathrm{~mm}$ ). This factor, coupled with the fact that spiral wound modules cannot be mechanically cleaned limits the application of these membrane configurations to waste and process streams with low solids concentrations [MacNeil and McCoy 1989]. However, spiral wound membranes have a significantly higher packing density than other configurations, 


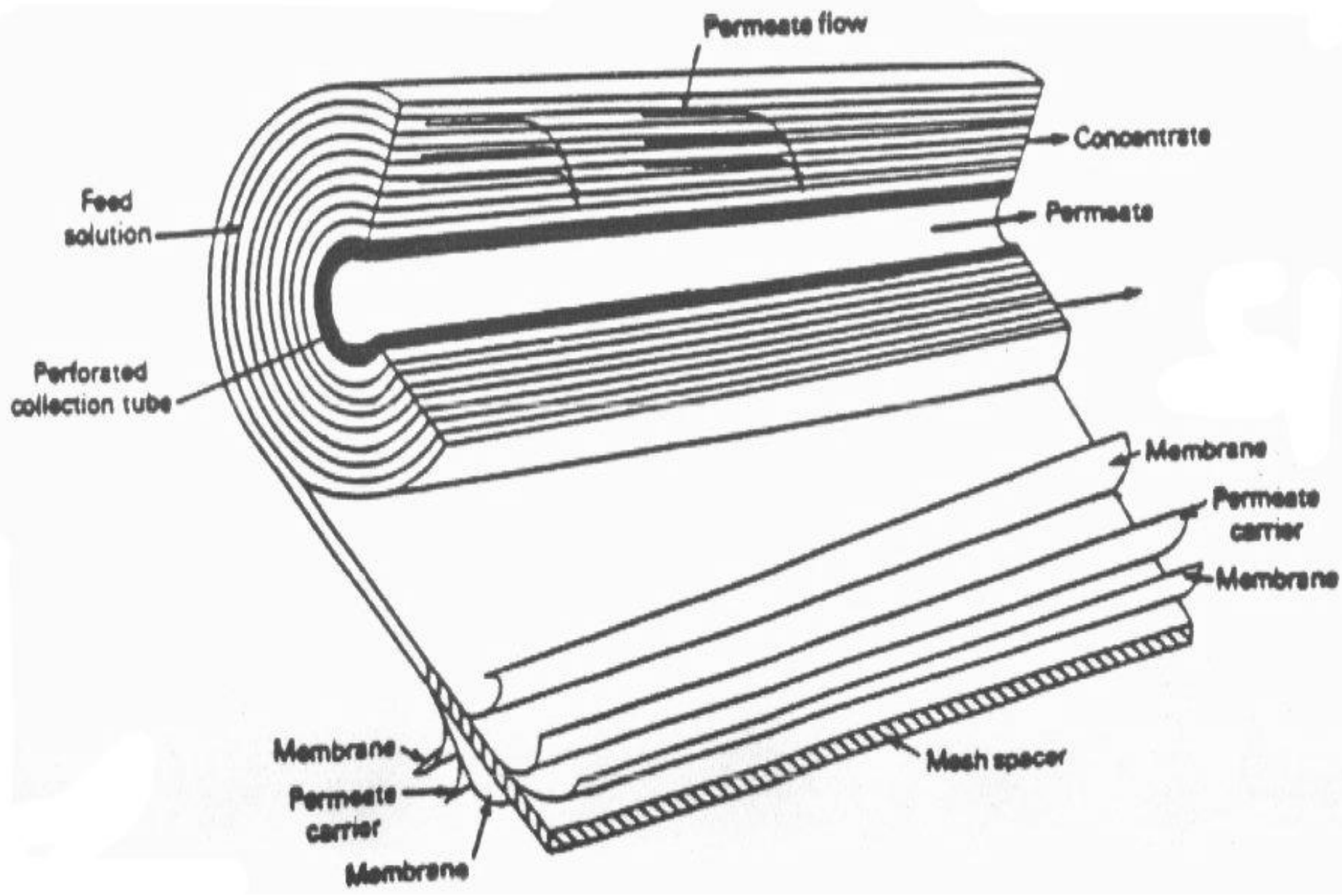

Figure (2.7)—Schematic Of A Spiral Wound Membrane Module. 
with high volume flow rates (and low solids concentrations) [Eringis et al. 1993]. Spiral wound units have primarily been used in laboratory-scale filtration units to recover proteins in the biotechnology field and have been considered for large-scale commercial bioprocessing applications [Belfort 1988].

\section{Plate and Frame Membrane Modules}

A schematic of a plate and frame membrane module is presented in Figure (2.8) [MacNeil and McCoy 1989]. In these systems, hollow disc-shaped spacers located between porous membranes are used to direct the feed radially along the membrane surface. A stack of multiple membranes separated by spacers is placed in series or in parallel inside a cylindrical housing. Permeate is forced through the membrane under pressure and is collected at the outer edges of the membrane disc plate. Concentrated solute is collected at the top of the stack. The advantages of this configuration include a relatively low volume hold-up per unit membrane area (a feature which is attractive for the recovery of valuable biologicals), and a thin channel height (typically 0.3 to $0.6 \mathrm{~mm}$ ) which permits these units to process highly viscous solutions. The disadvantages of this membrane configuration include susceptibility to channel plugging and difficulties in mechanical cleaning [Belfort 1988]. Additionally, the commercial application of plate and frame membrane configurations has been limited due to high capital cost relative to other filtration units [MacNeil and McCoy 1989]. 


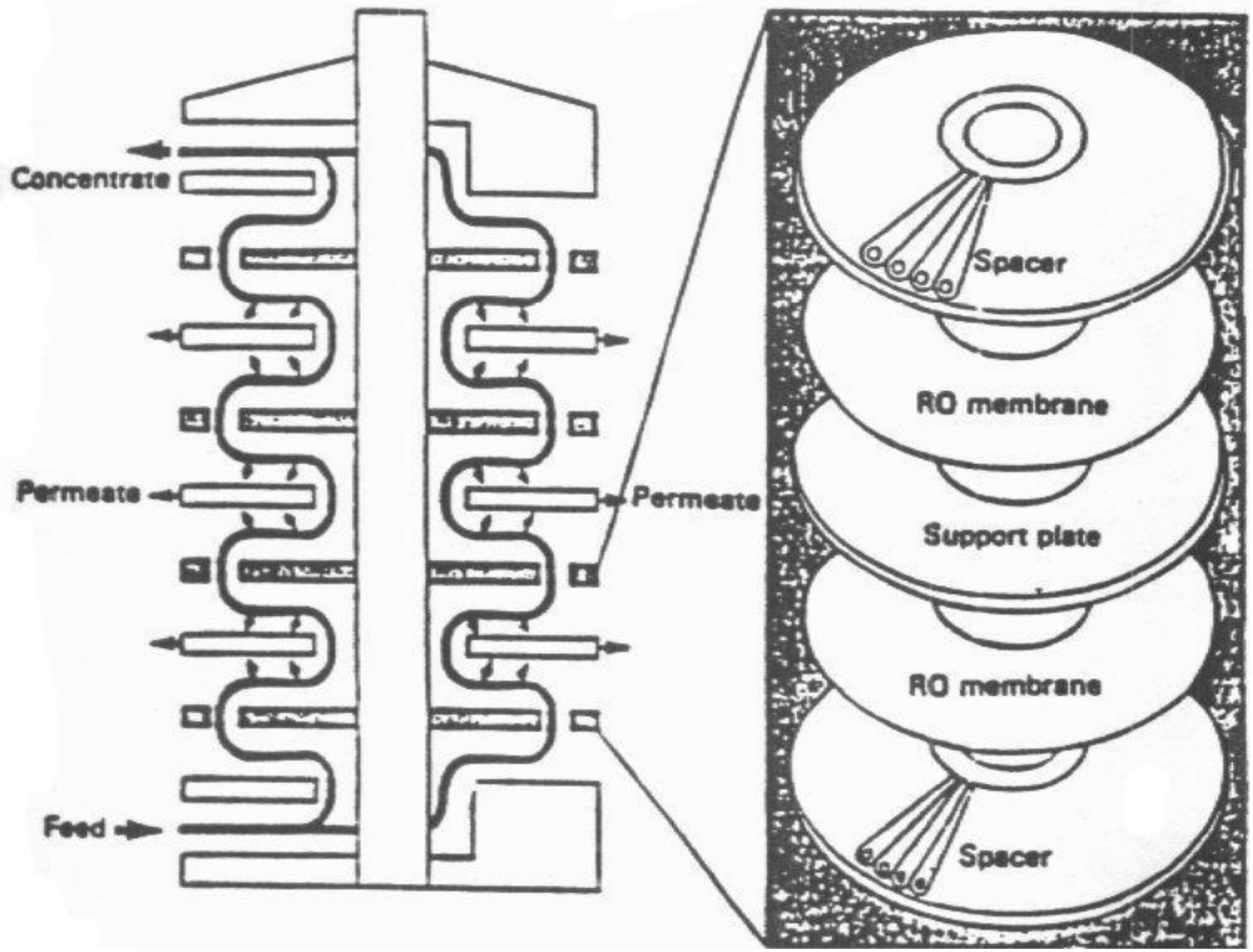

Figure (2.8)—Schematic Of A Plate And Frame Membrane Module. 


\section{Membranes}

Membranes used in membrane separation processes are characterized by the diameter of a particle or molecular weight of a molecule that is retained (i.e., not allowed to pass through) by the membrane. In theory, compounds having a molecular weight greater than the molecular weight cut off (MWCO) will be retained by the membrane and compounds with molecular weights less than the MWCO will pass through the membrane as permeate. However, the MWCO designation is somewhat misleading because a molecule having a molecular weight less than the membrane MWCO may still be retained by the membrane because of its unique three dimensional geometry.

Membrane materials must be able to withstand the mechanical, chemical, and biological stresses imposed during operation and cleaning without suffering appreciable losses in operating efficiency. Mir et al. (1977) discussed several general operational and feed parameters which must be considered in the treatment of wastewaters. The factors include $\mathrm{pH}$, ionic strength, temperature, and pressure. Additionally, membrane surface properties can also affect permeate flux and quality. Electrostatic repulsion between the membrane and the contaminant can be used to enhance waste solute retention and increase flux.

Membrane resistance is a measure of the hydraulic resistance to flow through a pore channel and is an intrinsic material property of the membrane due to the material properties of the membrane. The membrane resistance can be determined by applying Darcy's Law for a virgin membrane with a clean water feed: 


$$
\mathrm{J}=\mathrm{P}_{\mathrm{tmp}} / \mu \mathrm{R}_{\mathrm{m}}
$$

where, $\mathrm{J}=$ permeate flux, $\mathrm{P}_{\mathrm{tmp}}=$ transmembrane pressure, $\mu=$ water viscosity (absolute), and $\mathrm{R}_{\mathrm{m}}=$ membrane resistance. The assumption of well developed laminar flow through the membrane pore channel is made when using Equation (2.3). The relationship between permeate flux and transmembrane pressure for a clean water feed is presented in Figure (2.9). When clean water is used as the feed, the slope of a regression line through the origin on the plot of permeate flux versus transmembrane pressure is inversely proportional to the product of the feed viscosity and the resistance of the membrane, $R_{m}$, as presented in Figure (2.9) [Cheryan 1986]. In this case, the membrane resistance is:

$$
\mathrm{R}_{\mathrm{m}}=1 /\left(\mu^{*} \text { slope }\right)
$$

A summary of typical resistances for several ceramic (aluminum oxide) micro and ultrafiltration membranes studied by Nazzal and Wiesner (1996) is presented in Table (2.1). Additionally, a resistance of $\sim 10^{12} \mathrm{~m}^{-1}$ was measured by Lipp et al. (1988) for a polymeric membrane used to treat oil-in-water emulsions in a stirred cell UF unit (membrane material and MWCO were not specified). The MF and UF membrane resistances reported by Nazzal and Wiesner (1996) and Lipp et al. (1988) were comparable to values measured by other researchers [Gutman 1987, Bhave 1991].

Nazzal and Wiesner (1996) have developed a model for calculating 


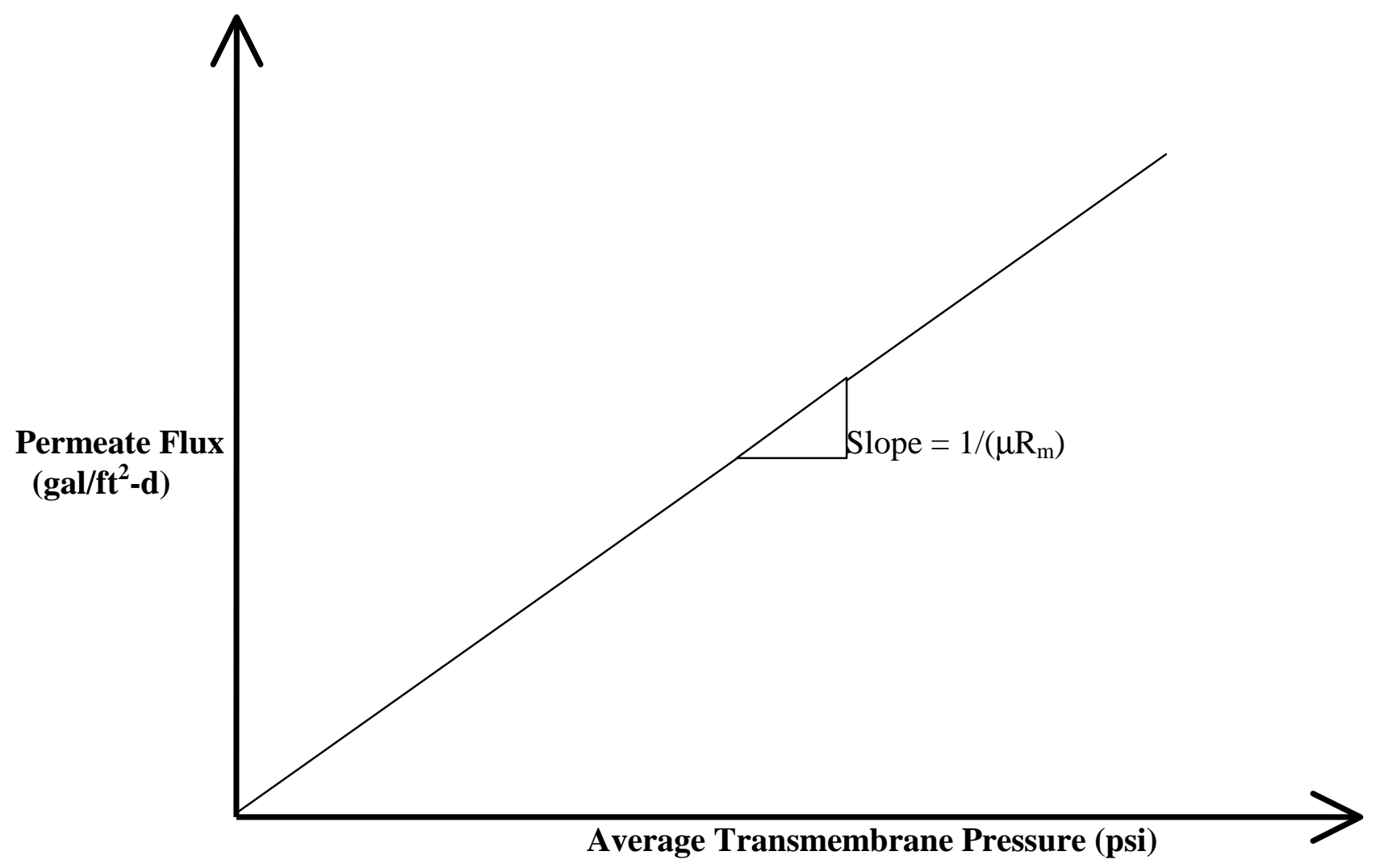

Figure (2.9)—The Relationship Between Permeate Flux And Transmembrane Pressure For A Clean Water Feed. 
Table (2.1)--Typical Membrane Resistances For Micro And Ultrafiltration Membranes [Nazzal And Wiesner 1996].

\begin{tabular}{|c|c|}
\hline \hline Pore diameter $(\boldsymbol{\mu m})$ & Membrane resistance $\left(\mathbf{m}^{\mathbf{- 1}}\right)$ \\
\hline \hline 0.8 & $1.5 \times 10^{11}$ \\
\hline 0.5 & $2.0 \times 10^{11}$ \\
\hline 0.2 & $5.0 \times 10^{11}$ \\
\hline 0.05 & $6.0 \times 10^{11}$ \\
\hline
\end{tabular}


the critical pressure, $\mathrm{P}_{\text {crit }}$, required to force the entry of an oil droplet into a membrane pore based on the Young-Laplace equation. A schematic of the passage of an oil droplet through the pores of a membrane is presented in Figure (2.10). The critical pressure is given by:

$$
\mathrm{P}_{\text {crit }}=\left(2 \gamma_{\mathrm{o} / \mathrm{w}}\right)\left(1 / \mathrm{r}^{*}-1 / \mathrm{R}^{*}\right)
$$

where $\gamma_{\mathrm{o} / \mathrm{w}}=$ the interfacial tension between the oil and water, $\mathrm{r}^{*}=$ the radius of curvature of the advancing portion of the drop, and $\mathrm{R}^{*}=$ the radius of curvature of the lagging drop interface. This model presented in Equation (2.5) can be further expressed as:

$$
\begin{array}{r}
\mathrm{P}_{\text {crit }}=\left(2 \gamma_{\mathrm{o} / \mathrm{w}} \cos \theta / \mathrm{r}_{\text {pore }}\right)\left\{1-\left[\left(2+3 \cos \theta-\cos ^{3} \theta\right) /\right.\right. \\
\left.\left.\left[4\left(\mathrm{r}_{\text {drop }} / \mathrm{r}_{\text {pore }}\right)^{3} \cos ^{3} \theta-\left(2-\sin \theta+\sin ^{3} \theta\right)\right]\right]^{1 / 3}\right\}
\end{array}
$$

where $\theta=$ contact angle, $r_{\text {drop }}=$ radius of the oil drop, and $r_{\text {pore }}=$ radius of the membrane pore. Equation (2.6) predicts that a membrane with a smaller pore size should require a higher transmembrane pressure to initiate oil droplet movement into the membrane pores for a given interfacial tension, contact angle, and drop size. Nazzal and Wiesner cited a common value for the contact angle, $\theta$, to be $155^{\circ}$ and a typical interfacial tension for oilin-water emulsions, $\gamma_{\mathrm{o} / \mathrm{w}}$, to be 55 dynes $/ \mathrm{cm}$.

Contrary to the theoretical expectation of a distinct drop in solute rejection at or near $\mathrm{P}_{\text {crit }}$, distinct cutoffs in oil rejection were not observed at the critical pressure. Nazzal and Wiesner attributed this unexpected behavior to variations in the pore size around the nominal pore diameter in 


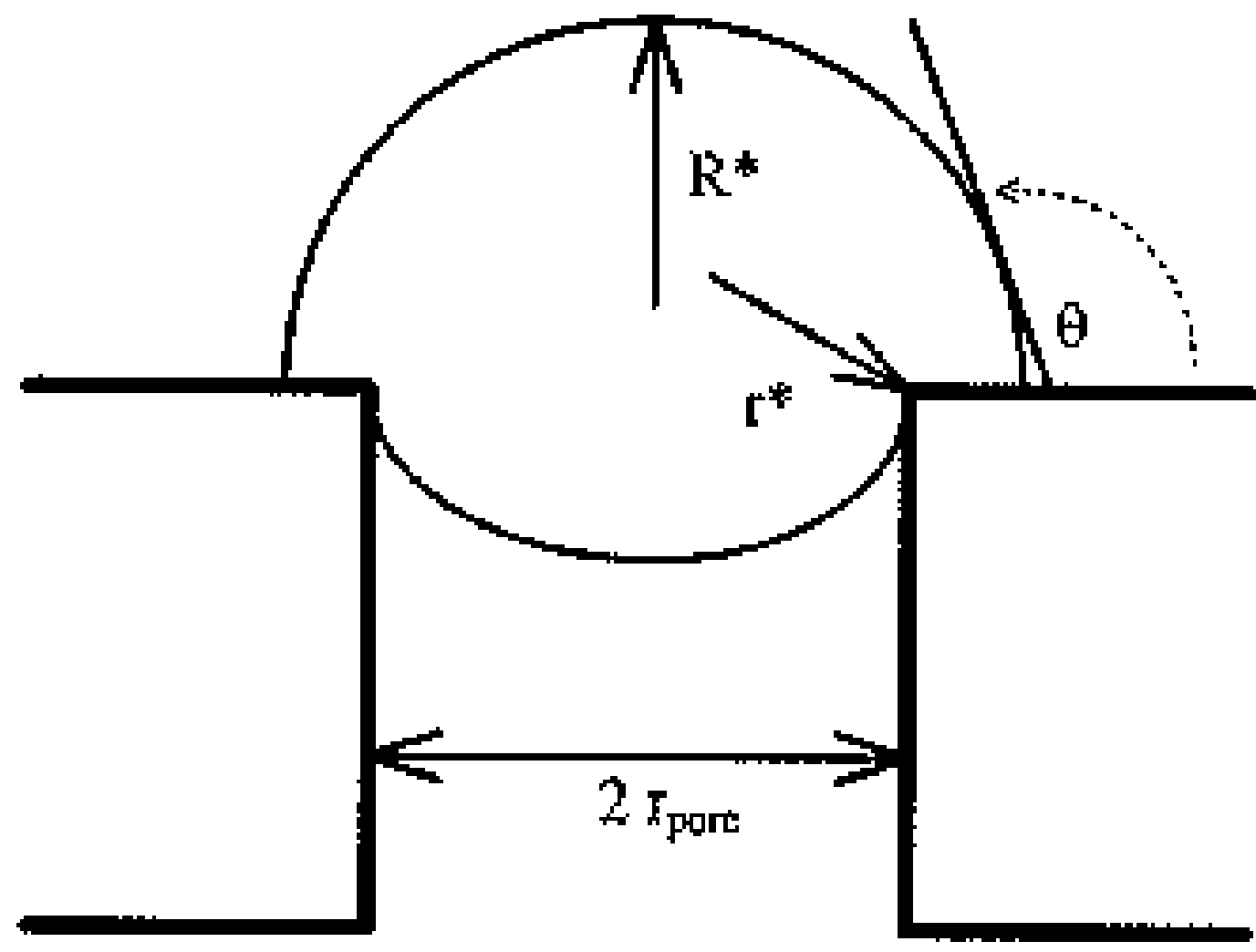

Figure (2.10)—Schematic Of The Passage Of An Oil Droplet Through The Pores Of A Membrane. 
addition to the polydispersivity of oil droplet sizes (as observed by Lee et al. (1988)). Due to these factors, Nazzal and Wiesner recommend using Equation (2.6) as a rough estimate of the critical pressure and suggested including allowances for membrane pore and oil drop size variation when applying this model in practice. Additionally, the authors noted that this model may be valid when the membrane cut-off and hydrodynamics favor the accumulation of oil near the membrane surface (due to a change in the contact angle as the membrane becomes more oil-wet).

In Equation (2.7), a model similar to Equation (2.6) was used by Lee et al. (1984) to predict the pressure necessary to force an oil droplet through the pores of a membrane.

$$
P_{\text {cap }}=\left(2 \gamma_{\mathrm{o} / \mathrm{w}}\right)[\cos (\theta) / \mathrm{r}]
$$

where $P_{\text {cap }}=$ capillary pressure of an emulsified oil droplet, $\gamma_{o / w}=$ the interfacial free energy (surface tension) between the oil and water, $\theta=$ contact angle, and $r=$ radius of the membrane pores. The capillary pressure of an emulsified oil drop is used as the basis for describing oil droplet rejection. Typically, the value of the contact angle, $\theta$, exceeds $90^{0}$ which implies that the membrane is more hydrophilic than oleophilic. For values of the contact angle in excess of $90^{\circ}$, the capillary pressure is negative and prevents the passage of oil droplets through the membrane pores against the applied pressure. Lee et al. assumed a contact angle of $180^{\circ}$. Lee et al. (1984) investigated the use of the capillary pressure model to predict the passage of oil through a polymeric membrane with a $20 \mathrm{k}$ MWCO in an Amicon S4OC stirred UF cell. In these experiments, initial oil concentrations of 1 to 5 vol.\% were used at a constant feed temperature of $20{ }^{0} \mathrm{C}$ and a 
constant stirring speed of $300 \mathrm{rpm}$. A breakthrough of oil droplets was not observed when the feed pressure was increased above the capillary pressure as calculated in Equation (2.7). The authors presented an explanation of this phenomena similar to that of Nazzal and Wiesner. Since the sizes of the membrane pores varied over a non-uniform distribution, the number of pores where the operating pressure exceeded the capillary pressure increased as the applied pressure was raised resulting in a more gradual breakthrough of oil than expected.

\section{Factors Controlling Permeate Flux}

\section{Concentration Polarization}

The theoretical relationship between permeate flux, transmembrane pressure, feed concentration, and hydraulic turbulence is presented in Figure (2.11). Under conditions of low transmembrane pressure, low feed concentration, and high hydraulic turbulence, permeate flux is linearly proportional to transmembrane pressure. The linear permeate flux/transmembrane pressure relationship is idealized in the case of a clean water feed. When a clean water feed is ultrafiltered there are theoretically no contaminants to either (1) plug the membrane pores or (2) accumulate on the membrane surface.

The buildup of solute at the membrane surface is known as "concentration polarization" and is largely responsible for the deviation of the product flux from the linear flux-pressure model presented in Equation (2.3). Primarily, three competing solute transport mechanisms occur in ultrafiltration: (1) convective transport of solute to the membrane surface (2) back diffusion of accumulated solute to the bulk solution, and (3) solute permeation through the membrane as presented schematically in Figure (2.12). 


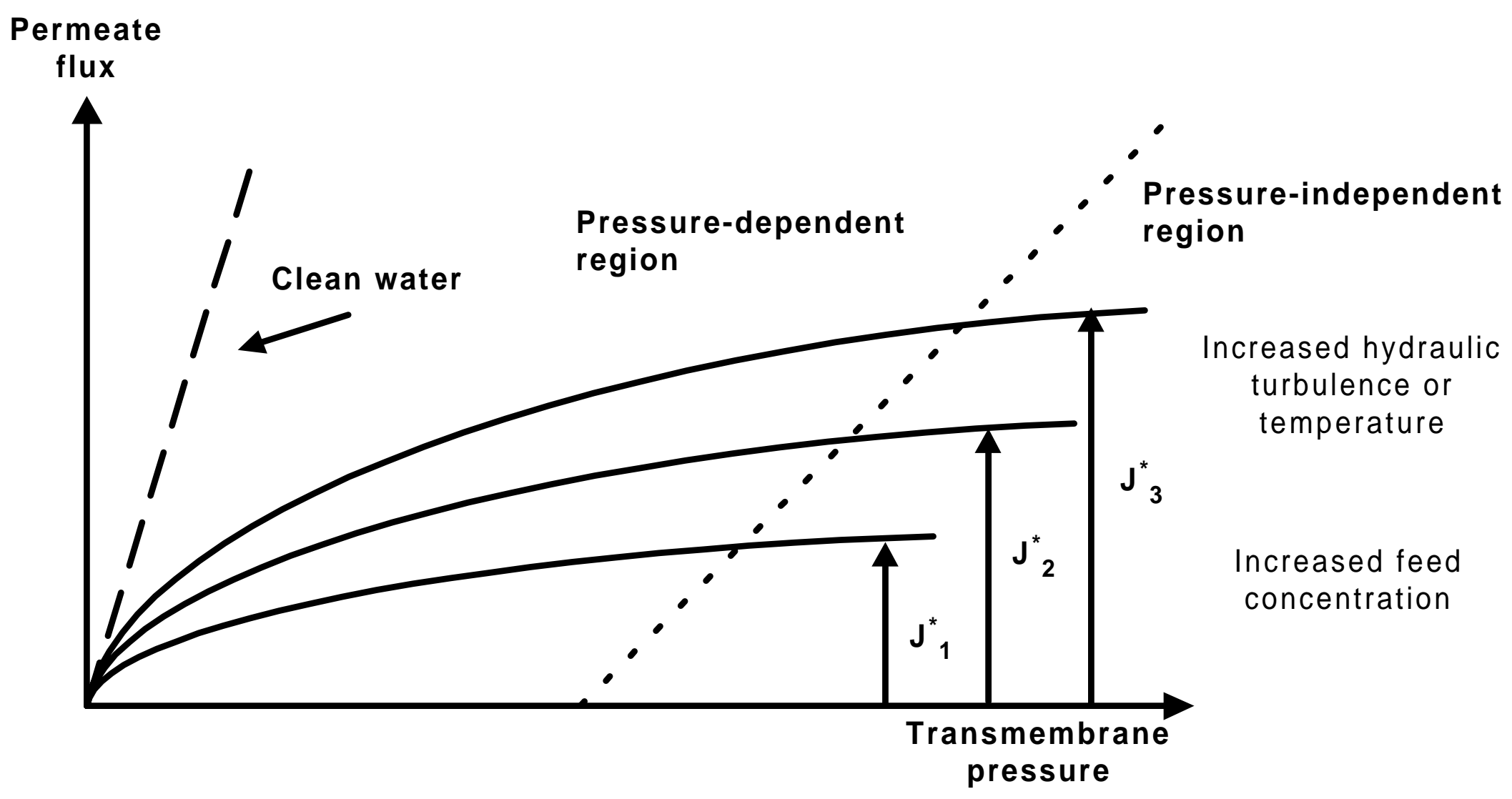

Figure (2.11) — The Theoretical Relationship Between Permeate Flux, Transmembrane Pressure, Feed Concentration, And Hydraulic Turbulence. 


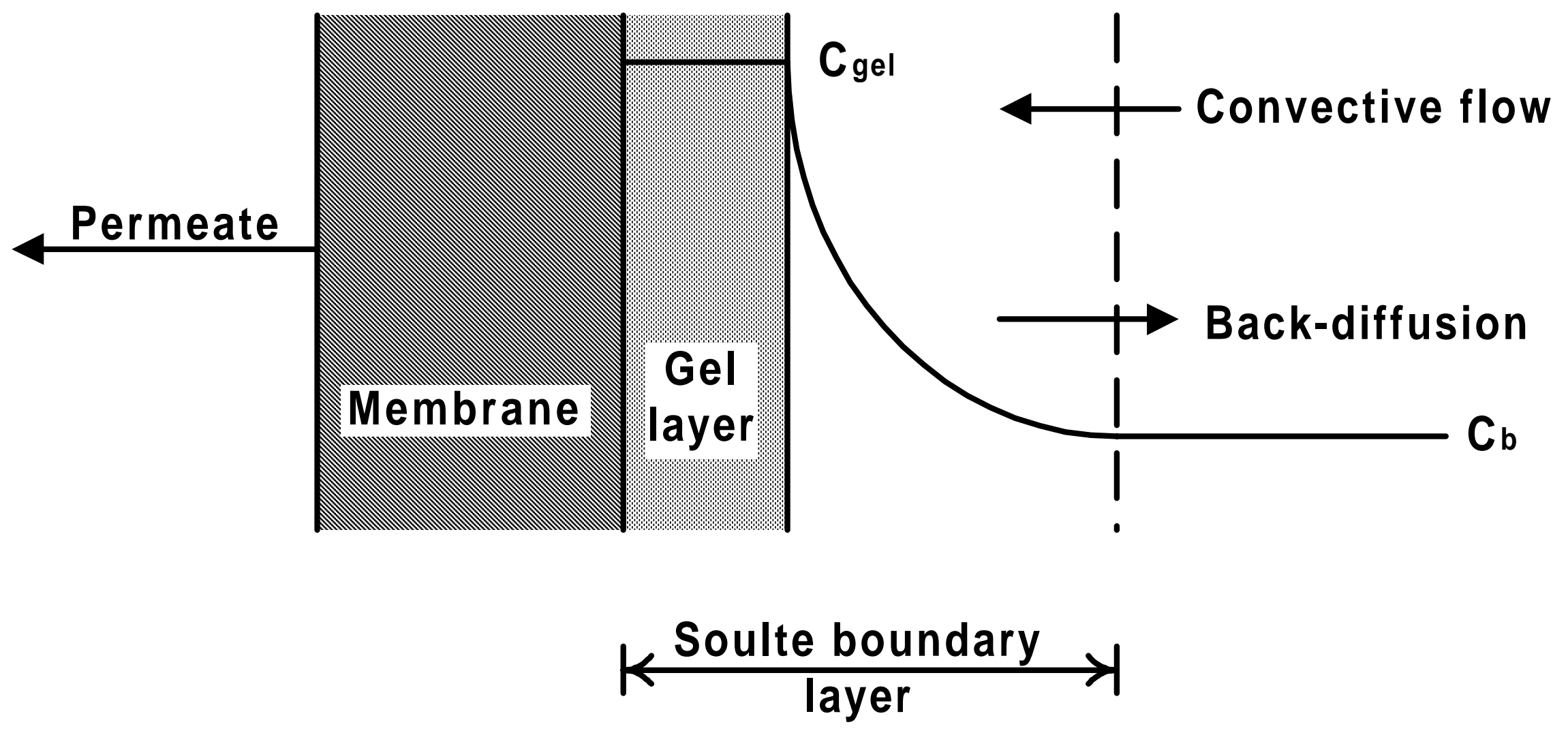

Figure (2.12)—Schematic Of The Three Primary Solute Transport Mechanisms In Membrane Separation Systems. 
Solute is convectively transported to the porous membrane surface and a fraction of the solvent is removed as permeate which results in a locally higher solute concentration at the membrane surface relative to the bulk solute concentration. As the transmembrane pressure is increased, feed concentration is increased, or hydraulic turbulence is decreased, the permeate flux becomes independent of pressure.

In general, there are four operational and feed solution parameters which contribute to the formation of the polarized solute concentration boundary layer and subsequent asymptotic permeate flux response to increased transmembrane pressure: (1) transmembrane pressure, (2) feed concentration, (3) hydraulic turbulence, and (4) feed viscosity/temperature. Transmembrane pressure is the principle driving force in membrane separation systems. However, as transmembrane pressure is increased, there is a corresponding increase in convective transport of solute molecules to the membrane surface. A thicker solute boundary layer forms as a result of the enhanced convective transport of solute molecules to the membrane surface and the permeate flux becomes independent of transmembrane pressure.

Solute concentration in the feed is another parameter which limits permeate flux. As the solute concentration in the feed increases, permeate flux decreases due to the accumulation of solute molecules at the membrane surface. At high solute feed concentrations, it becomes difficult to pump a feed solution at high enough to minimize the thickness of the polarized concentration boundary layer. Thus, the permeate flux decreases as the solute concentration increases. The hydraulic turbulence in membrane separation systems also plays an important role in maintaining a satisfactory permeate 
flux. As hydraulic turbulence is increased, the permeate flux should theoretically also increase due to a decrease in the thickness of the polarized concentration boundary layer. Similarly, as the temperature of the feed solution is increased, permeate flux increases due to: (1) a decrease in the viscosity of the feed solution (which makes it easier to pump the feed solution at a high cross-flow velocity) or (2) an increase in the solute diffusivity (which results in an increased diffusion of solute molecules from the membrane surface back to the bulk solution). [Cheryan 1986]

Cheryan (1977) noted three operational characteristics which can be used to judge the extent of concentration polarization in a UF system: (1) deviation from the pure water line, (2) relatively rapid attainment of pressure-independent flux behavior, and (3) marked hysteresis effect observed on lowering the pressure. Although the deviation from the pure water flux may be attributed to a decrease in the driving force due to increased osmotic pressure, Cheryan believed the primary mechanism of flux decline was the increased $\mathrm{h}$ hydrodynamic resistance in the concentration boundary layer. Cheryan supported the argument in favor of flux decline due to the formation of a polarized gel layer based on prior knowledge of the gel characteristics of soybean proteins and the gel-like behavior of highly structured macromolecules (at concentrations in excess of $\sim 10 \mathrm{wt}$. \%) which were in good agreement with experimental values. Additionally, the hysteresis effects observed as pressure was varied in these experiments supported the hypothesis that interactions had occurred between solute molecules which were approaching a consolidated "close packed" configuration. Citing the marked differences in the mass transfer properties of feed solutions, Cheryan suggested that mass transfer models will 
have to be supplemented with experimental data to be used in UF process design [Cheryan 1977, Cheryan 1986].

\section{Membrane Fouling}

Membrane fouling can also result in a decrease in permeate flux over time. Fouling is a term used to describe the loss of throughput of a membrane device as it becomes chemically or physically changed by a process fluid. Fouling is different from concentration polarization. Both phenomena result in a reduced membrane system output and the resulting resistances due to both phenomena are additive. For example, when the flow rate in a cross-flow membrane separation system is reduced, permeate flux will decrease. If the decrease in permeate flux is due only to the effects of concentration polarization (i.e., the accumulation of solute molecules at the membrane surface), the permeate flux should return to the initial value when the flow rate is raised. However, if the permeate flux does not increase to the initial value when the flow rate is increased, membrane fouling is the operative flux decline phenomena.

Membrane fouling is primarily due to (1) pore plugging and/or (2) solute adsorption. In the case of pore plugging, solute molecules or other minor constituents of a feed stream (e.g., bacteria) are forced into the membrane pores resulting in a loss of permeate flow channels and thus a decrease in permeate flux. Pore plugging becomes more prevalent under operating conditions such as: low hydraulic turbulence and high transmembrane pressure. At low hydraulic turbulence, a thicker solute layer accumulates on the membrane surface. When combined with high transmembrane pressure, the potential for forcing solute molecules into the membrane pores increases. Additionally, if 
solute molecules are small enough to enter the membrane pores, some solute may be adsorbed onto the pore walls. The adsorbed molecules decrease the effective pore diameter. As the effective pore diameter is decreased, the overall resistance to hydraulic flow increases and a decreased in permeate flux is observed. Solute adsorption is due principally to physical and/or chemical properties of the feed solution. [Jonsson and Tragardh 1990]

In both cases, the proper selection of operating conditions, feed pretreatment, and cleaning methods are important aspects to minimizing flux loss due to membrane fouling. For example, the transmembrane pressure should be balanced to provide maximum permeate flux at the minimum transmembrane pressure. Thus, an understanding of the flux-pressure relationship presented schematically in Figure (2.11) is important to the minimization of membrane fouling. Specifically, a membrane separation system should not be run at transmembrane pressures in excess of the value necessary to achieve a masstransfer-controlled permeate flux.

Feed pretreatment is often stream or site-specific and has received little attention in the literature. However, steps such as removing free oil from a waste or process stream prior to the application of membrane separation are often used to minimize potential membrane fouling.

The primary method of restoring permeate flux lost to membrane fouling is membrane cleaning. Fouling which can be removed through membrane cleaning is called "reversible fouling" and fouling which cannot be removed through cleaning is called "irreversible fouling." Cleaning procedures are highly stream-specific. Although 
cleaning is used to restore permeate flux, the use of aggressive chemicals such as concentrated acidic and caustic solutions often results in shortened membrane life due to affects on membrane surface properties. [Perry and Green 1997]

\section{Factors Controlling Permeate Water Quality}

Size and Shape of Solute Molecules

Solute rejection in a membrane separation process is heavily influenced by membrane pore size. In general, molecules larger than the membrane pore size are retained while smaller molecules pass through as permeate. Therefore, permeate water quality is affected by the membrane pore size as well as the size of solute molecules in the feed solution. The shape of individual solute molecules in the feed solution also affects permeate water quality. A schematic of solute molecules at the membrane surface and permeating through the membrane pores is presented in Figure (2.13). Assuming the three solute molecules presented in Figure (2.13) have identical molecular weights, it can be observed that solute permeation through the membrane depends on the shape of each individual molecule. Regardless of molecular weight, the shape of a solute molecule can affect the rejection of that molecule by the membrane [Cheryan 1986].

Membrane Material and Configuration

Permeate water quality can also be affected by membrane material or configuration. Two membranes made of different materials, having identical molecular weight cutoffs, may have different solute rejections. These differences can be attributed to varying pore size distribution or chemical interactions between membrane materials and 


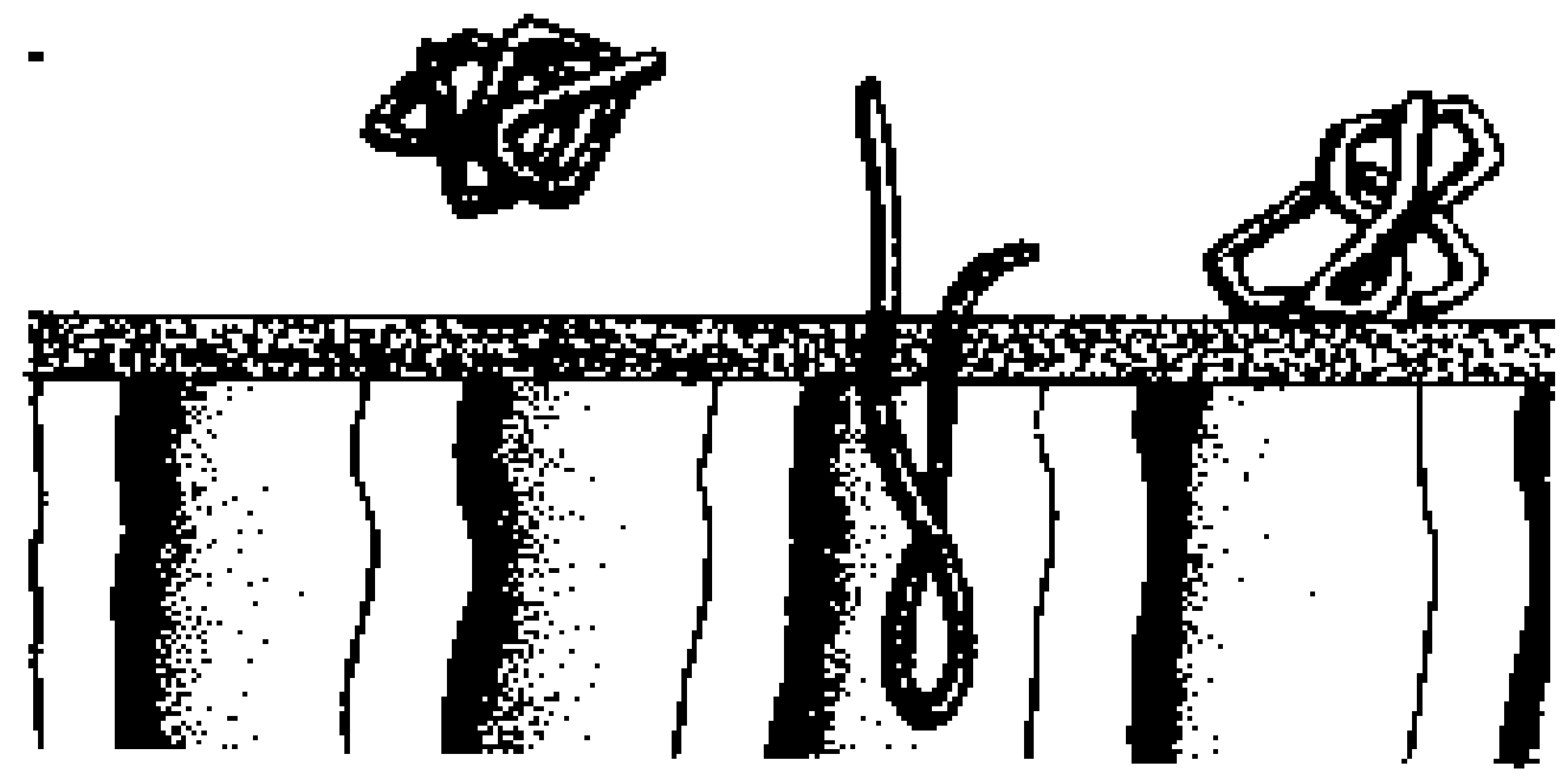

Figure (2.13)—Schematic of Solute Molecules At the Membrane Surface (After Cheryan (1986).) 
constituents in the feed solution. However, two membranes made of the same material will not necessarily have the same solute rejections. Membrane rejections can vary between different membrane configurations. For example, a tubular membrane will not necessarily have the same rejection as a hollow fiber, regardless of the material of construction. [Cheryan, 1986]

\section{Concentration Polarization}

The formation of a concentration boundary layer of rejected solute molecules on the membrane surface has a major affect on permeate water quality. Changes in operational parameters will impact the thickness and density of the boundary layer. For example, increasing the feed solute concentration will result in an increase in the thickness and density of the boundary layer. Therefore, a greater number of rejected solute molecules accumulate at the membrane surface and a greater probability of solute permeation through the membrane exists. Similarly, an increase in the transmembrane pressure will increase the density of the concentration boundary layer. A greater amount of rejected solute molecules at the membrane surface again increases the probability of solute permeation through the membrane. However, an increase in membrane rotational speed will cause an increase in hydraulic turbulence at the membrane surface. Hydraulic turbulence creates a shearing action, which scours accumulated solute molecules from the membrane surface. Therefore, the density and thickness of the concentration boundary layer, as well as the amount of solute molecules at the membrane surface, are decreased. A subsequent decrease in solute permeation through the membrane is observed. 


\section{Limitations Of Conventional Cross-Flow Ultrafiltration Systems}

A major limitation in the application of conventional UF membrane systems (e.g., tubular, hollow fiber, etc.) for the treatment of industrial wastewaters has been the low permeate flux observed at high solute concentrations. With time, a solute boundary layer forms as solute molecules accumulate on the membrane surface due to convective mass transport. This accumulation of solute molecules at the membrane surface is referred to as concentration polarization and is shown schematically in Figure (2.11). If the accumulated solute layer is sufficiently thick, a decrease in permeate flow rate will occur. The buildup of solute molecules at the membrane surface is reduced by back diffusion of solute molecules from the region of high concentration near the membrane surface to the region of lower solute concentration in the bulk solution. The solute boundary layer thickness can be further reduced by providing enhanced hydraulic turbulence to "scour" the accumulated solute from the membrane surface. In conventional membrane systems the hydraulically induced turbulence is provided by recycling a large portion ( 98\%) of the concentrate back to the membrane unit producing high liquid velocities $(\sim 5 \mathrm{~m} / \mathrm{s})$ near the membrane surface. These large velocities increase turbulence which reduces the thickness of the solute boundary layer. However, conventional systems are unable to maintain the high velocities necessary to minimize the deleterious effects of the solute boundary layer on permeate flux because of the difficulty in pumping viscous liquids at high flow rates as the concentrate is thickened with treatment time. [Murkes and Carlsson 1988, Reed et al. 1997] 


\section{MODELING PERMEATE FLUX}

In this section, the Hagen-Poiseuille model for pressure-controlled permeate flux; the osmotic pressure model; the resistance in series model; and the thin-film model are presented and discussed. Additionally, hydraulic turbulence modeling in both conventional and mechanically enhanced membrane separation systems is presented and discussed.

\section{Pressure-Controlled Region--Hagen-Poiseuille Model}

Many attempts at modeling permeate flux have been presented in the literature, though none of which have been entirely satisfactory at predicting flux response to changes in operational parameters (feed concentration, pressure, temperature, etc.) The theoretical relationship between permeate flux, transmembrane pressure, and feed concentration is presented in Figure (2.11). At low feed concentrations and low transmembrane pressures, the Hagen-Poiseuille model for stream-line flow through channels in an ideal membrane system is generally accepted as the best description of fluid flow through a microporous membrane:

$$
\mathrm{J}=\varepsilon \mathrm{r}^{2}\left(\Delta \mathrm{P}_{\mathrm{T}}-\Delta \pi\right) / 8 \mu \Delta \mathrm{x}
$$

where $\mathrm{J}=$ permeate flux, $\varepsilon=$ membrane surface porosity, $\mathrm{r}=$ mean pore radius, $\Delta \mathrm{P}_{\mathrm{T}}=$ hydraulic pressure difference between the feed and the permeate, $\Delta \pi=$ osmotic pressure difference between the feed and the permeate, $\mu=$ viscosity of fluid permeating the membrane, and $\Delta \mathrm{x}=$ membrane thickness. The osmotic pressure term is generally small compared to the applied pressure in ultrafiltration due to the relatively high molecular 
weights of the retained solutes [Cheryan 1986]. In this case, $\Delta \pi \ll \Delta \mathrm{P}_{\mathrm{T}}$ and Equation (2.8) can be simplified:

$$
\mathrm{J}=\varepsilon \mathrm{r}^{2} \Delta \mathrm{P}_{\mathrm{T}} / 8 \mu \Delta \mathrm{x}
$$

Several assumptions are made when using the Hagen-Poiseuille model [Bird et al. 1960]: (1) The flow through the pore channel is laminar. This assumption was validated statistically and experimentally by Cheryan (1986). (2) The permeate is incompressible (3) Permeate flow is independent of time ("steady state" conditions.) (4) The permeate fluid is Newtonian (i.e., the shear force per unit area is linearly proportional to the negative of the velocity gradient). (5) End-effects are negligible (i.e., the flow is "fully developed"). Additionally, the fluid flowing through the pores is generally considered to be contaminant-free water. Therefore, it is customary to use the viscosity of water as the $\mu$ term in Equation (2.9) when modeling permeate flux as a function of applied pressure [Cheryan 1986]. The viscosity of water at several common operating temperatures is presented in Table (2.2) [Weast 1976].

In the Hagen-Poiseuille model, the permeate flux is controlled directly by the transmembrane pressure and inversely by the permeate fluid viscosity. Since the viscosity of a fluid is directly proportional to the solids concentration in the fluid and inversely proportional to the fluid temperature, an increase in temperature will result in an increased permeate flux while an increase in feed concentration will result in a decrease in permeate 
Table (2.2)--Viscosity Of Water At Several Common Operating Temperatures [Weast 1976].

\begin{tabular}{|c|c|}
\hline Temperature $\left({ }^{\mathbf{0}} \mathbf{C} / \mathbf{}^{\mathbf{F}}\right)$ & Viscosity of water $(\mathbf{c P})$ \\
\hline $20 / 68$ & 1.002 \\
\hline $25 / 77$ & 0.8904 \\
\hline $30 / 86$ & 0.7975 \\
\hline $35 / 95$ & 0.7194 \\
\hline $40 / 104$ & 0.6529 \\
\hline $45 / 113$ & 0.5960 \\
\hline $50 / 122$ & 0.5468 \\
\hline $55 / 131$ & 0.5040 \\
\hline $60 / 140$ & 0.4665 \\
\hline
\end{tabular}


flux. As the transmembrane pressure or feed concentration are increased, the permeate flux will become independent of pressure due to the effects of a buildup of solute molecules at the membrane surface. This situation will be discussed in the pressure independent section.

\section{The Osmotic Pressure Model}

An osmotic pressure results from the high solute concentration at the membrane surface. In general, the concentration of solute at the membrane surface if a function of convective transport of solute molecules from the bulk feed solution to the membrane surface and the back diffusion of solute molecules from the membrane surface to the feed solution, as presented schematically in Figure (2.12). When the osmotic pressure due to solute accumulation at the membrane surface is not negligible when compared to the applied pressure, Equation (2.8) is used to model the permeate flux. In this case, it is assumed that the deviation from clean water flux is due only to the osmotic pressure at the membrane surface and not to other phenomena such as increased hydrodynamic resistance due to accumulated solute molecules. Additionally, the relationship between osmotic pressure and solute concentration is presented in Equation (2.10).

$$
\Delta \pi=\Xi C^{n}
$$

where $\Xi$ and $n=$ empirically determined constants and $C=$ solute concentration at the membrane surface.

The osmotic pressure model has been tested in only a few cases. The primary limitation in modeling permeate flux using the osmotic pressure model a lack of osmotic 
pressure data in the form of Equation (2.10). However, in ultrafiltration applications, osmotic pressure is typically small compared to applied pressures and is typically neglected in the modeling of macromolecular solutes (e.g., oil-in-water emulsions and dairy proteins). [Cheryan 1986]

\section{The Resistance Model}

The resistance model uses Darcy's Law presented in Equation (2.11) as a basis for describing permeate flux decline in terms of a system's overall resistance to hydraulic flow. Using this approach, the concentrated solution of macromolecules at the membrane surface is viewed as a physical barrier to hydraulic flow. The resistance of this boundary layer can be divided into and treated as the sum of several individual resistances, analogous to the "resistance in series" concept in heat transfer. The permeate flux in the resistance model is described by:

$$
\mathrm{J}=\Delta \mathrm{P} / \mu\left(\mathrm{R}_{\mathrm{m}}+\mathrm{R}_{\mathrm{bl}}+\mathrm{R}_{\mathrm{a}}\right)
$$

where $\mathrm{R}_{\mathrm{m}}=$ the intrinsic resistance of the membrane, $\mathrm{R}_{\mathrm{bl}}=$ the resistance of the concentration boundary layer, and $\mathrm{R}_{\mathrm{a}}=$ the resistance of the adsorbed solute layer. In the case of a contaminant free clean water feed with a virgin membrane, the resistance of the membrane can be determined using Equation (2.4) by measuring permeate flux as a function of transmembrane pressure under the assumption that $R_{b l}$ and $R_{a}$ are zero. [Jonsson and Tragardh 1990] 


\section{Mass Transfer-Controlled Region--The Thin-Film Model}

As the transmembrane pressure or the solute concentration are increased, or the hydraulic turbulence in the system is decreased, the flux becomes independent of pressure due to a buildup of high solute concentration at the membrane surface, relative to the bulk solute concentration. In the thin-film model the permeate flux in the pressure independent ("mass transfer-controlled") region uses the concept of concentration polarization as a basis to describe the asymptotic flux-pressure relationship shown schematically in Figure (2.11). The permeate flux in the thin film model is described by:

$$
\mathrm{J}=(\mathrm{D} / \delta) \ln \left(\mathrm{C}_{\mathrm{m}} / \mathrm{C}_{\mathrm{b}}\right)
$$

A lumped parameter approach, based on mass transfer considerations, is used to estimate the limiting permeate flux. The solute boundary layer is idealized as a thin liquid film in which mass transport takes place under steady state conditions, where resistance to mass transfer is governed by molecular diffusion normal to the membrane surface. [Lipp et al. 1984]

\section{Mechanically Enhanced UF Systems}

Membrane fouling and the subsequent decline in permeate flux is a major barrier preventing the wider application of membrane technology. Although significant progress has been made to improve the chemical, mechanical, and thermal properties of membrane materials, there has not been a corresponding development of methods to combat the accumulation of solute molecules at the membrane surface and subsequent fouling of the membranes. As a consequence, the performance of new membranes rapidly degrades to that of conventional membrane filter systems. To address the problem of flux decline due 
to accumulation of solute molecules at the membrane surface, several mechanically enhanced UF modules have been designed. Primarily, two general types of mechanically enhanced UF systems have been reported on in the literature: (1) the rotating concentric cylinder and (2) the stacked rotary disk systems which improve upon the conventional tubular and plate and frame configurations, respectively. In each of these systems, a highshear is induced at or near the membrane surface by rotating the membrane or a surface parallel to the membrane.

In conventional membrane systems, maximum surface velocities of approximately $15 \mathrm{ft} / \mathrm{s}(4.6 \mathrm{~m} / \mathrm{s})$ are possible while surface velocities of $60 \mathrm{ft} / \mathrm{s}(18 \mathrm{~m} / \mathrm{s})$ are typical in rotary systems. It is possible to treat extremely concentrated wastes with high-shear rotary UF systems because the cleaning action is effectively decoupled from the pressurization/feed recirculation by rotating the membrane surface at high speeds. In high-shear rotary UF systems, the pump is needed only to provide transmembrane pressure (the driving force) and a small amount of recirculation while the membrane rotation induces turbulence needed to minimize the thickness of the concentration boundary layer [Murkes and Carlsson 1988, Reed et al. 1997].

A schematic of a typical concentric cylinder module is presented in Figure (2.14) [Belfort et al. "Part 1," 1993]. Under specific conditions which will be detailed in a following section, toroidally shaped Taylor vortices will form in the annulus between the inner and the outer cylinders creating turbulence at the membrane surface. The formation of these Taylor vortices, in addition to the primary high-shear flow through the membrane module help to 


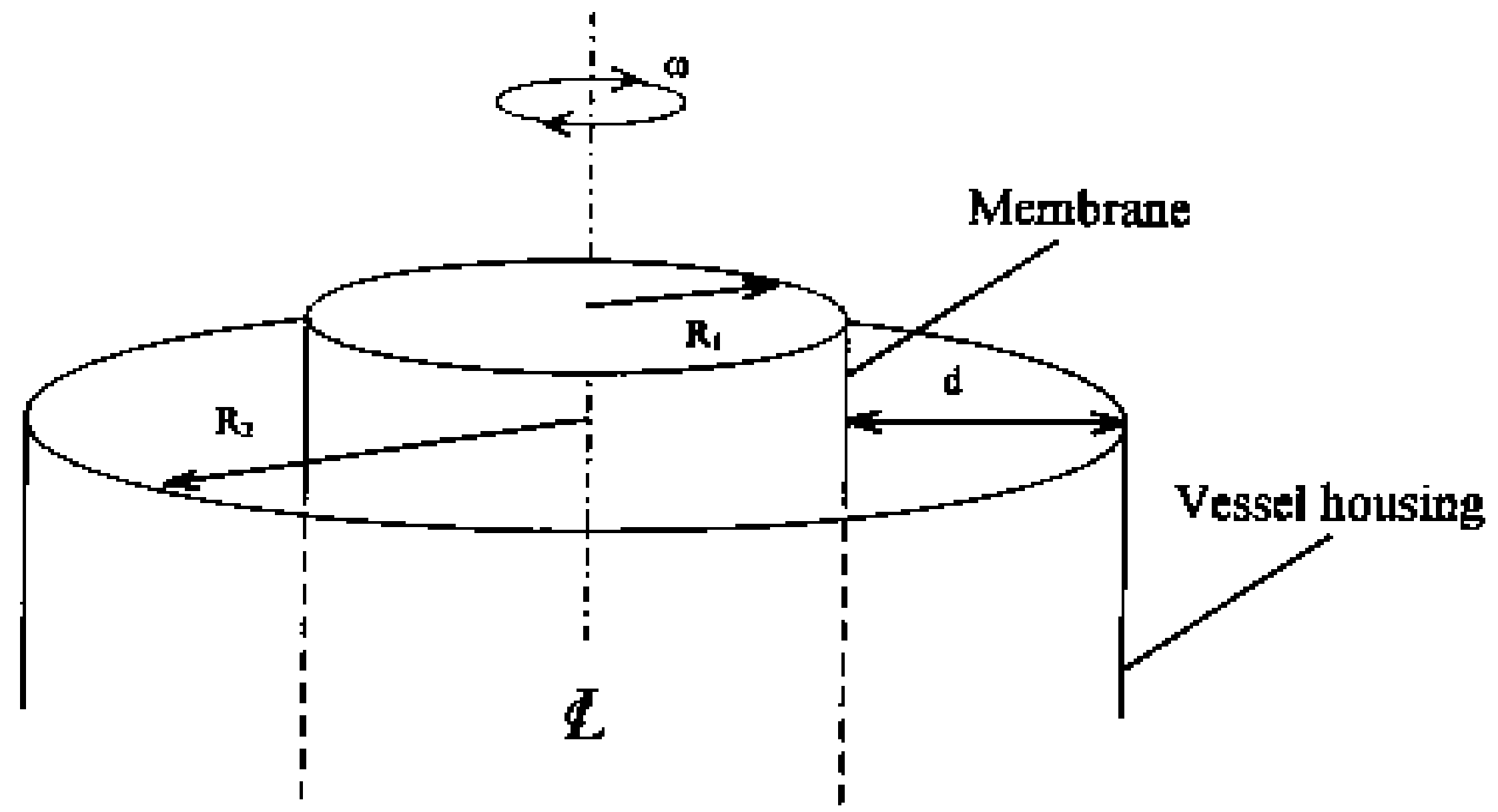

Figure (2.14)—Schematic Of A Typical Concentric Cylinder Module. 
maintain a satisfactory permeate flux by minimizing the accumulation of solute molecules at the membrane surface. Rotating concentric cylinder systems have been limited to research applications for two reasons: (1) the shear force is the same at every point along the surface of the rotating cylindrical membrane, and (2) the annular space between the membrane and vessel wall has been found to be a suitable environment for rejected solute species to react with the liquid being filtered (e.g., a catalyst, an enzyme, adsorbents, etc.) In the second case, the filtration unit can also function as a chemical reactor [Belfort 1988].

In a typical rotary stacked disk UF system, membranes are attached to a series of multiple support plates separated by solid disks mounted on a central shaft. In a rotary stacked disk UF system, the membrane plate remains stationary while the solid disk rotates to induce turbulence which minimizes the accumulation of solute molecules at the membrane surface. In some cases, small vanes have been added to the rotating disks to provide additional turbulence. Multiple disks can be connected in series or in parallel. Typically, a series arrangement is used in thickening/dewatering operations and a parallel arrangement is used when clarification is the principle objective of the process [Murkes and Carlsson 1988].

In rotary disk systems, the membrane-containing plates have traditionally remained stationary while the solid spacer disks are rotated due to difficulties in module design and operation such as devising an adequate sealing system for permeate collection through a rotating body. One recent variation on the rotary disk design is the SpinTek high-shear rotary ultrafiltration unit which has been studied by Reed et al. (ASCE 1997). 
In the SpinTek system, a series of round membrane disks packs are set on a hollow rotating shaft inside a cylindrical housing. A schematic of a high-shear rotary UF pressure vessel is presented in Figure (2.15). The fluid stream enters the membrane chamber under pressure and is distributed across the membrane surface by centrifugal and hydraulic action. Permeate is forced through the membrane, is collected through the hollow rotating shaft, and is discharged. The concentrate exits at the edge of the membrane pack and is returned to the feed tank to be further concentrated.

In a high-shear rotary UF unit, the rotation of the membrane disk is used to induce the hydraulic turbulence required to minimize the thickness of the concentration boundary layer. To enhance the effect of hydraulic turbulence, static turbulence promoters ("wagon wheels") are located on both sides of the membrane disk pack to prevent vortex formation.

A less common mechanically enhanced UF system uses recirculating conicallyshaped "conditioning shuttles", illustrated in Figure (2.16), to remove deposits from the membrane surface. Multiple shuttles are connected to one another via a flexible cable. Shuttles are then forced through a tubular filtration device using the hydraulic flow of the feed solution. The conditioning shuttle system has not been commercialized and no data on its performance have been presented in the literature [Benson 1994].

Flow Conditions In Rotary Membrane Systems

In a rotating concentric cylinder UF membrane system, a high-shear is achieved by using the rotation of the inner cylinder (containing a porous membrane) to form Taylor 


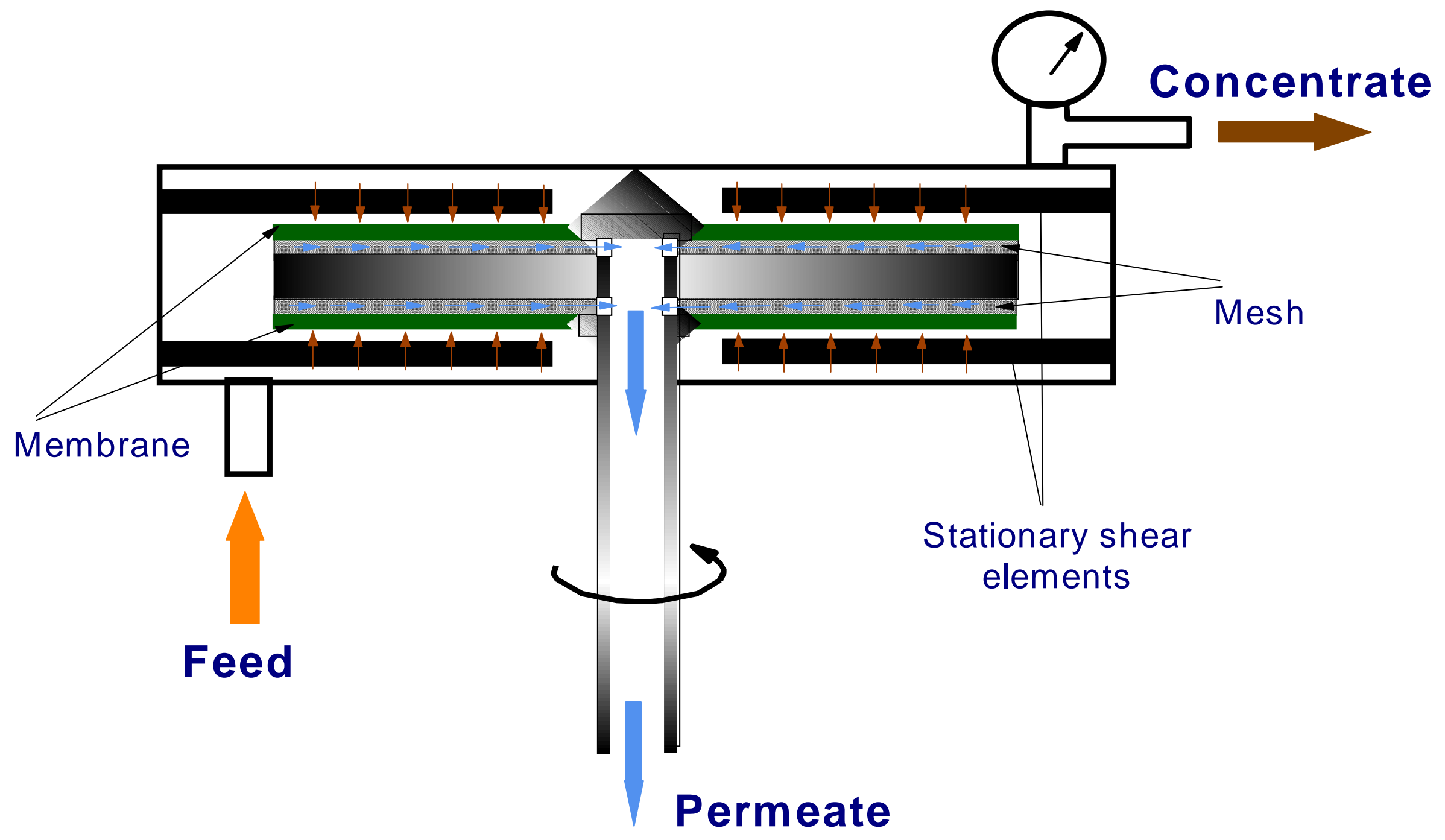

Figure (2.15)—Schematic Of A High-Shear Rotary UF Pressure Vessel. 


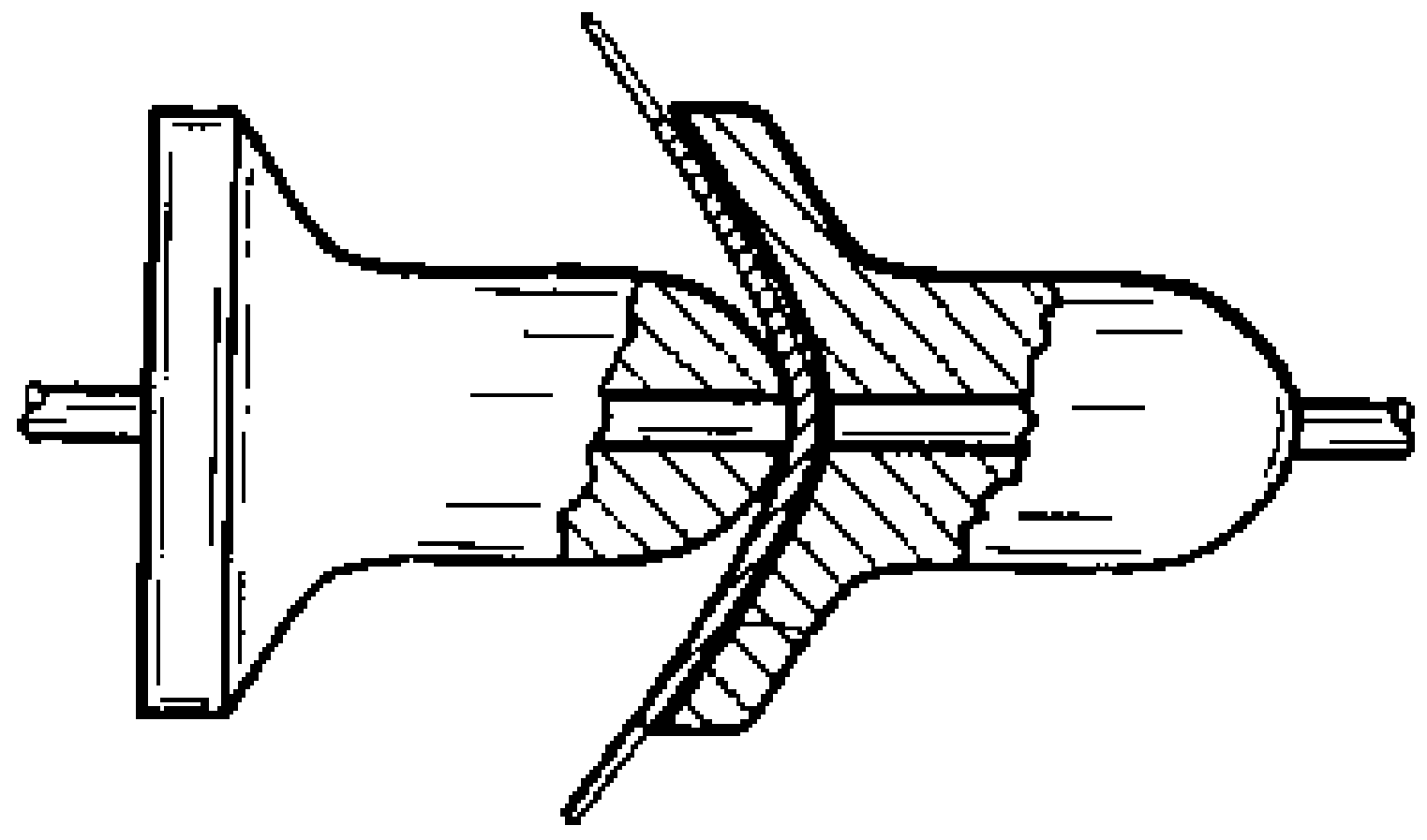

Figure (2.16)—Illustration Of "Conditioning Shuttles." 
vortices in the annular gap between the membrane surface and the vessel wall. For the geometry illustrated in Figure (2.13), the Taylor Number, Ta, is defined as:

$$
\mathrm{Ta}=\left(\omega \mathrm{R}_{1} \mathrm{~d} / \mu\right)\left[2 \mathrm{~d} /\left(\mathrm{R}_{1}+\mathrm{R}_{2}\right)\right]^{1 / 2}
$$

where $\omega=$ membrane rotational speed, $R_{1}=$ inner cylinder radius, $R_{2}=$ outer cylinder radius, $\mathrm{d}=$ annular gap width, and $\mu=$ feed fluid viscosity. When the Taylor Number exceeds the critical value given in Equation (2.13) by increasing the membrane rotational speed, cellular vortex patterns known as a "Taylor Vortices" will be formed. The critical Taylor Number, $\mathrm{Ta}_{\text {crit }}$, in the system was defined by Lieberherr (1978) as:

$$
\mathrm{Ta}_{\text {crit }}=41.1+13.1 \mathrm{~d} / \mathrm{R}_{1}
$$

A the membrane rotational speed is increased further, the flow becomes turbulent. Typically the transition to turbulent flow in rotating concentric cylinder modules occurs at Ta $\geq 400$ [Murkes and Carlsson 1988].

In a rotary disk UF membrane system, a high-shear is achieved by rotating the membrane surface. The flow in the rotary disk system rotary disk system is characterized by two Reynolds Numbers: (1) in the axial direction between the membrane disk and the vessel housing presented in Equation (2.15) and (2) in the radial direction presented in Equation (2.16).

$$
\begin{aligned}
& \operatorname{Re}_{\mathrm{s}}=\omega \mathrm{s}^{2} / \nu \\
& \operatorname{Re}_{\mathrm{r}}=\omega \mathrm{r}^{2} / \nu
\end{aligned}
$$

where $\omega=$ rotational speed, $\mathrm{s}=$ spacing between the membrane and the housing, $\mathrm{r}=$ membrane radius, and $v=$ kinematic viscosity of feed solution. For a given feed solution, 
the maximum Reynolds Number occurs at the outer radius of the rotating disk and increases with rotational speed [Murkes and Carlsson 1988].

Ketola and McGrew (1968) identified four distinct flow regimes for a partially wetted rotating disk based on the radial Reynolds Number and the spacing ratio between the disk and the stationary wall. The membrane radius and spacing in a high-shear rotary UF system are presented in Figure (2.17). Ketola and McGrew assumed there is no radial net flow in the development of this set of flow conditions.

Case I--Laminar flow and narrow gap.

$$
\operatorname{Re}_{\mathrm{s}}<4 \text { and } \operatorname{Re}_{\mathrm{r}}<2 \times 10^{5}
$$

The laminar boundary layers are merged to produce a shear rate which varies inversely with spacing, $s$. The shear stress, $\tau$, in this case is:

$$
\tau_{\mathrm{I}}=\mu \omega \mathrm{r} / \mathrm{s}
$$

where $\mu=$ viscosity of the feed solution and $\rho=$ density of the feed solution.

Case II--Laminar flow and wide gap.

$$
\operatorname{Re}_{\mathrm{s}}>4 \text { and } \operatorname{Re}_{\mathrm{r}}<2 \times 10^{5}
$$

Under Case II hydraulic flow condition separate boundary layers are formed and the shear rate is independent of spacing, s. The shear stress in this case is:

$$
\tau_{\mathrm{II}}=1.81 \rho v^{1 / 2}(\mathrm{~K} \omega)^{3 / 2} \mathrm{r}, 0<\mathrm{K}<1
$$




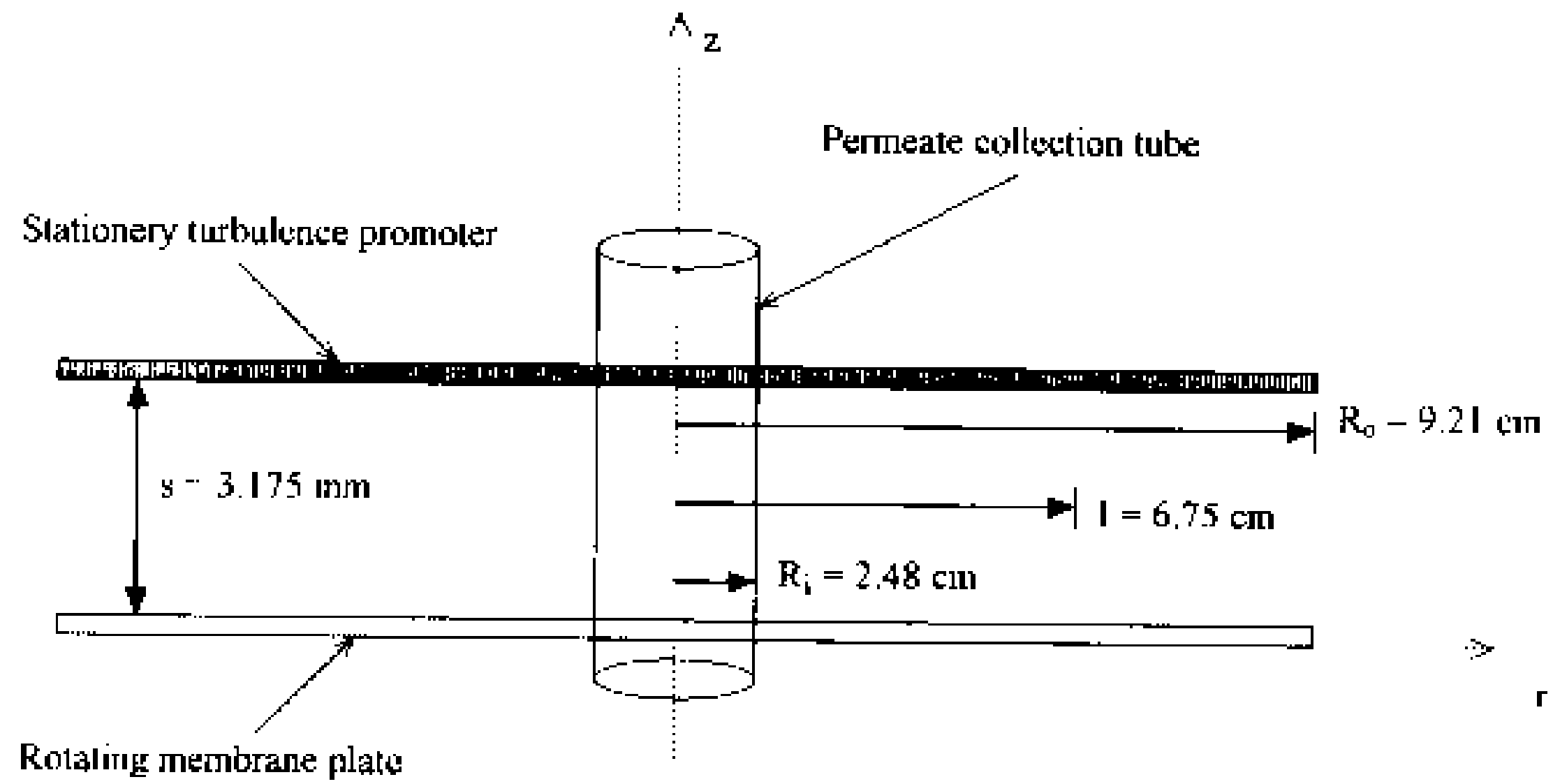

Figure (2.17)—The Membrane Radius And Spacing In A High-Shear Rotary UF System. 
The area between the boundary layers moves like a solid body with a rotational speed of $\mathrm{K} \omega$, where $0<\mathrm{K}<1$ ( $\mathrm{K}$ is a dimensionless constant).

Case III--Turbulent flow and narrow gap.

$$
\operatorname{Re}_{\mathrm{r}}>2 \times 10^{5} \text { and } \mathrm{s} / \mathrm{r}<0.05
$$

The hydraulic flow under this condition is characterized by two merged turbulent boundary layers. The shear stress in this case is:

$$
\tau_{\mathrm{III}}=0.008 \rho(\omega \mathrm{r})^{7 / 4}(\mathrm{v} / \mathrm{s})^{1 / 4}
$$

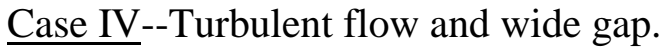

$$
\operatorname{Re}_{\mathrm{r}}>2 \times 10^{5} \text { and } \mathrm{s} / \mathrm{r}>0.05
$$

Under Case IV hydraulic flow conditions, two boundary layers are formed separated by a turbulent core region. The shear stress in this case is:

$$
\tau_{\mathrm{IV}}=0.057 \rho v^{1 / 5}(\mathrm{~K} \omega)^{9 / 5} \mathrm{r}^{8 / 5}, 0<\mathrm{K}<1
$$

Murkes and Carlsson reported that $\mathrm{K}$ values ranging from 0.4 to 0.5 gave good agreement with experiments for the laminar flow wide gap (Case II) and turbulent flow wide gap (Case IV) flow conditions. Additionally, when eight radial vanes were installed on a rotating membrane disk, a $\mathrm{K}$ value of $0.90(\underset{\sim}{(1.0)}$ was reported under turbulent flow wide gap (Case IV) conditions which gave a large increase in shear stress and a corresponding increase in filter flux.

In rotary membrane separation systems, the hydraulic turbulence represented by the Reynolds Number can be further expressed in terms of the membrane rotational speed, $\omega$, or the shear stress, $\tau$. Thus, the power law relationship can be expressed as: 


$$
\mathrm{J}=f(\operatorname{Re} \text { or } \omega \text { or } \tau)^{\mathrm{n}}
$$

The value of $\mathrm{n}$ in Equation (2.40) can be determined by calculating the slope of a line through the data in a log-log plot of permeate flux in the mass transfer-controlled region versus Reynolds Number, the shear stress, or membrane rotational speed. Typical values of $\mathrm{n}$ were presented previously in Table (2.4) for both laminar and turbulent hydraulic flow conditions.

Permeate Backpressure In A High-Shear Rotary UF System

In the high-shear rotary UF system illustrated previously in Figures (2.14) and (2.16), the central aim is to use membrane rotation to induce turbulence at the membrane surface to reduce the thickness of the flux-defeating solute boundary layer. However, in addition to providing turbulence in the system, membrane rotation has an impact on the net transmembrane pressure. The effects of membrane rotation on net transmembrane pressure in rotary concentric cylinder UF systems were reported on by Belfort et al. ("Part 1," 1993) and Dolcek et al. (1995). In the high-shear rotary UF system, a non-uniformly distributed backpressure results from centrifugal force exerted on permeate due to membrane rotation. The permeate backpressure for a given membrane rotational speed and radius is expressed as:

$$
\mathrm{p}_{\text {back }}=\left[\rho(\omega \mathrm{r})^{2}\right] / 2
$$

where $\omega=$ rotational speed, $r=$ outer membrane radius, and $\rho=$ feed solution density. The maximum and minimum permeate backpressures are found at the maximum and minimum disk radii, respectively. The average backpressure due to centrifugal force on 
the permeate in the high-shear rotary UF system can be determined using the radius of gyration for a flat rotating ring as the radial term in Equation (2.22).

$$
\text { pback-avg }=\left[\rho(\mathrm{I} \omega)^{2}\right] / 2
$$

where $\mathrm{I}=$ radius of gyration (an average radial property of a rotating body) for a flat rotating ring. The explicit form of the radius of gyration term for the rotating ring is [Weast 1976]:

$$
\mathrm{I}=\left[\left(\mathrm{R}_{\mathrm{i}}^{2}+\mathrm{R}_{\mathrm{o}}^{2}\right) / 2\right]^{1 / 2}
$$

where $R_{i}=$ inner radius and $R_{o}=$ outer radius. The maximum permeate backpressure, p pack-max , is observed along the outer radius of the rotating disk, $\mathrm{R}_{\mathrm{o}}$ :

$$
p_{\text {back-max }}=\left[\rho\left(\omega R_{\mathrm{o}}\right)^{2}\right] / 2
$$

In the high-shear rotary UF system, the average transmembrane pressure, $\mathrm{p}_{\text {tmp-avg, }}$ is the difference between the gauge pressure, $p_{\text {gauge, }}$ and the average permeate back pressure, pback-avg:

$$
p_{\text {tmp-avg }}=p_{\text {gauge }}-p_{\text {back-avg }}
$$

An important aspect of the pressure/rotation relationship in a high-shear rotary disk system is the need to set the system operating parameters to ensure a positive net transmembrane pressure:

$$
p_{\text {gauge }}>p_{\text {back-max }}
$$

where $p_{\text {back-max }}=$ maximum permeate backpressure (observed at the maximum membrane radius) for a given membrane rotational speed. If the condition in (2.27) is not met, the 
driving force will reverse, forming a vacuum which can force fluid from the permeate side of the membrane back to the concentrate side. Vacuum formation could result in serious operational difficulties such as membrane delamination and a loss in operating efficiency.

\section{Mechanically Enhanced Ultrafiltration Case Studies}

In this section, general studies on the operation and modeling of mechanically enhanced UF systems are discussed. Studies which deal specifically with the mechanically enhanced ultrafiltration of oily wastewaters are discussed separately.

To minimize the effects of concentration polarization, Lopez-Leiva (1980) investigated the application of a rotating concentric cylinder membrane, a rotary disk and frame (RDF) module, and a "Roto-Shear" (RS) unit. The concentric cylinder membrane system was similar to the unit used by Belfort, et al. ("Part 1" and "Part 2" 1993) and Dolecek et al. (1995). The RDF module used whole plates to separate a series of stacked disk membranes and the RS unit used rotating blades situated in between membrane disks to enhance turbulence at the membrane surface. In a study using a feed solution of bovine serum albumin (BSA) at 0.5 to $1.0 \%$, Lopez-Leiva reported that the concentric cylinder module was effective at reducing the effects of concentration polarization to near negligible values at low feed solute concentrations and high cross-flow velocities. Experiments were conducted at membrane rotational speeds ranging from $\sim 250$ to 4250 $\mathrm{rpm}$, at $15^{\circ} \mathrm{C}$, and pressures ranging from 0.3 to $0.5 \mathrm{MPa}$ ( $\sim 4$ to $116 \mathrm{psig}$ ). 
Lopez-Leiva (1980) examined the performance of the two rotary disk units using a feed solution of skim and whole milk. Lopez-Leiva reported that high concentrations ( $45 \%$ total solids) could be obtained using both the RDF and the RS rotary disk units. However, when the energy consumption per liter of milk processed as a function of total solids content was calculated, Lopez-Leiva reported that the RDF module was more energy efficient compared with the RS unit. Lopez-Leiva suggested that for the RS unit, which used blades placed between the membrane disks as turbulence promoters, vortices and wakes may have resulted in large energy losses. These energy losses were not transmitted to the concentration boundary layer and as a result, the energy did not go to improve mass transfer but was dissipated as heat.

Belfort et al. ("Part 1," 1993) performed a study to examine the performance of a rotating annular filter similar in design to the module presented schematically in Figure (2.14). In these experiments, the transmembrane pressure was corrected to account for the centrifugal pressure exerted on the permeate using a narrow-gap solution to the Navier-Stokes equations for azimuthal flow in an annulus and Darcy's Law for flow through a porous wall on the inner rotating cylinder. Rotational speeds ranged from 500 to $4,000 \mathrm{rpm}$ and transmembrane pressures ranged from $\sim 20$ to $100 \mathrm{kPa}$ ( $\sim 3$ to $14.5 \mathrm{psig}$ ). These experiments were conducted using a $0.45 \mu \mathrm{m}$ polysulfone microfilter and a 100 $\mathrm{kDa}$ ultrafilter with feed solutions of cell cultures (bovine serum albumin, ovalbumin, and lactoglobulins).

The governing equation derived by Belfort et al. ("Part 1," 1993) for the wall velocity (or flux) is presented in Equation (2.28). The authors considered the components 
of the flow to be idealized (i.e., approximately laminar), without vortices, and weakly coupled to solve for the permeate flux in terms of the annular pressure and the permeate pressure:

$$
\mathrm{v}_{\mathrm{w}}=(\kappa / \Delta \mathrm{x})\left[\mathrm{P}_{1}-\rho \omega^{2} \mathrm{R}_{1}{ }^{2}\left(\mathrm{~d} / 3 \mathrm{R}_{1}\right)\right]
$$

where $\kappa=$ membrane permeability, $\Delta x=$ membrane thickness, $P_{1}=$ pressure at the surface of the tubular membrane, $\rho=$ fluid density, $\omega=$ angular velocity of the rotating cylinder, $\mathrm{R}_{1}=$ radius of the tubular membrane, and $\mathrm{d}=$ distance between the inner tube filter and the vessel wall. Equation (2.28) is actually the lowest order expansion of a much more complicated expression. In the simplification it was assumed that the membrane thickness and annular spacing were much less than the membrane radius, $\mathrm{R}_{1}$ ("thin slit" approximation.") The Navier-Stokes equations were first simplified assuming the velocity in the radial direction was small. Additionally, the small gap assumption mentioned earlier was used. The alternative approach resulted in an equation with the same functional dependence as in Equation (2.28). In Equation (2.29), Belfort et al. ("Part 1," 1993) identified the centrifugal pressure due to membrane rotation, $\Delta \mathrm{P}_{\text {cent }}$.

$$
\Delta \mathrm{P}_{\text {cent }} \simeq\left(\rho \omega^{2} \mathrm{R}_{1}^{2}\right)\left(\mathrm{d} / 3 \mathrm{R}_{1}\right)
$$

Belfort et al. ("Part 1," 1993) then conducted experiments using commercially available cell culture media to empirically verify the flux-pressure model presented in Equation (2.28). In these experiments, large differences between theoretically predicted and experimental data were observed when the transmembrane pressure was not corrected to account for centrifugal pressure effects. However, when the centrifugal pressure 
correction was employed, the data from these experiments collapsed onto a single straight line $\left(r^{2}=0.9485\right)$ as predicted by the governing equation given in Equation (2.28). The data in these experiments were entirely in the pressure-controlled region.

Since Belfort et al. did not have a detailed understanding of the influence of the solute deposition on the membrane surface as a function of transmembrane pressure, a phenomenological approach was used to study the effects of rotation on membrane fouling. Belfort et al. assumed the resistance to flow was directly dependent on transmembrane pressure and inversely proportional to the rotational speed. It was concluded that the transmembrane pressure (which itself is a function of rotation as presented in Equation (2.47)) can be used to: (1) clean the membrane by creating a highshear by forcing fluid through the vessel channel; (2) overcome the added resistance to flow created by pore narrowing, constriction, and plugging; and (3) to overcome a serial resistance to flow which results from a build-up of a gel/cake layer through upstream solute deposition on the membrane surface.

In a related paper, Belfort et al. ("Part 2," 1993) used the resistance in series approach to determine the serial resistance introduced by the accumulation of solute at the membrane surface and plotted the resistance against the transmembrane pressure. Experiments were conducted using a $0.45 \mu \mathrm{m}$ polysulfone microfilter and a $100 \mathrm{kDa}$ ultrafilter with feed solutions of deioniozed water, and $1 \mathrm{wt} . \%$ solid suspensions of styrene-divinyl-benzene and styrene-polytoluene. An annular gap to membrane radius ratio of 0.1102 and transmembrane pressures ranging from $\sim 0$ to $30 \mathrm{kPa}$ ( 0 to $\sim 4.4 \mathrm{psi}$ ) 
were used in the study. A steady decrease in the resistance of the colloidal layer with increasing membrane rotation was observed. It was hypothesized that the increase in rotation had the effect of decreasing the thickness of the concentration boundary layer which resulted in a decreased hydrodynamic resistance.

In a theoretical assessment of rotating filter performance similar to those of Belfort et al. ("Part 1" and "Part 2," 1993), Dolecek et al. (1995) solved the NavierStokes and continuity equations with Darcy's Law to analyze the flow of a pure fluid through an annulus with a rotating inner porous wall. Dolecek et al. then compared the results with data presented by Belfort et al. ("Part 2," 1993). However, Dolecek et al. neglected the formation of Taylor vortices by restricting the experiments to flows below the critical Taylor number (i.e., the number above which Taylor vortices will form) to eliminate additional complications associated with accounting for the annular flow and subsequent pressure effects caused by the formation of Taylor vortices. For the rotating concentric cylindrical filtration system, Belfort et al. ("Part 2," 1993) presented the following expression for the Taylor Number, Ta:

$$
\mathrm{Ta}=\left[\mathrm{R}_{1} \omega\left(\mathrm{R}_{2}-\mathrm{R}_{1}\right) / \mathrm{v}\right]\left[\left(\mathrm{R}_{2}-\mathrm{R}_{1}\right) / \mathrm{R}_{1}\right]^{1 / 2}
$$

where $R_{1}=$ membrane radius, $R_{2}=$ vessel radius, and $v=$ kinematic viscosity of the feed solution. Since the shear rate at the surface of the membrane depended upon the rate of membrane rotation, the Taylor number was also a function of rotation.

Dolecek et al. identified three pressure terms as contributing to the overall driving force, $\Delta \mathrm{P}_{\text {total }}$, in the system given in Equation (2.31): (1) a centrifugal term $\left(\Delta \mathrm{P}_{\text {cent }}\right),(2) \mathrm{a}$ 
radial convection term $\left(\Delta \mathrm{P}_{\text {radial }}\right)$, and (3) a Darcy's Law term $\left(\Delta \mathrm{P}_{\text {Darcy }}\right)$ which accounted for the hydrodynamic resistance of the membrane. It was reported that in general, the pressure drop due to a change in the radial velocity was negligible when compared with the centrifugal and Darcy's Law terms (i.e., a weak axial pressure drop).

$$
\Delta \mathrm{P}_{\text {total }}=\Delta \mathrm{P}_{\text {cent }}+\Delta \mathrm{P}_{\text {radial }}+\Delta \mathrm{P}_{\text {Darcy }}
$$

Of particular interest in the rotating system was the "centrifugal pressure" term which was different than the expression developed in the simplified version of the rigorous development present by Belfort et al. ("Part 1," 1993).

$$
\Delta \mathrm{P}_{\text {cent }}=\left\{\left(\rho \omega^{2}\right) *\left[\mathrm{R}_{1}{ }^{2}-\left(\mathrm{R}_{1}-\Delta \mathrm{x}\right)^{2}\right]\right\} / 2
$$

where $\rho=$ fluid density, $\omega=$ angular velocity of the rotating cylinder, $R_{1}=$ radius of the tubular membrane, and $\Delta \mathrm{x}=$ membrane thickness.

$$
\Delta \mathrm{P}_{\text {radial }}=\left\{\left(\rho \mathrm{q}^{2} / 8 \pi^{2}\right)\left[1 /\left(\left(\mathrm{R}_{1}-\Delta \mathrm{x}\right)^{2}\right)-\left\{1 / \mathrm{R}_{1}^{2}\right)\right]\right\}
$$

where $\mathrm{q}=$ permeate flow rate.

$$
\Delta \mathrm{P}_{\text {Darcy }}=(\mathrm{q} / 2 \pi \kappa) \ln \left[\mathrm{R}_{1} /\left(\mathrm{R}_{1}-\Delta \mathrm{x}\right)\right]
$$

where $\kappa=$ membrane permeability. (Permeate side pressure was assumed to be zero.) The form of the Darcy's Law term is the same as shown by Belfort et al. ("Part 1" 1993).

The results of the theoretical model developed by Dolecek et al. were compared with experimental flux-pressure data observed by Belfort et al. ("Part 1," 1993). The experimental flux-pressure data were taken over a 20 to $\sim 90 \mathrm{kPa}(2.9$ to $\sim 13 \mathrm{psi}$ ) range in which the data were entirely in the pressure-controlled region. The authors attributed the discrepancy to a hydrostatic backpressure in the permeate chamber ( 2 to $3 \mathrm{kPa})$ which 
tended to reduce the experimentally observed net $\Delta \mathrm{P}_{\text {total }}$. The formation of complex flow patterns such as Taylor vortices may have been an additional contribution to the difference between the model developed by Dolecek et al. and the experimental results observed by Belfort et al. ("Part 2," 1993).

\section{MEMBRANE TREATMENT OF OILY WASTEWATERS}

In this section, general properties of oil-in-water emulsions and oil waste waters is presented and discussed. Additionally, an overview of the membrane treatment of oily wastewaters is presented and discussed.

\section{Characteristics of Oil-In-Water (O/W) Emulsions And Oily Wastewaters}

The metal-working industry produces large volumes of oily wastewaters. Examples of metal-working processes which utilize large quantities of emulsified oils for lubrication, cooling, and quenching include metal rolling, forming, grinding, and cutting operations. Concentrations of oil and grease $(\mathrm{O} / \mathrm{G})$ vary widely across the metal-working industry. A summary of typical $\mathrm{O} / \mathrm{G}$ concentration ranges for selected industrial wastewaters is presented in Table (2.3) [Bennett 1988].

Oil is typically found in three forms in industrial processes: (1) free oil consisting of discrete oil droplets, usually $5 \mu \mathrm{m}$ in diameter or larger; (2) agglomerated oil made up of discrete oil droplets $5 \mu \mathrm{m}$ or less in diameter; and (3) emulsified oil in which fine globules of oil are dispersed in water by the addition of a chemical "emulsifier" [Laemmle 1992]. Unstable emulsions containing agglomerated oil can be chemically broken. However, stable emulsions require 
Table (2.3)--Typical O/G Concentration Ranges For Selected Industrial Wastewaters [Bennett 1988].

\begin{tabular}{|c|c||}
\hline \hline Industry & O/G concentration range (mg/L) \\
\hline \hline Food processing & $100-1,000$ \\
\hline Textile (wool processing ) & $10-50$ \\
\hline Petroleum refining & $100-1,000$ \\
\hline Metal rinse water & $10-1,000$ \\
\hline Metal fabrication & $10,000-150,000$ \\
\hline Metal rolling & $4,000-50,000$ \\
\hline Commercial laundry & $100-2,000$ \\
\hline
\end{tabular}


increasingly sophisticated treatment techniques to meet more stringent effluent discharge guidelines. Traditionally, these wastewaters are treated using chemical addition followed by gravity separation techniques. Often, the effluent from chemical addition solid liquid separation processes required additional treatment prior to discharge. In contrast, pressure-driven membrane separation systems can typically treat the waste in a single step and are gaining acceptance as an alternative treatment technology.

Emulsions are mixtures of either simple or compounded oils with water, stabilized by the use of emulsifiers and other additives such as secondary stabilizers/surfactants. Emulsified oils are widely used when both cooling and lubricating properties are required, whereas straight oils are used when lubrication is the main concern and cooling capacity is a minor factor. A schematic of an emulsified oil droplet is presented in Figure (2.18) (after Burke (1991)). It is possible for an emulsion to have a net positive or neutral charge, depending upon the properties of the emulsifier. However, anionic emulsifiers are typically used in the cooling/lubricating fluids common in the metal-working industry. In a typical $\mathrm{O} / \mathrm{W}$ emulsion, oil globules are sequestered by a polar emulsifier which consists of an oil soluble end and a negatively charged oleophobic/hydrophilic end. Typical oil globule sizes range from $\sim 0.2$ to $10 \mu \mathrm{m}$ [Laemmle 1992]. However, the size of oil globules in an $\mathrm{O} / \mathrm{W}$ emulsion is not discrete throughout a solution but varies over some distribution which depends primarily on the ratio of oil to emulsifier, rather than on the oil content [Lipp, et al. 1988]. Since the typical size of the emulsified oil droplet is similar in size to, or greater than, the wavelength of visible light, 


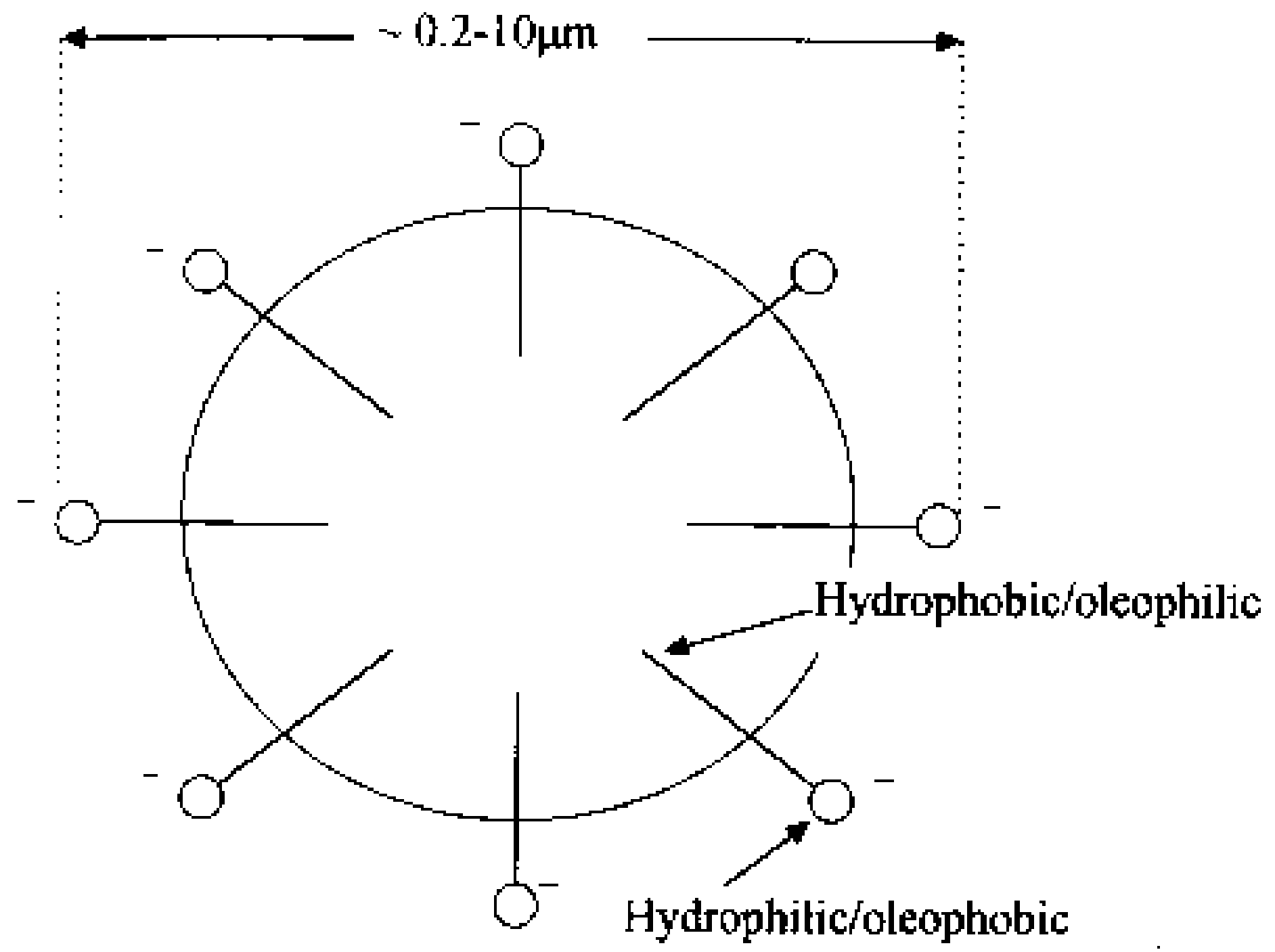

Figure (2.18)—Schematic Of Emulsified Oil Droplet (After Burke (1991).) 
these emulsions appear milky white.

The stability and lubricating characteristics of an $\mathrm{O} / \mathrm{W}$ emulsion used in metalworking processes are carefully balanced to optimize the operational properties of the solution. $\mathrm{O} / \mathrm{W}$ mixtures are thermodynamically unstable (i.e., state of lowest free energy is total phase separation). As a result, oil tends to separate from water in solution. To keep the oil droplets sequestered, emulsifiers and secondary stabilizers (chemicals which concentrate at the oil-water interface and prevent oil globule coalescence) are used. Since the hydrophilic end of the O/W emulsion is negatively charged, the net surface charge of the emulsified oil droplet is negative. The emulsified oil droplets tend to stay dispersed due to electrostatic repulsion between the emulsified oil droplets. The emulsion is then said to be kinetically stable because the state of lowest free energy of the emulsion is still total separation. In general, stable emulsions tend to have a smaller average globule size distribution than unstable emulsions. Emulsion stability can vary over a wide range and is affected by the chemical nature of the oil and the concentrations of emulsifier and additives in the solution.

The ability of an $\mathrm{O} / \mathrm{W}$ emulsion to provide sufficient lubrication is tied to the availability of the oil phase to lubricate (lubrication improves with oil availability). Oil availability is controlled by both the emulsion stability and oil content. Typically, oil availability increases as oil content increases and as emulsion stability decreases. Less stable emulsions are subject to rapid, often unpredictable, declines in stability over short periods of time. The transient behavior can lead to severe operating problems in metalworking processes such as rolling mill operations. Additionally, as the oil content in an 
emulsion increases, cooling capacity decreases because there is less water available for cooling. As a result, the stability and oil concentration of an emulsified metal-working fluid must be balanced to meet both the lubricating and cooling needs of each specific metal-working process, leading to the heterogeneous nature of wastewaters from the metal-working industry.

Since O/W emulsions are kinetically stable but not thermodynamically stable, the "batch life" of the coolant/lubricant package must also be taken into consideration. New emulsions are typically the most stable and have the least oil available for lubrication. Over time, the maximum performance of the emulsion is reached as emulsifiers are (1) depleted through biological degradation and (2) as debris generated in the emulsion provides nucleation sites for oil globule coalescence. However, as the emulsion is degraded further, the emulsion becomes so unstable as to be rendered useless and a new batch of emulsion must be introduced into the process. Emulsions that are initially more stable generally have a longer batch life than emulsions which are initially less stable [Laemmle 1992].

Typical $\mathrm{O} / \mathrm{W}$ emulsions used in rolling mill operations consist of complex proprietary mixtures of fatty acids and mineral oils, emulsifiers, biocides, stabilizers, antifoaming agents, and rust inhibitors. The main O/G components of these rolling mill oils are fatty acids and mineral oils. Wastewaters in rolling mill processes contain $\mathrm{O} / \mathrm{G}$ from coolant sprayed on metal strips to dissipate heat and lubricate the material sheets. Other contributions to $\mathrm{O} / \mathrm{G}$ concentrations in these wastewaters come from non-emulsified "tramp oils" which leak into the waste stream from ancillary mechanical operations (e.g., 
hydraulic fluids). Typical coolant/lubricant solutions used in metal-working operations contain approximately 5 to $10 \%$ emulsified oil and approximately 90 to $95 \%$ water [Laemmle 1992]. A common base oil package consists of $\sim 80 \%$ mineral oil and $\sim 20 \%$ additives (e.g., emulsifiers, anti-foaming agents, biocides, etc.). [Lee et al. 1984].

\section{Overview of Membrane Treatment of Oily Wastewaters}

Ultrafiltration has been successfully applied to the treatment of concentrated oily wastes from metal-working processes such as sheet rolling and die cutting. UF has been used to treat wastewaters high in oil, grease, and solids while reducing the volume of waste sent for disposal, while at the same time recovering alkaline process cleaner for reuse. The advantages of UF treatment of oily wastewaters over more traditional methods such as chemical addition solid/liquid separation include: (1) chemical additives are not necessary; (2) higher oil removal efficiencies; and (3) relative ease of use over other techniques [Lee et al. 1984].

\section{Conventional Ultrafiltration Systems}

Pinto (1978) reported that UF treatment of a waste cutting oil emulsions reduced the volume of wastewater by 95 to $98 \%$ and concentrated solids and oil as much as $60 \%$. Cheryan (1986) summarized the application of UF technology to the treatment of a mixed oily wastewater containing mineral oils, paraffin wax, oleic acid, and other oily constituents. The mixed waste stream was stabilized to minimize membrane fouling by adding a nonionic surfactant. The waste was then treated using Abocor tubular

membranes $\left(72 \mathrm{~m}^{2}, 776 \mathrm{ft}^{2}\right.$ membrane area). The membrane pore size/molecular weight 
cut off (MWCO) was not specified. At an average pressure of $\sim 2.5$ atm (37 psi), an average permeate flux of $41 \mathrm{~L} / \mathrm{m}^{2}-\mathrm{h}\left(24 \mathrm{gal} / \mathrm{ft}^{2}-\mathrm{d}\right)$ was observed while maintaining a permeate water quality acceptable for direct discharge to the sanitary sewer. Oil and grease concentrations were reduced from $3,530 \mathrm{mg} / \mathrm{L}$ in the feed to $35 \mathrm{mg} / \mathrm{L}$ in the effluent. Further, a 94\% reduction in COD was observed after UF treatment of the oily wastewater.

Due to the heterogeneous nature of oily wastewaters, the type of membrane materials and MWCOs of membranes used to treat oily wastes varies widely. It was reported that, in general, oily wastewaters are not very demanding on membrane materials since the $\mathrm{pH}$ of the feed solution is typically less than 10 . However, since the exact composition of oily wastewaters can vary widely, a membrane material should ideally be able to withstand the wide range of $\mathrm{pHs}$ which may be encountered in the aggressive caustic and acidic cleaning procedures necessary to restore permeate flux. Common membrane materials used in the treatment of oily wastewaters include (1) polymeric (e.g., polyvinyliden fluoride (PVDF), polyamide, and polysulfone), (2) cellulose, and (3) ceramic.

Lipp et al. (1988) reported on the UF treatment of oil-in-water emulsions in which oil rejections of $99.9 \%$ were observed. Regenerated cellulose (5 and 30k MWCO), polysulfone (30k MWCO), polyacrylic (10k MWCO), and polyamide (100k MWCO) membranes were used in the experiments. Oil rejection was independent of membrane material and operating pressure. TOC rejections ranging from 96 to $98 \%$ were observed. 
Elevated TOC concentrations in the permeate, relative to the concentration of oil in the permeate, were attributed to the presence of lower molecular weight soluble additives (e.g., surfactants and stabilizers) that passed through the membrane. Although oil rejections of $99.9 \%$ were observed for each membrane material, Lipp et al. (1988) reported that regenerated cellulose membranes were the most easily cleaned. In contrast, Lipp et al. (1988) reported that the polysulfone membranes were not able to be cleaned effectively. Additionally, Canepa et al. (1988) used a tubular polysulfone membrane with a 20k MWCO to concentrate an oily waste down to $30 \%$ of its original volume. However, Canepa et al. did not discuss the durability/cleanability of the polysulfone membrane after treating the oily wastewater.

Mahdi and Skold (1991) used a tubular fluropolymer membrane with a MWCO of 20k to reduce the effluent mineral oil content of a synthetic water-based metal grinding coolant from $44 \mathrm{mg} / \mathrm{L}$ prior to processing to $1 \mathrm{mg} / \mathrm{L}$ after UF treatment. Bodzek and Konieczy (1992) used polyacrylonitrile (PAN) and polyvinyl chloride (PVC) tubular membranes with MWCOs ranging from 20 to 50k to treat a waste oil emulsion. Bodzek and Konieczy reported that COD retentions of greater than $95 \%$ were observed using these membranes. Additionally, polymeric membranes with MWCOs ranging from 5 to 50k were used by Zaidi et al. (1992) to remove oil successfully from oilfield brine. Reed et al. (Sep. Sci. \& Tech. 1997) reported the effective use of a 100k MWCO PVDF tubular membrane to concentrate a waste metal-working fluid to a maximum of 116 times its initial oil concentration of $\sim 0.2$ to $0.5 \%$. 
Oil volume reductions of 95 to $99 \%$ were reported by Bodzek and Konieczny (1992) for the treatment of a metal-working lubricant using polyacrylonitrile (PAN) and polyvinyl chloride (PVC) tubular membranes with MWCOs ranging from 20 to 50k in a pilot-scale UF setup. Reed et al. (Sep. Sci \& Tech. 1997) reported on the pilot-scale treatment of an aluminum manufacturer's $\sim 0.2$ to $0.5 \%$ oil and grease wastewater using tubular PVDF UF membranes. Volume reductions ranging from 94 to $99 \%$ were reported in these experiments.

Membrane surface properties can also affect permeate flux and quality. Electrostatic repulsion between the membrane and the contaminant can be used to enhance waste solute retention and increase flux. For example, a membrane with a negative surface charge can be used to treat a waste containing a negatively charged contaminant. Reed et al. (Sep. Sci. \& Tech. 1997) reported that a negatively charged tubular membrane with a MWCO of 120k had a higher flux and similar permeate quality when compared with a membrane having a neutral surface charge and an MWCO of 100k when treating a $\sim 0.2$ to $0.5 \% \mathrm{O} / \mathrm{G}$ wastewater. In this case, Reed et al. hypothesized that the electrostatic repulsion between the negative charge of the membrane surface and the negative charge of the emulsified oil droplets decreased the solute boundary layer thickness and oil adsorption on the membrane surface which improved the membrane performance.

\section{Mechanically Enhanced Ultrafiltration Systems}

In an applied study on the ultrafiltration of oily emulsions, Vigo et al. (1985) investigated the effects of pressure, rotational speed, and oil concentration on permeate 
flux using freshly prepared cutting oil emulsions in a rotating concentric cylinder membrane system similar to the unit presented schematically in Figure (2.13). The main characteristics of the experimental unit used by Vigo et al. were: $40 \mathrm{~cm}$ height, $10 \mathrm{~cm}$ diameter, membrane area of $700 \mathrm{~cm}^{2}$, and variable membrane/wall gap ranging from 2.5 to $3.3 \mathrm{~mm}$. A maximum tangential rotation speed of $12 \mathrm{~m} / \mathrm{s}$ was possible with the rotating concentric cylinder system. Vigo et al. identified the formation of a solute boundary layer and subsequent fouling phenomena as the primary limiting factor in the efficient operation of ultrafiltration systems, particularly when treating highly viscous concentrated oily wastewaters. Vigo et. al suggested that the effects of the added hydrodynamic resistance of concentration boundary layer can be minimized by carefully controlling the hydrodynamic conditions in the UF unit through proper selection of membrane rotation, applied pressure, gap between membrane and vessel, and the "roughness" of the vessel wall.

In the rotating concentric cylinder membrane unit studied by Vigo et al., the formation of Taylor vortices was identified as the primary mechanism of high-shear formation at the membrane surface. Additionally, Vigo et al. reported that permeate flux was affected by the "roughness" of the vessel wall. When a 270 and a 140 mesh were used to line the vessel wall an increase in permeate flux over the non-lined case was observed. The 270 mesh, which produced a rougher surface compared to the 170 mesh, was more effective in enhancing the permeate flux. However, the exact mechanism of 
flux enhancement was not identified and the authors cited the need for a more systematic study of the effects of vessel roughness on permeate flux.

Vigo et al. noted a more rapid transition from the pressure-controlled to the mass transfer-controlled regions as membrane rotational speed was decreased from a tangential velocity of 9 to $5 \mathrm{~m} / \mathrm{s}$. The rapid transition to pressure independent flux was attributed to the increased accumulation of solute molecules at the membrane surface as the shear was decreased.

Additionally, the gap width between the membrane and the vessel wall was found to have an influence on the permeate flux, particularly at lower membrane rotational speeds. The authors attributed the effects of gap width to the different hydrodynamic conditions and the different size of the Taylor vortices. Vigo et al. also compared the results obtained with the rotating concentric cylinder UF module to a traditional tubular UF system and reported that the rotary unit gave greater operational flexibility over the tubular UF system and attributed the advantage to the decoupling of shear and feed pressure. Vigo et al. recommended operating at low feed pressures and high rotational speeds to minimize the thickness of the concentration boundary layer. In each case, this group observed oil rejections greater than $99.9 \%$ throughout the experiments with the rotating annular system.

Reed et al. (ASCE 1997) reported on an applied study of the treatment of an aluminum manufacturers oily wastewater using a high-shear rotary UF system. A comparison of a $100 \mathrm{k}(\sim 0.01 \mu \mathrm{m}$ pore size $)$ polyvinlyden fluoride membrane with a 
ceramic $\left(\mathrm{TiO}_{2} / \mathrm{Al}_{3} \mathrm{O}_{2}, 0.11 \mu \mathrm{m}\right.$ average pore size $)$ membrane in a high-shear rotary UF system was conducted and it was concluded that the performance of the ceramic membrane was superior to that of the PVDF with regard to flux quantity and quality as well as membrane cleaning/durability. In the experiments, an oily wastewater was concentrated from 5 to as high as $70 \%$ using a $0.11 \mu \mathrm{m}$ ceramic membrane in the highshear rotary UF system.

Reed et al. (ASCE 1997) also reported on the effects of feed temperature on permeate flux. When the feed temperature was increased from 100 to $140{ }^{0} \mathrm{~F}$, for a $20 \%$ waste metal-working fluid feed treated in a high-shear rotary operated at 1,750 rpm, permeate flux increased by $\sim 45 \%$. However, the theoretically anticipated flux increase, based on the change in permeate viscosity, was $\sim 21 \%$. Reed et al. attributed the larger than anticipated increase in permeate flux to an increase in solute molecule diffusivity at elevated temperature. Thus, using the ceramic membrane, the researchers were able to make better use of a strong relationship between feed temperature and permeate flux by operating the system at higher temperatures than would have been possible with a PVDF membrane. Additionally, the ceramic membrane was able to be cleaned effectively (using $\mathrm{NaOH} / \mathrm{surfactant}$ solution to adjusted to $\mathrm{pH} 2.2$ ) and reused after treating concentrated oily wastewaters (up to $70 \%$ oil in the feed), whereas it was not possible to fully recover a satisfactory permeate flux when treating the same type of wastewater using the PVDF membrane. In this case, a new PVDF membrane was required for each experiment. 
Reed et al. (ASCE 1997) also reported on the effects of membrane rotational speed and hydraulic turbulence on permeate flux in a high-shear rotary UF system. The $\mathrm{n}$ value was consistent with the range of 0.8 to 1.2 for turbulent hydraulic flow condition reported by Cheryan (1986). Reed et al. noted that when the membrane rotational speed was decreased from 1,750 to $1,000 \mathrm{rpm}$, permeate flux decreased. It was hypothesized that the decrease in permeate flux was due to an increase in the thickness of the solute concentration boundary layer at the membrane surface. When the membrane rotational speed was increased from 1,000 to 1,500 an increase in permeate flux was observed. The authors hypothesized that the increase in permit flux was due to a decrease in the thickness of the solute concentration boundary layer. A decrease in permeate flux was observed when the rotational speed was decreased from 1,500 to $1,250 \mathrm{rpm}$ (similarly to the first decrease in rotational speed from 1,750 to $1,000 \mathrm{rpm}$ ). When the membrane rotational speed was increased from 1,250 to the pre-excursion value of $1,750 \mathrm{rpm}$, the permeate flux increased. However, the permit flux did not recover to the initial preexcursion level. Reed et al. hypothesized that the gel layer was sufficiently stable to reduce the effects of increased hydraulic turbulence as the membrane rotational speed was raised. Thus, Reed et al. recommended running the high-shear rotary UF system at the highest rotational speed possible. 


\section{CHAPTER 3.0 INVESTIGATIVE APPROACH}

In this chapter, the experimental design and materials and methods used in this study are described. Quality assurance/quality control measures are also discussed. In this chapter, applied pressures are designated as $\mathrm{P}_{\mathrm{i}}$, membrane rotational speeds are designated as $\mathrm{R}_{\mathrm{j}}$, metal-working (MW) fluid concentrations are designated as $\mathrm{O}_{\mathrm{k}}$, and oil concentrations are designated as $\mathrm{OC}_{\mathrm{k}}$.

\section{EXPERIMENTAL DESIGN}

\section{Experimental Conditions}

The effects of membrane rotational speed and applied pressure on permeate water quality were studied in individual experiments. MW fluid concentrations ranged from 5 to $40 \%$, applied pressures ranged from 15 to $75 \mathrm{psig}$, and membrane rotational speeds of 1150 and $1750 \mathrm{rpm}$ were studied. Metal-working fluid/membrane rotational speed combinations and applied pressure ranges investigated in this study are presented in Table (3.1). The lowest concentration, 5\% MW fluid, was selected to approximate typical concentrations in the direct treatment of waste MW fluids in an industrial setting [Lammale 1992, Reed et al. 1997]. The maximum membrane rotational speed of the high-shear rotary UF unit was $1,750 \mathrm{rpm}$. The lowest rotational speed of $1150 \mathrm{rpm}$ was chosen to obtain data in the mass transfer-controlled region for the low MW fluid concentration experiments. The average and maximum permeate backpressures over the range 
Table (3.1)--Metal-Working Fluid/Membrane Rotational Speed Combinations and Pressure Ranges Investigated in this Study.

\begin{tabular}{|c|c|c|c|c|c|c|}
\hline $\begin{array}{c}\text { Metal-working fluid } \\
\text { concentration }\left(\mathrm{O}_{\mathrm{k}}\right), \% \rightarrow \\
\text { [Applied pressure range }\left(\mathrm{P}_{\mathrm{i}}\right), \mathrm{psig} \text { ] } \\
\text { Rotational speed }\left(\mathrm{R}_{\mathrm{j}}\right), \mathrm{rpm} \\
\downarrow\end{array}$ & 5 & 10 & 15 & 20 & 30 & 40 \\
\hline 1750 & $\begin{array}{c}5 \% \\
{[25-75 \mathrm{psig}]} \\
1750 \mathrm{rpm} \\
\end{array}$ & $\begin{array}{c}10 \% \\
{[25-75 \mathrm{psig}]} \\
1750 \mathrm{rpm} \\
\end{array}$ & $\begin{array}{c}15 \% \\
{[20-75 \mathrm{psig}]} \\
1750 \mathrm{rpm} \\
\end{array}$ & $\begin{array}{c}20 \% \\
{[20-70 \mathrm{psig}]} \\
1750 \mathrm{rpm} \\
\end{array}$ & $\begin{array}{c}30 \% \\
{[25-70 \mathrm{psig}]} \\
1750 \mathrm{rpm} \\
\end{array}$ & $\begin{array}{c}40 \% \\
{[25-70 \text { psig] }} \\
1750 \mathrm{rpm} \\
\end{array}$ \\
\hline 1150 & $\begin{array}{c}5 \% \\
{[25-75 \mathrm{psig}]} \\
1150 \mathrm{rpm}\end{array}$ & $\begin{array}{c}10 \% \\
{[25-75 \mathrm{psig}]} \\
1150 \mathrm{rpm}\end{array}$ & $\begin{array}{c}15 \% \\
{[15-70 \mathrm{psig}]} \\
1150 \mathrm{rpm}\end{array}$ & $\begin{array}{c}20 \% \\
{[15-70 \mathrm{psig}]} \\
1150 \mathrm{rpm}\end{array}$ & $\begin{array}{c}30 \% \\
{[15-70 \mathrm{psig}]} \\
1150 \mathrm{rpm}\end{array}$ & $\begin{array}{c}40 \% \\
{[15-70 \mathrm{psig}]} \\
1150 \mathrm{rpm}\end{array}$ \\
\hline
\end{tabular}


membrane rotational speeds investigated in this study were accounted for to ensure a positive transmembrane pressure across the entire membrane disk. Average and maximum permeate backpressures at typical membrane rotational speeds are presented in Table (3.2). The applied pressure was increased from low to high values to avoid biasing the permeate water quality by initially operating under conditions which are favorable to the accumulation of oil at the membrane surface and subsequent plugging of membrane pores. Data collected during these experiments included: permeate flux; permeate and concentrate $\mathrm{pH}$ and conductivity; and permeate and concentrate temperature. A standardized cleaning procedure was performed and a clean water flux was measured after each experiment.

Steps were taken to minimize variations due to other experimental parameters (e.g., metal-working fluid characteristics, metal-working fluid temperature, and hydraulic flow). Synthetic MW fluids were freshly prepared in each experiment. Experiments were conducted in recycle mode for each $\mathrm{R}_{\mathrm{j}} \mathrm{O}_{\mathrm{k}}$ combination, to minimize the potential effects of changes in feed properties over time (e.g., emulsion breakdown due to high shear and heat) [Cheryan 1986]. Additionally, the potential bias toward decreasing permeate water quality during direct batchdown operation due to membrane fouling at higher MW fluid concentrations was minimized by performing experiments at discrete MW fluid concentrations. To minimize the effects of variable hydraulic turbulence in the each experiment, the system was operated at one membrane rotational speed with a concentrate 
Table (3.2)--Average and Maximum Permeate Backpressures at Typical Rotational Speeds.

\begin{tabular}{||c|c|c||}
\hline $\begin{array}{c}\text { Membrane Rotational Speed, } \\
\mathrm{R}_{\mathrm{j}}(\mathrm{rpm})\end{array}$ & Average $\mathrm{p}_{\text {back }}(\mathrm{psi})$ & Maximum $\mathrm{p}_{\text {back }}(\mathrm{psi})$ \\
\hline 1,750 & 11.1 & 20.6 \\
\hline 1,450 & 7.6 & 14.1 \\
\hline 1,250 & 5.7 & 10.6 \\
\hline 1,150 & 4.8 & 9.0 \\
\hline 850 & 2.6 & 4.9 \\
\hline 500 & 1.0 & 1.8 \\
\hline 250 & 0.3 & 0.5 \\
\hline
\end{tabular}


return flow rate of $1.0 \pm 0.2 \mathrm{gpm}(3.75 \pm 0.75 \mathrm{~L} / \mathrm{min})$. The feed temperature was maintained at $110 \pm 2{ }^{0} \mathrm{~F}$. Additionally, a single ceramic membrane was used $(0.11 \mu \mathrm{m}$ average pore size) for all experiments. A summary of the ceramic membrane characteristics is presented in Table (3.3). This membrane was made of the same material investigated by Reed et al. (ASCE 1997).

\section{MATERIALS AND METHODS}

\section{Experimental Apparatus}

A single disk high-shear rotary ultrafiltration unit was used in this study. A schematic of the membrane vessel and the experimental apparatus are presented in Figures (3.1) and (3.2), respectively. In this system, one 8 inch $(20 \mathrm{~cm})$ round membrane disk pack was set on a hollow rotating shaft inside the vessel housing. The feed stream entered the membrane chamber under pressure and was distributed across the membrane surface by centrifugal and hydraulic action. Applied pressure was supplied by a Grundfos model CRN2 centrifugal pump capable of a maximum feed pressure of $\sim 78 \mathrm{psi}$ (at zero

gpm outlet flow rate). The operating temperature range of the pump was 5 to $250{ }^{0} \mathrm{~F}$ and the original equipment manufacturer single mechanical seal (carbide/tungsten carbide) was retrofitted with a double mechanical seal (tungsten carbide/tungsten carbide). The feed flow and pressure to the vessel were controlled using a bypass valve installed on the pump. The applied pressure was measured using a 100 psi maximum pressure gauge installed on the top of the pressure vessel. The pressure gauge, manufactured by McDaniel Controls, Inc., was graduated in 1 psi increments. The concentrate return flow 
Table (3.3)--A Summary of the Ceramic Membrane Characteristics.

\begin{tabular}{|c|c|}
\hline Manufacturer & TRUMEM \\
\hline Lot number & CRM-011M-TA \\
\hline Chemical composition & $\mathrm{TiO}_{2} / \mathrm{Al}_{2} \mathrm{O}_{3}$ \\
\hline Mean pore size & $0.11 \mu \mathrm{m}$ \\
\hline Maximum pore size & $0.70 \mu \mathrm{m}$ \\
\hline Total membrane area & $0.53 \mathrm{ft}^{2}$ \\
\hline Maximum operating temperature & $>158^{0} \mathrm{~F}$ \\
\hline Maximum operating pressure & $>150 \mathrm{psi}$ \\
\hline Thickness & $240 \mu \mathrm{m}$ \\
\hline Inner radius & $0.975 \mathrm{in}$. \\
\hline Outer radius & $3.625 \mathrm{in}$. \\
\hline Radius of gyration & $2.654 \mathrm{in}$. \\
\hline
\end{tabular}




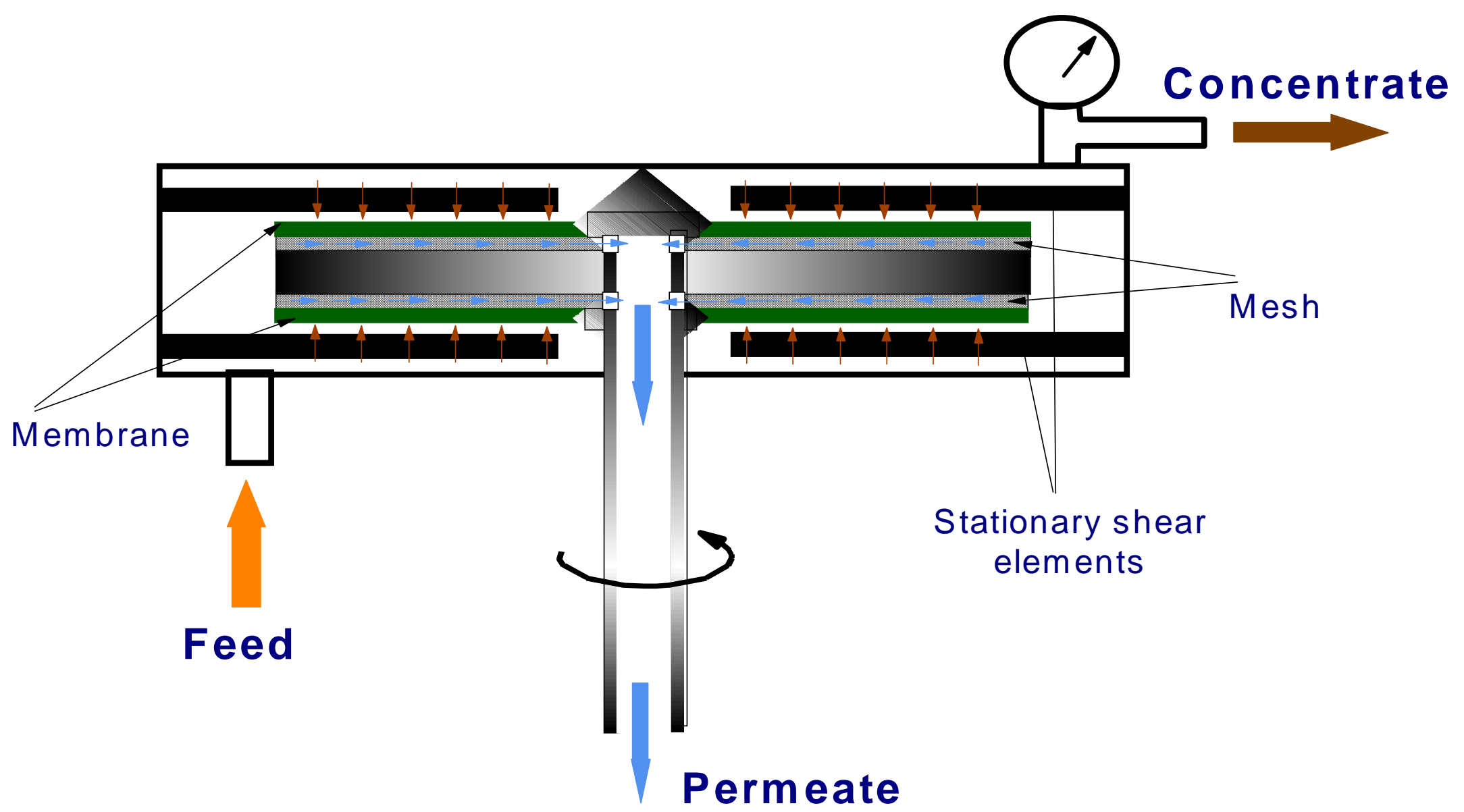

Figure (3.1)—Schematic of the Membrane Vessel. 


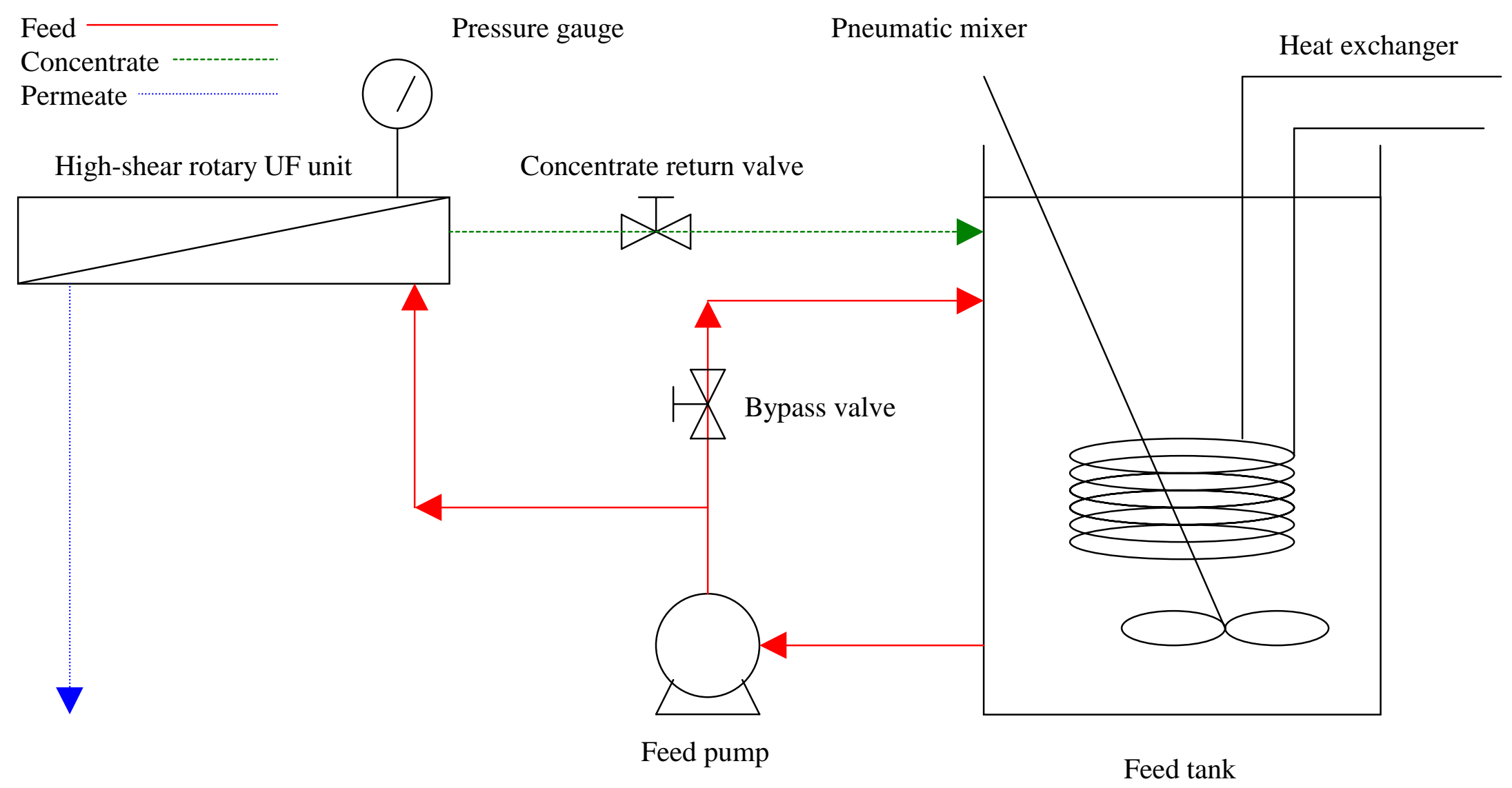

Figure (3.2)—Schematic of the Experimental Apparatus. 
rate was measured using a Signet 8511 turbine flow meter and was maintained at $1.0 \pm$ $0.2 \mathrm{gpm}(3.75 \pm 0.75 \mathrm{~L} / \mathrm{min})$ throughout these experiments by adjusting return flow valve installed on the concentrate return line. Additionally, a shut-off ball valve was added to the concentrate return line so the return flow rate could be measured manually using a graduated cylinder and a stop watch. This procedure was used to periodically check the calibration of the turbine flow meter. The rotation of the membrane disk was used to induce the hydraulic turbulence required to minimize the thickness of the concentration boundary layer. Membrane rotation could be varied from 150 to $1,750 \mathrm{rpm}$. These limiting values were preset by the manufacturer and could not be altered on-site. Feed temperature was maintained at $110 \pm 2{ }^{0} \mathrm{~F}$ by passing tap water through a copper coil heat exchanger located in the feed tank. A Fisher Scientific digital temperature probe was installed in the feed tank to monitor the feed temperature throughout these experiments. An additional Fisher Scientific digital temperature probe was used to measure the temperature of the permeate collected during the permeate flow measurement. A pneumatic mixer was used to maintain feed homogeneity and to aid in temperature control. To minimize the loss of feed volume due to evaporation of water, a plastic lid with an access port for the pneumatic mixer was kept on the feed tank throughout the duration of these experiments. 


\section{Experimental Conduct}

\section{Membrane Cleaning Procedure}

After completing an experiment, the system was stopped and the feed tank and lines were drained. The vessel was opened and physical observations of the membrane's condition were made. Particular attention was paid to the accumulation/formation of an oily ring or a gel-like layer on the membrane surface. Additionally, the membrane was inspected for scratches and tares. The membrane was then reinstalled and a standardized cleaning procedure was used. A summary of the characteristics of the base/surfactant and acid cleaning solutions used in the standardized cleaning procedure is presented in Table (3.4). Dawn dish washing detergent, an EDTA-containing surfactant, was added to the base cleaning solution to complex metals that may have built up on the membrane and to dissolve oils accumulated on the membrane surface. The membrane cleaning procedure is summarized below:

1. The system was flushed with 50 gallons $(190 \mathrm{~L})$ of hot tap water. The membrane was not operated at this time.

2. Five gallons (19 L) of distilled water was adjusted to $\mathrm{pH} 11.8$ using $\mathrm{NaOH}$ from a $1 \mathrm{M}$ stock solution stored with a soda scrubber.

- $200 \mathrm{~mL}$ of $1 \mathrm{M} \mathrm{NaOH}$ was necessary to raise the $\mathrm{pH}$ of the distilled water to 11.8 .

- Additionally, $30 \mathrm{~mL}$ of Dawn detergent was mixed into the water and $\mathrm{NaOH}$ solution.

- The $\mathrm{pH}$ and conductivity of the base/surfactant cleaning solution was measured and recorded.

- The base/surfactant cleaning solution was then heated to $135 \pm 2{ }^{0} \mathrm{~F}$ using the copper coil heat exchanger. 
Table (3.4)--A Summary of the Characteristics of the Base/Surfactant and Acid Cleaning Solutions Used in the Standardized Cleaning Procedure.

\begin{tabular}{|c|c|c||}
\hline & Base/surfactant & Acid \\
\hline $\mathrm{pH}$ & 11.8 & 2.2 \\
\hline conductivity & $3.8 \mathrm{mS} / \mathrm{cm}$ & $8.1 \mathrm{mS} / \mathrm{cm}$ \\
\hline Vol. stock NaOH added $(\mathrm{mL})$ & 200 & $\mathrm{x}$ \\
\hline Vol. Dawn added $(\mathrm{mL})$ & 30 & $\mathrm{x}$ \\
\hline Vol. $\mathrm{H}_{2} \mathrm{SO}_{4}$ added $(\mathrm{mL})$ & $\mathrm{x}$ & 185 \\
\hline
\end{tabular}

$\mathrm{x}=$ Not added to this solution. 
3. The membrane was cleaned by running the base/surfactant cleaning solution through the high-shear rotary UF system for 30 minutes at $1,750 \mathrm{rpm}, 135 \pm 2{ }^{0} \mathrm{~F}$, and $40 \mathrm{psig}$.

- Permeate flux was measured and recorded during the cleaning cycle.

4. The system was drained and flushed with 50 gallons of hot tap water.

- 50 gallons was determined to be the volume necessary to reduce the $\mathrm{pH}$ of the effluent flush water to the $\mathrm{pH}$ of tap water after cleaning with $\mathrm{NaOH}$.

5. Five gallons ( $19 \mathrm{~L})$ of distilled water was $\mathrm{pH}$ adjusted 2.2 using a stock $1 \mathrm{M}$ $\mathrm{H}_{2} \mathrm{SO}_{4}$ solution.

- $185 \mathrm{~mL}$ of $1 \mathrm{M} \mathrm{H}_{2} \mathrm{SO}_{4}$ was necessary to reduce the $\mathrm{pH}$ of the distilled water to 2.2 .

- The $\mathrm{pH}$ and conductivity of the acid cleaning solution was measured and recorded.

- The acid cleaning solution was then heated to $135 \pm 2{ }^{0} \mathrm{~F}$ using the copper coil heat exchanger.

6. The membrane was cleaned by running the acid cleaning solution through the high-shear rotary UF system for 30 minutes at $1,750 \mathrm{rpm}, 135 \pm 2{ }^{0} \mathrm{~F}$, and 40 psig.

- Permeate flux was measured and recorded during the cleaning cycle.

7. The system was drained and flushed with 50 gallons of hot tap water.

- 50 gallons was determined to be the volume necessary to raise the $\mathrm{pH}$ of the effluent flush water to the $\mathrm{pH}$ of tap water after cleaning with $\mathrm{H}_{2} \mathrm{SO}_{4}$.

8. The membrane vessel was opened and physical observations of the membrane's condition were made as described earlier.

9. The membrane was reinstalled and a clean water flux (CWF) was measured at $110 \pm 2{ }^{0} \mathrm{~F} ; 1,750 \mathrm{rpm}$; at three applied pressure from 25 to 75 psig with a 40 $\mathrm{L}$ distilled water feed.

- The initial $\mathrm{pH}$ and conductivity of the distilled water used in the CWF experiment were measured and recorded.

- The average permeate flux versus the average transmembrane pressure was plotted.

- The slope of the line fitted to these data using linear regression analysis was determined and the membrane resistance was calculated as presented in Equation (2.4). 
The standardized cleaning and clean water flux procedure was applied after each experiment and the membrane resistance was tracked throughout the study.

\section{Metal-Working Fluid Experiments}

The high-shear rotary ultrafiltration system was used to treat synthetic emulsified MW fluids at the $\mathrm{R}_{\mathrm{j}} \mathrm{O}_{\mathrm{k}}$ combinations presented previously in Table (3.1). Each discrete $\mathrm{R}_{\mathrm{j}} \mathrm{O}_{\mathrm{k}}$ experiment was conducted in recycle mode, with a total feed volume of $40 \mathrm{~L}$, at a feed temperature of $110 \pm 2{ }^{0} \mathrm{~F}$, and a return flow rate of $1.0 \pm 0.2 \mathrm{gpm}$.

Feed Preparation: A base MW package was used to make the 5 to $40 \%$ metalworking fluid solutions in these experiments. The base MW package consisted of mineral oil, surfactants, film strength additives, and antifoaming agents. However, the specific components of the base MW package cannot be disclosed for proprietary reasons. The density, absolute viscosity, and particle size distributions of each MW fluid concentration were experimentally measured to determine baseline characteristics. The specific procedures for these analyses are described in the Analytical Methods Section.

Approximately $100 \mathrm{~L}$ of distilled water to make the feed oil package solution and cleaning solutions was obtained from West Virginia University Civil and Environmental Engineering Laboratory's house supply. The $\mathrm{pH}$ and conductivity of the distilled water were measured and compared with previously observed values to judge the quality of the water prior to being used in these experiments. The $\mathrm{pH}$ and conductivity ranges of distilled water used to make the feed MW fluid solutions were 6.69 to 7.27 and 1.17 to $6.88 \mu \mathrm{S} / \mathrm{cm}$, respectively. Forty liter feed solutions were then made up in the feed tank by adding the appropriate volume of base MW package to distilled water. The base MW 
package and water volumes added to make each concentration are presented in Table (3.5).

System Operation: Prior to starting the system, the metal-working fluid solution was heated to $110 \pm 2{ }^{0} \mathrm{~F}$ and the pneumatic mixer was turned on to homogenize the feed solution and to aid in heating. The feed temperature was measured using a Fisher Scientific digital temperature probe installed in the feed tank. The pneumatic mixer was run throughout each experiment. A plastic lid with an access port for the pneumatic mixer was kept on the feed tank to minimize the loss of feed volume due to the evaporation of water. The system was then started, the membrane rotational speed was set using the dial and digital control on the membrane unit, and the applied pressure was adjusted to its minimum value by adjusting the bypass valve on the feed pump. Additionally, the concentrate return flow rate, displayed on the Signet flow meter, was stabilized at $1.0 \pm 0.2 \mathrm{gpm}$ using the concentrate return flow valve. When all operational parameters were stabilized, the permeate flux and permeate and concentrate conductivity; $\mathrm{pH}$; and temperature were measured regularly at each applied pressure condition. Flux measurements were made every five minutes for the first fifteen minutes at a pressure condition and then every ten to fifteen minutes thereafter. The permeate flow was measured by disconnecting the permeate line from the feed tank and measuring the volume of permeate collected over a given period of time using a graduated cylinder and a stopwatch. The $\mathrm{pH}$, conductivity, and turbidity of the permeate sample was measured. The permeate temperature was measured using a Fisher Scientific digital temperature probe placed in the graduated cylinder as a permeate sample was being 
Table (3.5)--The Base MW Package and Water Volumes Added to Make Each MW Fluid Concentration.

\begin{tabular}{||c|c|c||}
\hline $\begin{array}{c}\text { Feed MW fluid concentration } \\
(\% \text { base MW package })\end{array}$ & $\begin{array}{c}\text { Volume of base MW } \\
\text { package added (L) }\end{array}$ & $\begin{array}{c}\text { Volume of distilled water } \\
\text { added (L) }\end{array}$ \\
\hline 5 & 2 & 38 \\
\hline 10 & 4 & 36 \\
\hline 15 & 6 & 34 \\
\hline 20 & 8 & 32 \\
\hline 30 & 12 & 28 \\
\hline 40 & 16 & 24 \\
\hline
\end{tabular}


collected. To obtain a representative reading of the permeate temperature, the thermometer probe was agitated in the cylinder prior to measuring the temperature. The concentrate $\mathrm{pH}$ and conductivity were measured every fifteen minutes immediately after measuring permeate parameters. Since each experiment was conducted in recycle mode (i.e., constant $\mathrm{O}_{\mathrm{k}}$ ), all permeate was returned to the feed tank after measuring the flux, temperature, $\mathrm{pH}$ and conductivity.

System Shutdown: When the final applied pressure condition was completed, the membrane rotational speed was turned to the lowest setting of $150 \mathrm{rpm}$, the pump was shut off, and the power to the unit was disconnected. The vessel was opened and physical observations of the membrane's condition were made. Particular attention was paid to the accumulation/formation of an oily ring or a gel-like layer on the membrane surface. Additionally, the membrane was inspected for scratches and tares. The cleaning procedure detailed earlier was applied and a clean water flux was measured after each experiment. Following system cleaning, the entire experimental procedure was repeated again for a constant $\mathrm{R}_{\mathrm{j}} \mathrm{O}_{\mathrm{k}}$ combination as a function of applied pressure.

\section{Analytical Methods}

Prior to beginning an experiment, the $\mathrm{pH}$ and conductivity meters were calibrated using standard solutions. During these experiments, $\mathrm{pH}$ and conductivity were collected and analyzed in triplicate when changes in these parameters were observed.

Permeate Oil and Grease: Permeate oil and grease (O/G) analysis was determined using Standard Methods 503 C, Soxhlet Extraction Method. A 1L permeate sample was collected in the middle of each $\mathrm{P}_{\mathrm{i}} / \mathrm{R}_{j}$ combination for $\mathrm{O} / \mathrm{G}$ analysis. Samples 
were preserved with one drop of concentrated sulfuric acid $\left(\mathrm{H}_{2} \mathrm{SO}_{4}\right)$, and stored at $45^{\circ} \mathrm{F}$ until the analysis was performed. Results from $\mathrm{O} / \mathrm{G}$ analysis are presented in Chapter 4.

Permeate Total Organic Carbon: Permeate total organic carbon (TOC) was determined using Standard Methods 505 A, Combustion-Infrared Method. One 10ml permeate sample was collected in the middle of each $\mathrm{P}_{i} / \mathrm{R}_{j}$ combination for TOC analysis. Samples were stored at $45^{\circ} \mathrm{F}$ until the analysis was performed. Results from TOC analysis are presented in Chapter 4.

Quality Assurance/Quality Control

Analytical triplicates were performed for each of the TOC samples. Sampling triplicates and analytical recoveries were performed on $10 \%$ of the TOC and $\mathrm{O} / \mathrm{G}$ samples. The membrane resistance was calculated after each membrane cleaning to measure the membrane's condition throughout the study. 


\section{CHAPTER 4.0 \\ RESULTS}

In this chapter, data from the permeate water quality experiments will be presented. The results of individual constant rotation/metal-working fluid concentration experiments (Oil and Grease and Total Organic Carbon) will be presented separately.

\section{$5 \%$ MW Fluid-1750 rpm}

The system was operated over an applied pressure range of 25 to $75 \mathrm{psig}$. The average permeate backpressure was 11.1 psi for a membrane rotational speed of 1750 rpm. Permeate oil and grease and total organic carbon versus average transmembrane pressure for the 5\% MW fluid-1750 rpm experiment are presented in Figure (4.1). Oil and grease varied greatly with transmembrane pressure, no definitive trends can be observed. Total organic carbon also varied greatly with transmembrane pressure, no definitive trend can be observed.

The membrane was cleaned using the standardized cleaning procedure discussed in Chapter 3. When the vessel was opened, a light sheen of oil was observed on the membrane surface. Thus, the membrane was hand-cleaned with dilute $(\sim 1 \%) \mathrm{H}_{2} \mathrm{SO}_{4}$. The

membrane and system were then re-cleaned using the standardized cleaning procedure and additional visual observations of the membrane's condition were made. No oil was visible on the membrane surface after the second membrane cleaning. The $\mathrm{pH}$ and conductivity of the distilled water used to determine the membrane resistance after the second cleaning were 6.92 and $6.29 \mu \mathrm{S} / \mathrm{cm}$, respectively. The resistance of the membrane was $1.13 \times 10^{12} \mathrm{~m}^{-1}$. 


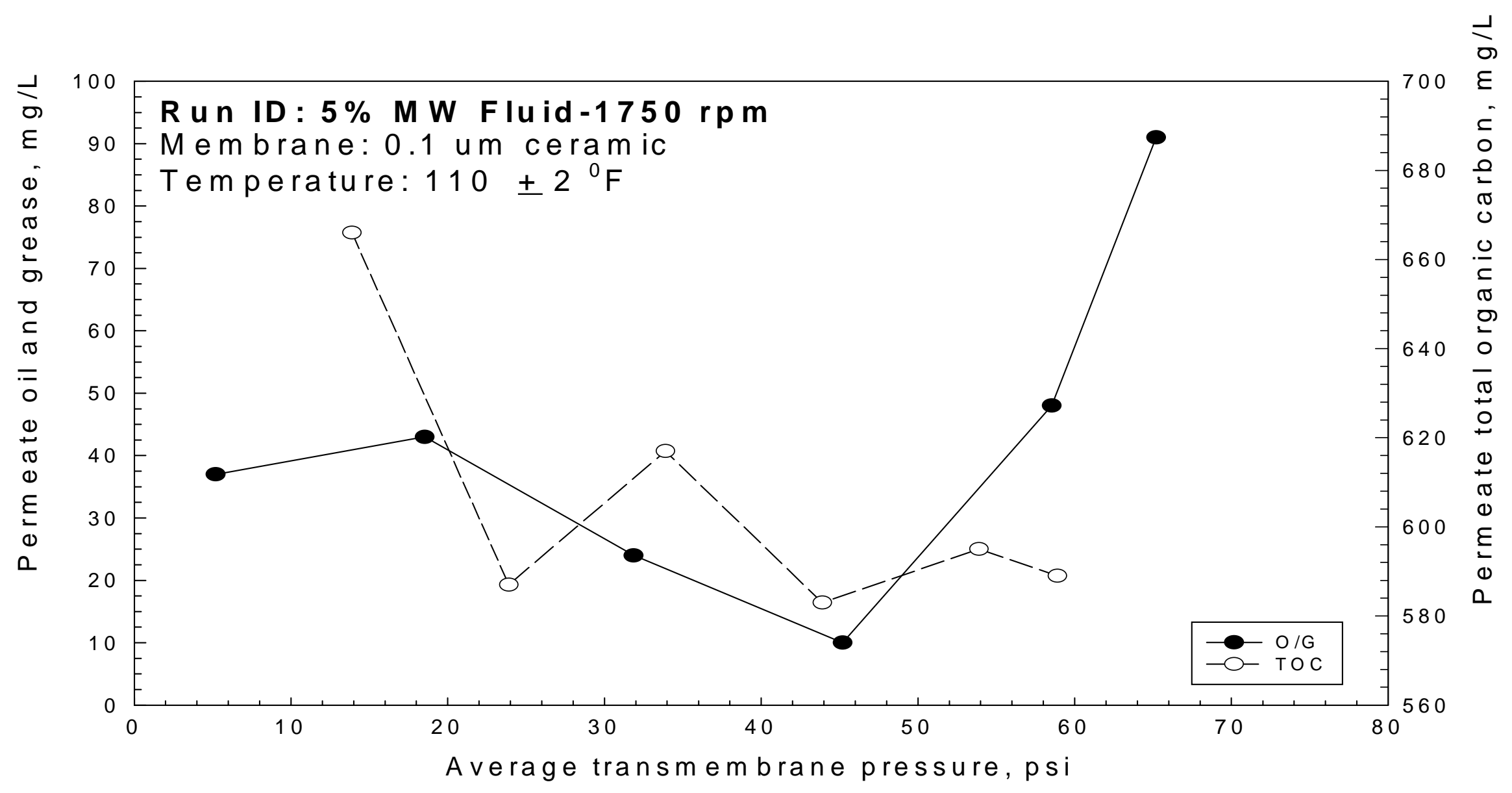

Figure (4.1)—Permeate Oil and Grease and Total Organic Carbon Versus Average Transmembrane Pressure For the 5\%-1750 rpm Combination. 


\section{$5 \%$ MW Fluid-1150 rpm}

The system was operated over an applied pressure range of 25 to $75 \mathrm{psig}$. The average permeate backpressure was 4.8 psi for a membrane rotational speed of $1150 \mathrm{rpm}$. Permeate oil and grease and total organic carbon versus average transmembrane pressure for the 5\% MW fluid-1150 rpm experiment are presented in Figure (4.2). Oil and grease varied greatly with increasing transmembrane pressure, no definitive trends can be observed. TOC decreased with increasing transmembrane pressure for each of the examined conditions.

The membrane was cleaned using the standardized cleaning procedure discussed in Chapter 3. No oil was visible on the membrane surface after cleaning. The $\mathrm{pH}$ and conductivity of the distilled water used to determine the membrane resistance after cleaning were 7.27 and $3.79 \mu \mathrm{S} / \mathrm{cm}$, respectively. The resistance of the membrane was $1.13 \times 10^{12} \mathrm{~m}^{-1}$

10\% MW Fluid-1750 rpm

The system was operated over an applied pressure range of 25 to $75 \mathrm{psig}$. The average permeate backpressure was 11.1 psi for a membrane rotational speed of 1750 rpm. Permeate oil and grease and total organic carbon versus average transmembrane pressure for the 10\% MW fluid-1750 rpm experiment are presented in Figure (4.3). In general, oil and grease decreased with increasing transmembrane pressure. However, total organic carbon increased with increasing transmembrane pressure.

The membrane was cleaned using the standardized cleaning procedure discussed in Chapter 3. No oil was visible on the membrane surface after cleaning. The $\mathrm{pH}$ and conductivity of the distilled water used to determine the membrane resistance 


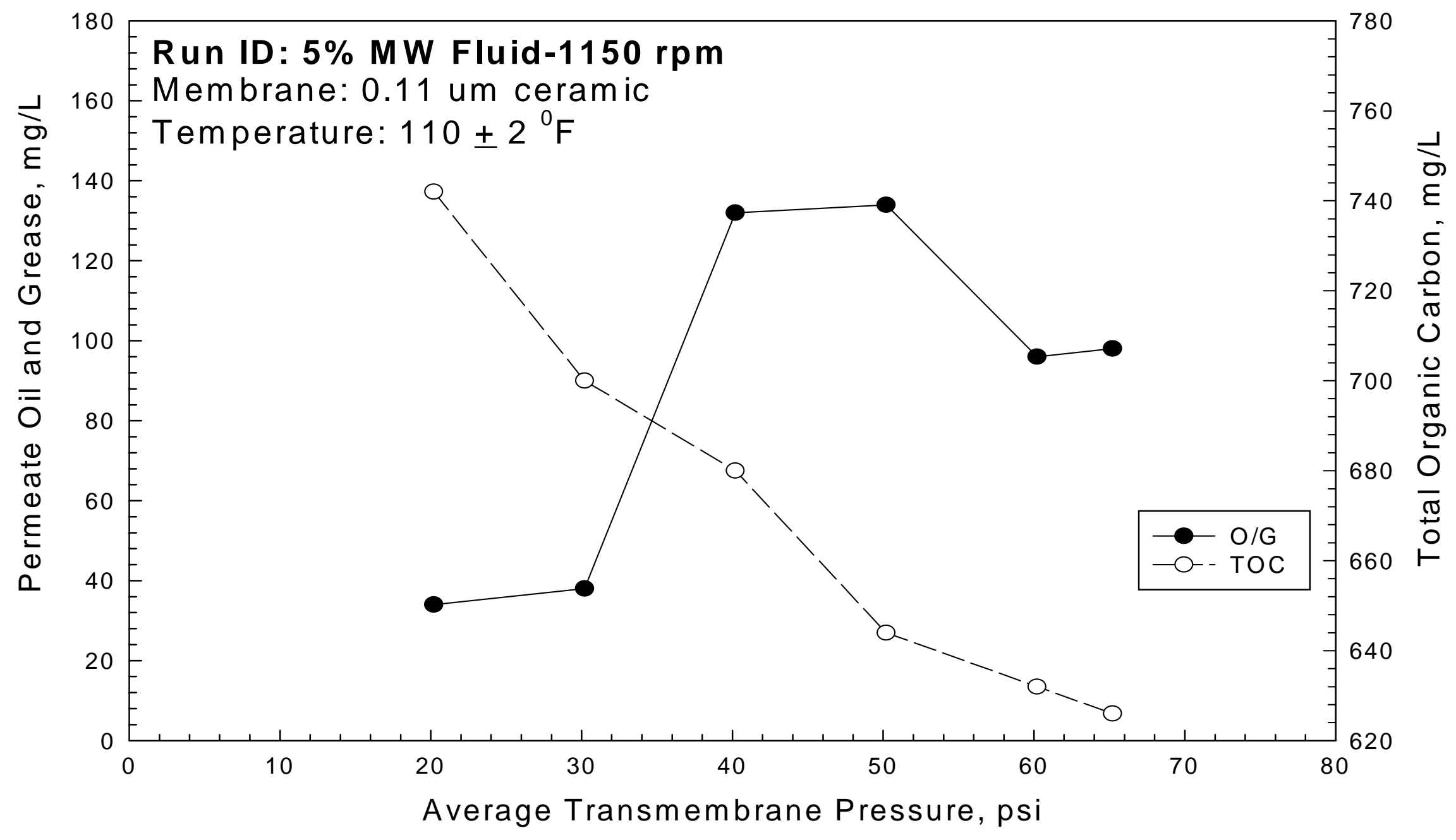

Figure (4.2)—Permeate Oil and Grease and Total Organic Carbon Versus Average Transmembrane Pressure For the 5\% MWF-1150 rpm Combination. 


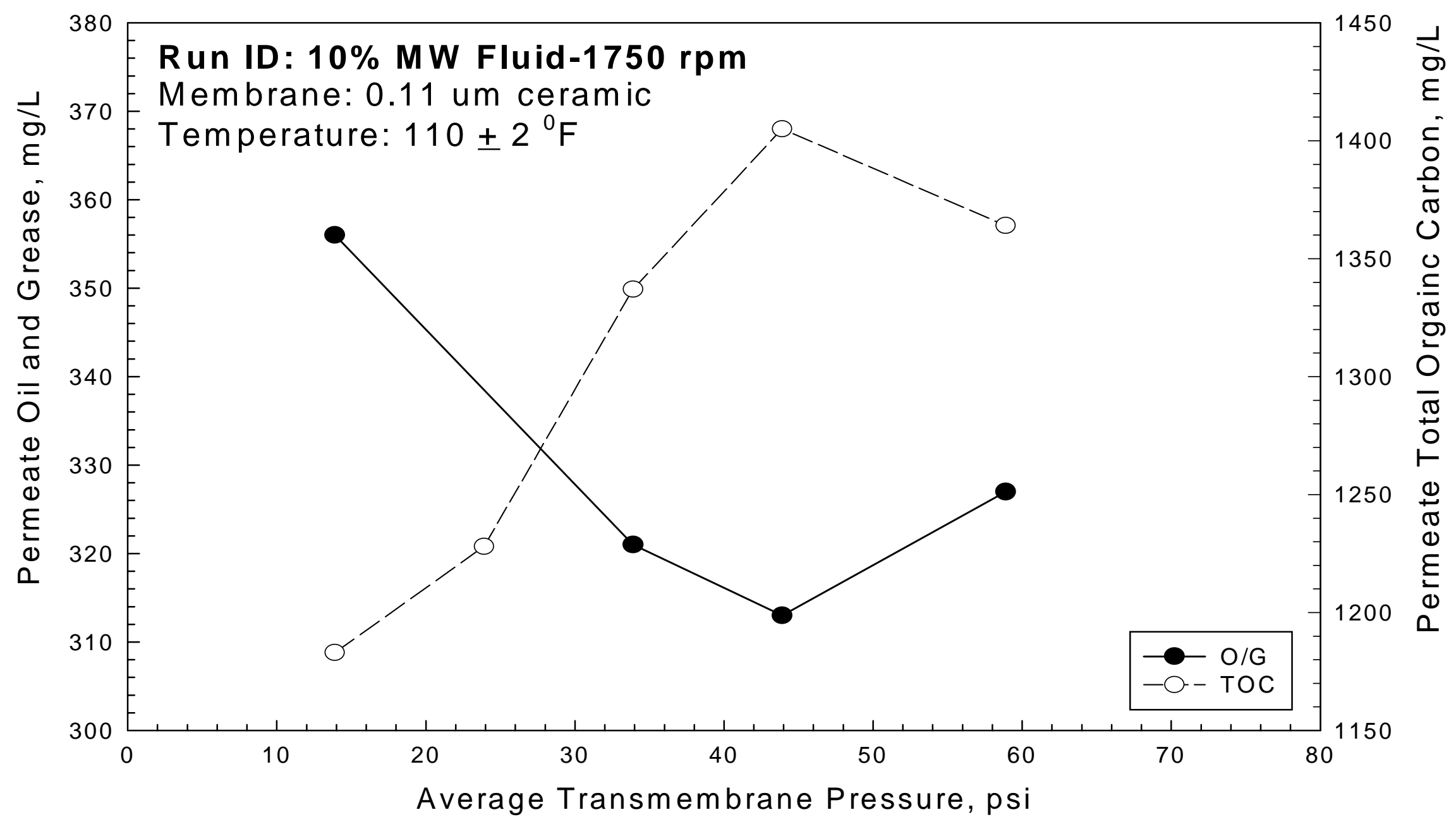

Figure (4.3)--Permeate Oil and Grease and Total Organic Carbon Versus Average Transmembrane For the $10 \%$ MWF-1750 rpm Combination. 
after cleaning were 7.25 and $5.15 \mu \mathrm{S} / \mathrm{cm}$, respectively. The resistance of the membrane was $2.22 \times 10^{12} \mathrm{~m}^{-1}$.

\section{$10 \%$ MW Fluid-1150 rpm}

The system was operated over an applied pressure range of 25 to $65 \mathrm{psig}$. The average permeate backpressure was 4.8 psi for a membrane rotational speed of 1150 rpm. Due to operational problems with the feed pump, it was not possible to maintain steady applied pressures at 45 and 75 psig. Permeate oil and grease and total organic carbon versus average transmembrane pressure for the $10 \% \mathrm{MW}$ fluid-1150 rpm experiment are presented in Figure (4.3). Oil and grease varied greatly with increasing transmembrane pressure, no definitive trends can be observed. Total organic carbon decreased with increasing transmembrane pressure between each applied pressure condition.

The membrane was cleaned using the standardized cleaning procedure discussed in Chapter 3. No oil was visible on the membrane surface after cleaning. The $\mathrm{pH}$ and conductivity of the distilled water used to determine the membrane resistance after cleaning were 7.15 and $4.04 \mu \mathrm{S} / \mathrm{cm}$, respectively. The resistance of the membrane was $1.51 \times 10^{12} \mathrm{~m}^{-1}$.

\section{$15 \%$ MW Fluid-1750 rpm}

The system was operated over an applied pressure range of 20 to $70 \mathrm{psig}$. The average permeate backpressure was 11.1 for a membrane rotational speed of 1750 rpm. Permeate oil and grease and total organic carbon versus average transmembrane pressure for the 15\% MW fluid-1750 rpm experiment are presented in Figure (4.4). Oil and grease varied greatly with increasing transmembrane pressure, no definitive 
trends can be observed. Total organic carbon varied greatly with increasing transmembrane pressure, no definitive trends can be observed.

The membrane was cleaned using the standardized cleaning procedure discussed in Chapter 3. No oil was visible on the membrane surface after cleaning. The $\mathrm{pH}$ and conductivity of the distilled water used to determine the membrane resistance after cleaning were 6.93 and $3.84 \mu \mathrm{S} / \mathrm{cm}$, respectively. The resistance of the membrane was $1.76 \times 10^{12} \mathrm{~m}^{-1}$.

\section{5\% MW Fluid-1150 rpm}

The system was operated over an applied pressure range of 15 to $70 \mathrm{psig}$. The average permeate backpressure was 4.8 psi for a membrane rotational speed of 1150 rpm. Permeate oil and grease and total organic carbon versus average transmembrane pressure for the $15 \% \mathrm{MW}$ fluid-1150 rpm experiment are presented in Figure (4.6). In general, oil and grease decreased with increasing transmembrane pressure. Similarly, total organic carbon decreased with increasing transmembrane pressure.

The membrane was cleaned using the standardized cleaning procedure discussed in Chapter 3. No oil was visible on the membrane surface after cleaning. The $\mathrm{pH}$ and conductivity of the distilled water used to determine the membrane resistance after cleaning were 6.98 and $3.98 \mu \mathrm{S} / \mathrm{cm}$, respectively. The resistance of the membrane was $1.85 \times 10^{12} \mathrm{~m}^{-1}$.

\section{$20 \%$ MW Fluid-1750 rpm}

The system was operated over an applied pressure range of 20 to $70 \mathrm{psig}$. The average permeate backpressure was 11.1 psi for a membrane rotational speed of 1750 


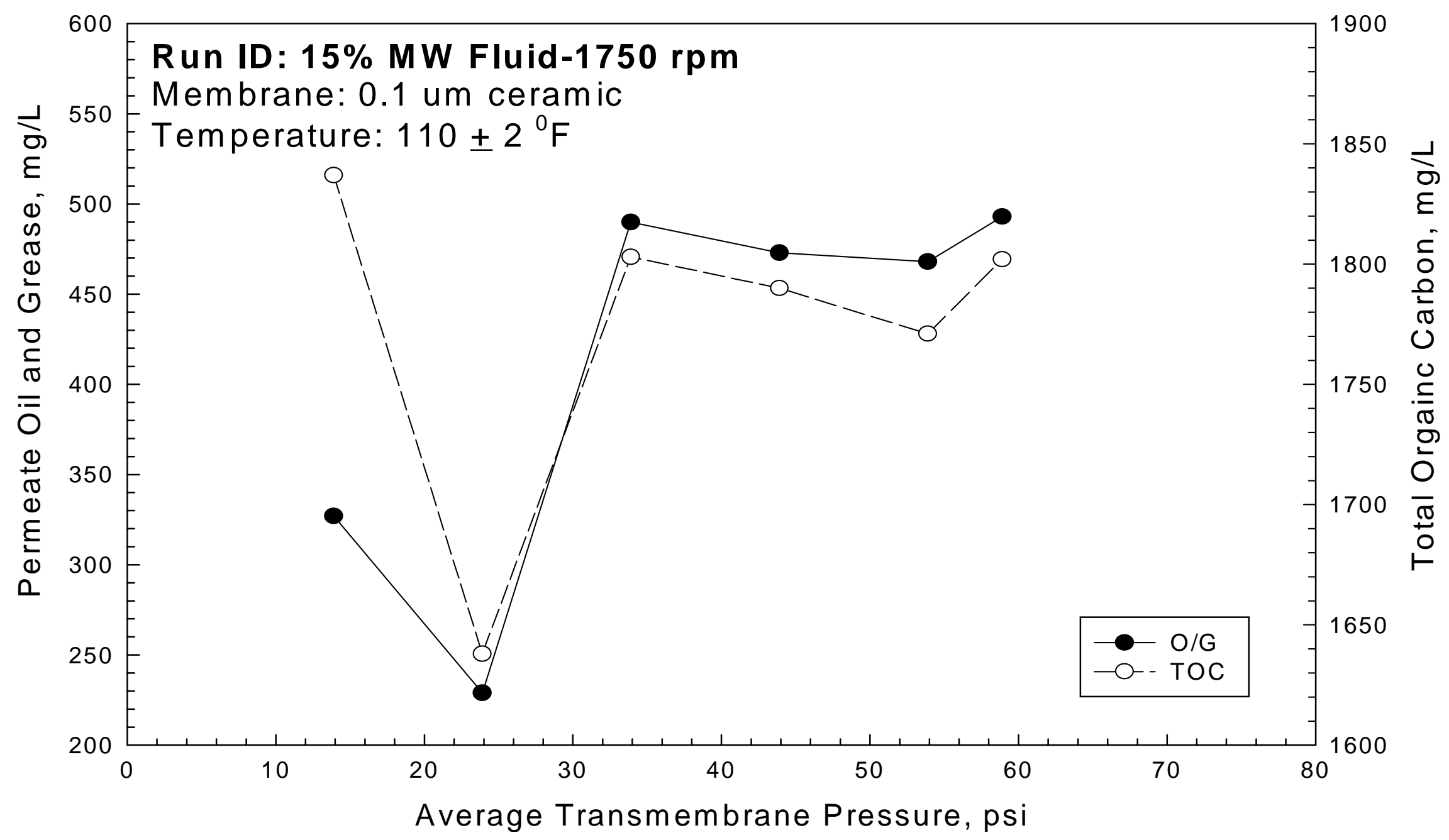

Figure (4.4)_Permeate Oil and Grease and Total Organic Carbon Versus Average Transmembrane Pressure for the 15\% MWF-1750 rpm Combination. 


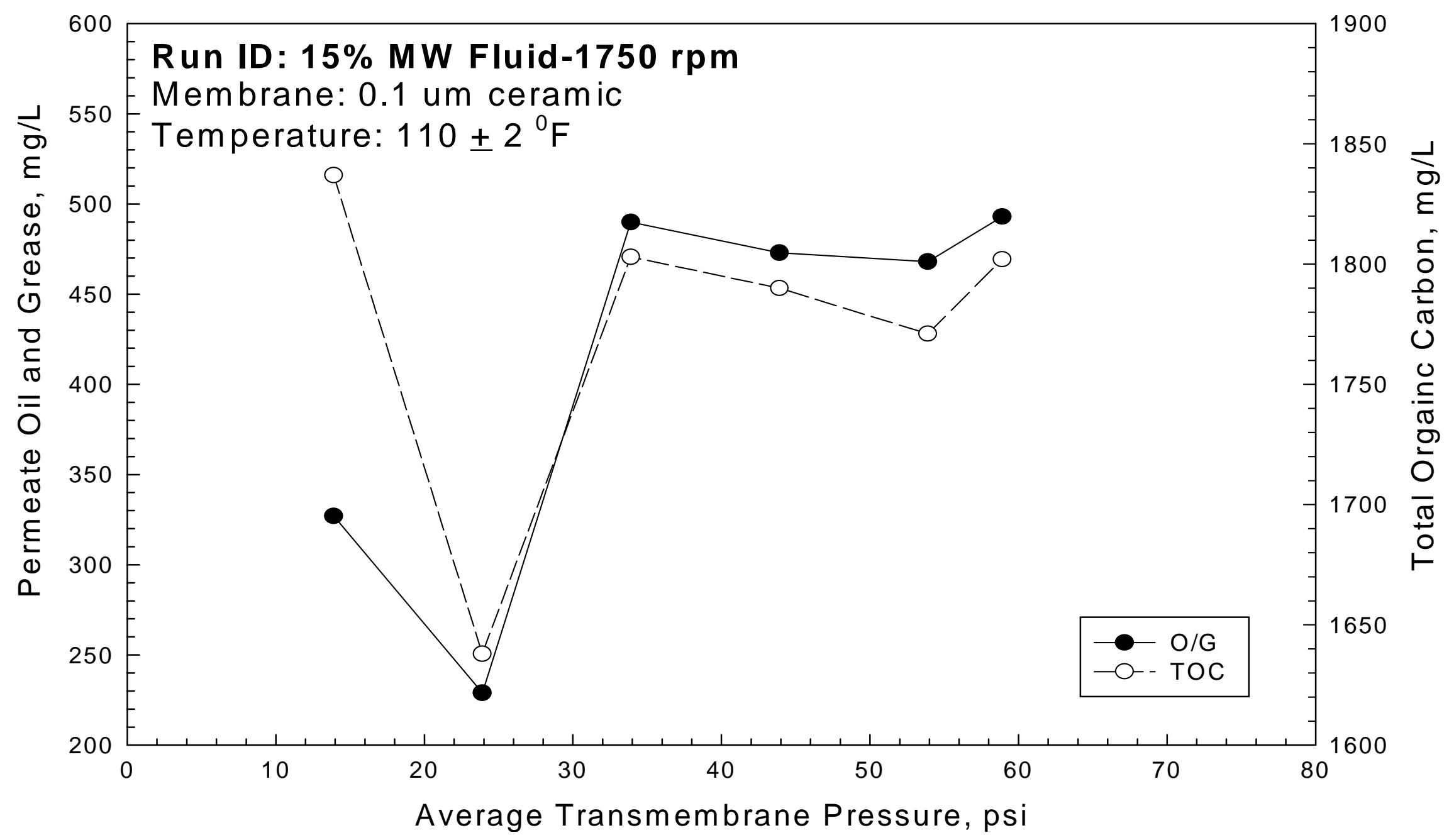

Figure (4.5)—Permeate Oil and Grease and Total Organic Carbon Versus Average Transmembrane Pressure For the 15\% MWF-1750 rpm Combination. 


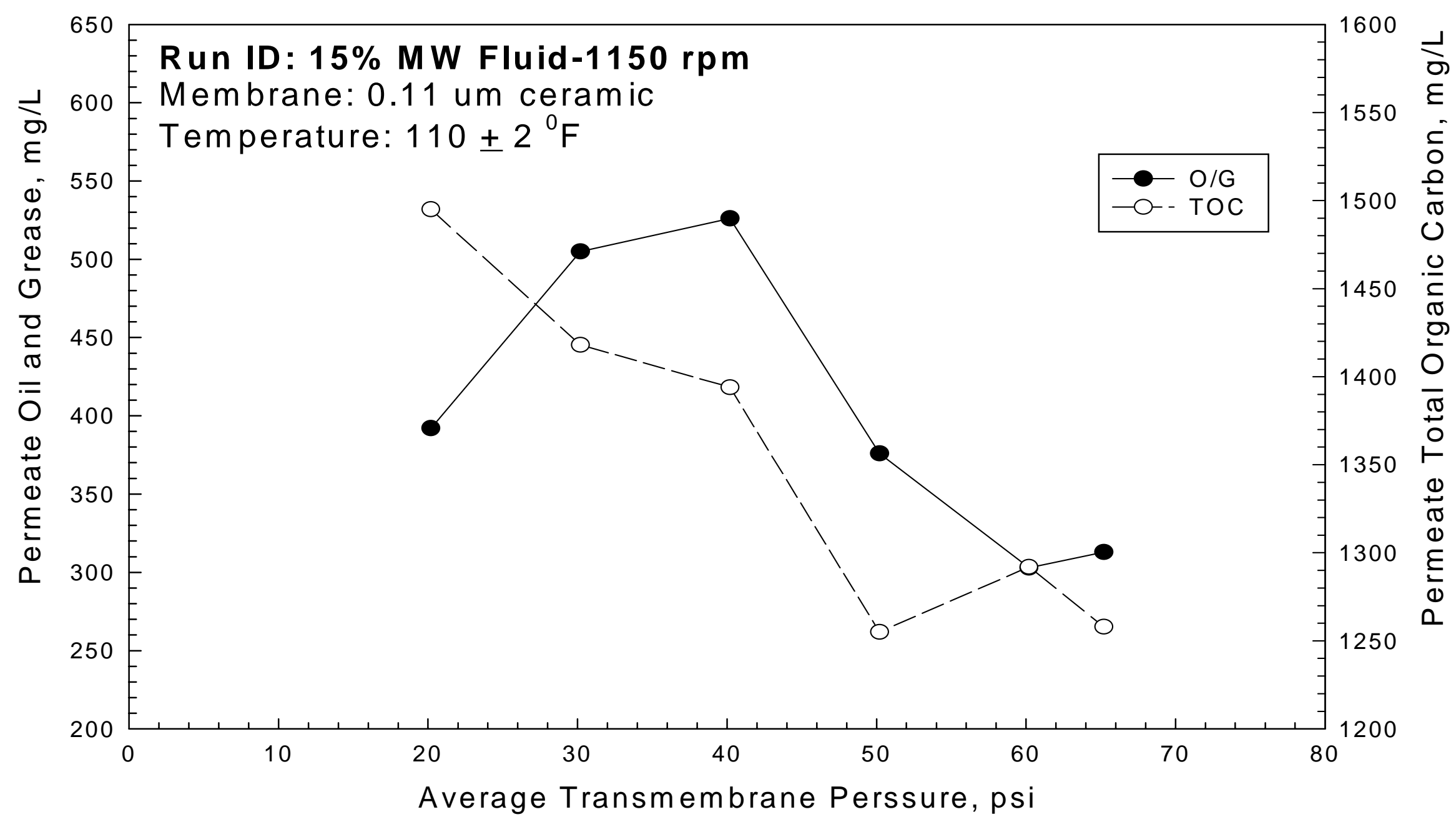

Figure (4.6)—Permeate Oil and Grease and Total Organic Carbon Versus Average Transmembrane Pressure For the 15\% MWF-1150 rpm Combination. 
rpm. Permeate oil and grease and total organic carbon versus average transmembrane pressure for the 20\% MW fluid-1750 rpm experiment are presented in Figure (4.7). Oil and grease varied greatly with increasing transmembrane pressure, no definitive trends can be observed. Similarly, total organic carbon varied greatly with increasing transmembrane pressure, no definitive trends can be observed.

The membrane was cleaned using the standardized cleaning procedure discussed in Chapter 3. No oil was visible on the membrane surface after cleaning. The $\mathrm{pH}$ and conductivity of the distilled water used to determine the membrane resistance after cleaning were 6.87 and $2.91 \mu \mathrm{S} / \mathrm{cm}$, respectively. The resistance of the membrane was $1.64 \times 10^{12} \mathrm{~m}^{-1}$.

\section{$20 \%$ MW Fluid-1150 rpm}

The system was operated over an applied pressure range of 15 to $70 \mathrm{psig}$. The average permeate backpressure was 4.8 psi for a membrane rotational speed of $1150 \mathrm{rpm}$. Permeate oil and grease and total organic carbon versus average transmembrane pressure for the $20 \%$ MW fluid-1150 rpm experiment are presented in Figure (4.8). In general, oil and grease decreased with increasing transmembrane pressure. However, total organic carbon varied greatly with increasing transmembrane pressure, no definitive trends can be observed.

The membrane was cleaned using the standardized cleaning procedure discussed in Chapter 3. No oil was visible on the membrane surface after cleaning. The $\mathrm{pH}$ and conductivity of the distilled water used to determine the membrane resistance after cleaning were 6.73 and $4.14 \mu \mathrm{S} / \mathrm{cm}$, respectively. The resistance of the membrane was $3.12 \times 10^{12} \mathrm{~m}^{-1}$ 


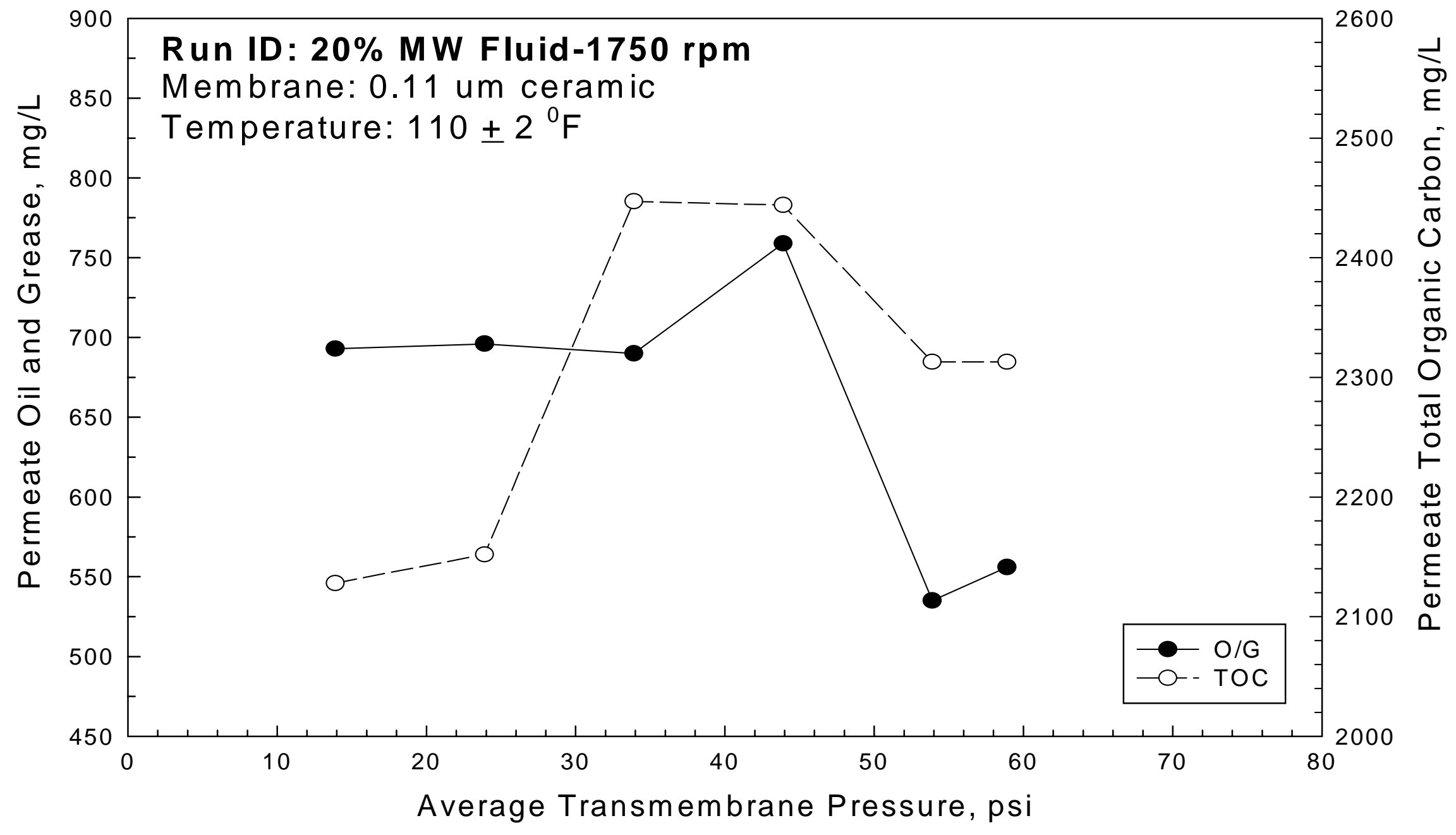

Figure (4.7)—Permeate Oil and Grease and Total Organic Carbon Versus Average Transmembrane Pressure For the 20\% MWF-1750 rpm Combination. 


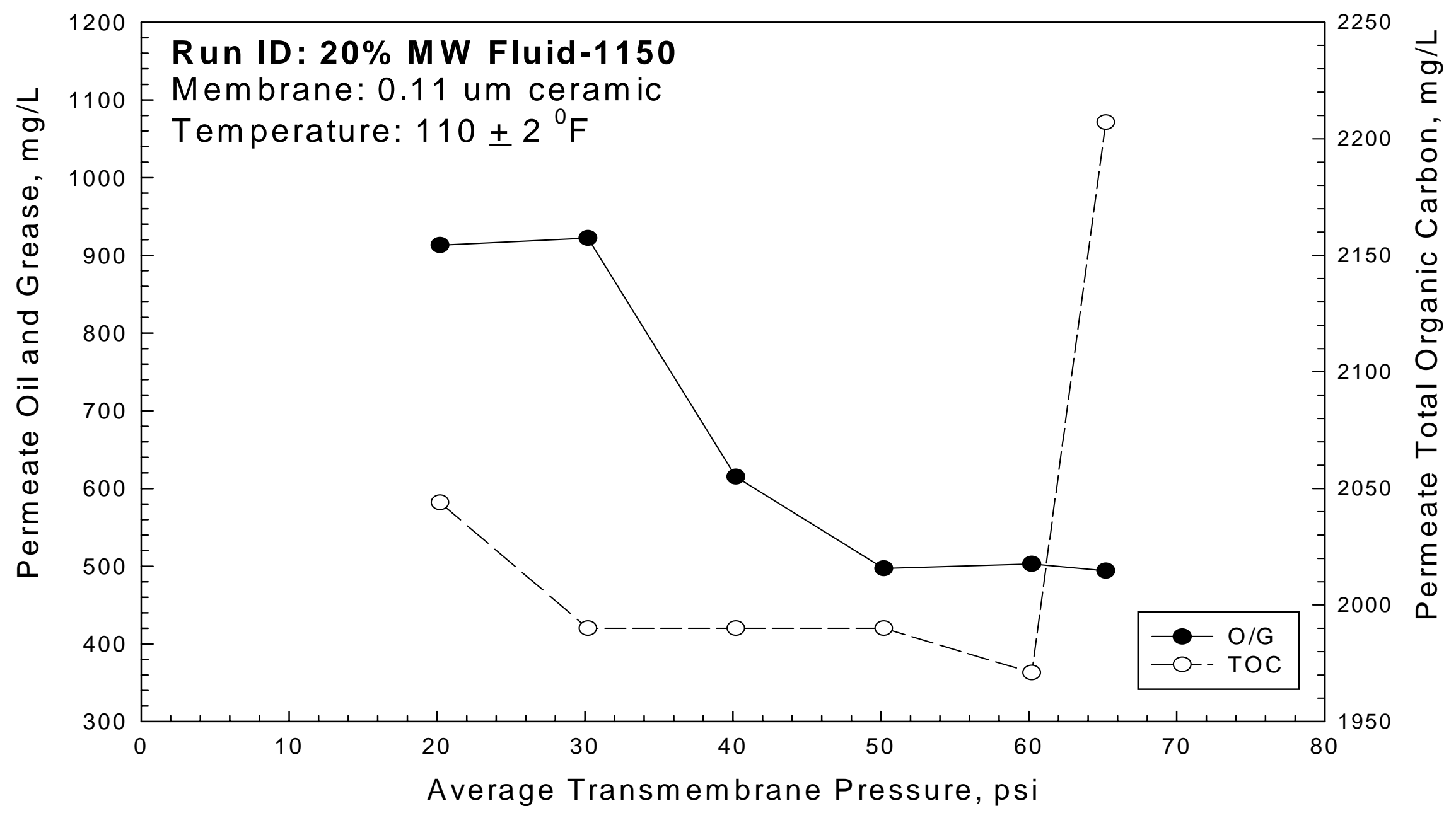

Figure (4.8)—Permeate Oil and Grease and Total Organic Carbon Versus Average Transmembrane Pressure For the 20\% MWF-1150 rpm Combination. 


\section{$30 \%$ MW Fluid-1750 rpm}

The system was operated over an applied pressure range of 25 to $70 \mathrm{psig}$. The average permeate backpressure was 11.1 psi for a membrane rotational speed of 1750 rpm. Permeate oil and grease and total organic carbon versus average transmembrane pressure for the $30 \% \mathrm{MW}$ fluid-1750 rpm experiment are presented in Figure (4.9). Oil and grease varied greatly with increasing transmembrane pressure, no definitive trends can be observed. Total organic carbon also varied greatly with increasing transmembrane pressure, no definitive trends can be observed.

The membrane was cleaned using the standardized cleaning procedure discussed in Chapter 3. No oil was visible on the membrane surface after cleaning. The $\mathrm{pH}$ and conductivity of the distilled water used to determine the membrane resistance after cleaning were 6.93 and $3.83 \mu \mathrm{S} / \mathrm{cm}$, respectively. The resistance of the membrane was $2.42 \times 10^{12} \mathrm{~m}^{-1}$

\section{$30 \%$ MW Fluid-1150 rpm}

The system was operated over an applied pressure range of 15 to $70 \mathrm{psig}$. The average permeate backpressure was 4.8 psi for a membrane rotational speed of $1150 \mathrm{rpm}$. Permeate oil and grease and total organic carbon versus average transmembrane for the 30\% MW fluid-1150 rpm experiment are presented in Figure (4.10). In general, oil and grease increased with increasing transmembrane pressure. However, total organic carbon decreased with increasing transmembrane pressure.

The membrane was cleaned using the standardized cleaning procedure discussed in Chapter 3. No oil was visible on the membrane surface after cleaning. The $\mathrm{pH}$ and conductivity of the distilled water used to determine the membrane resistance after 


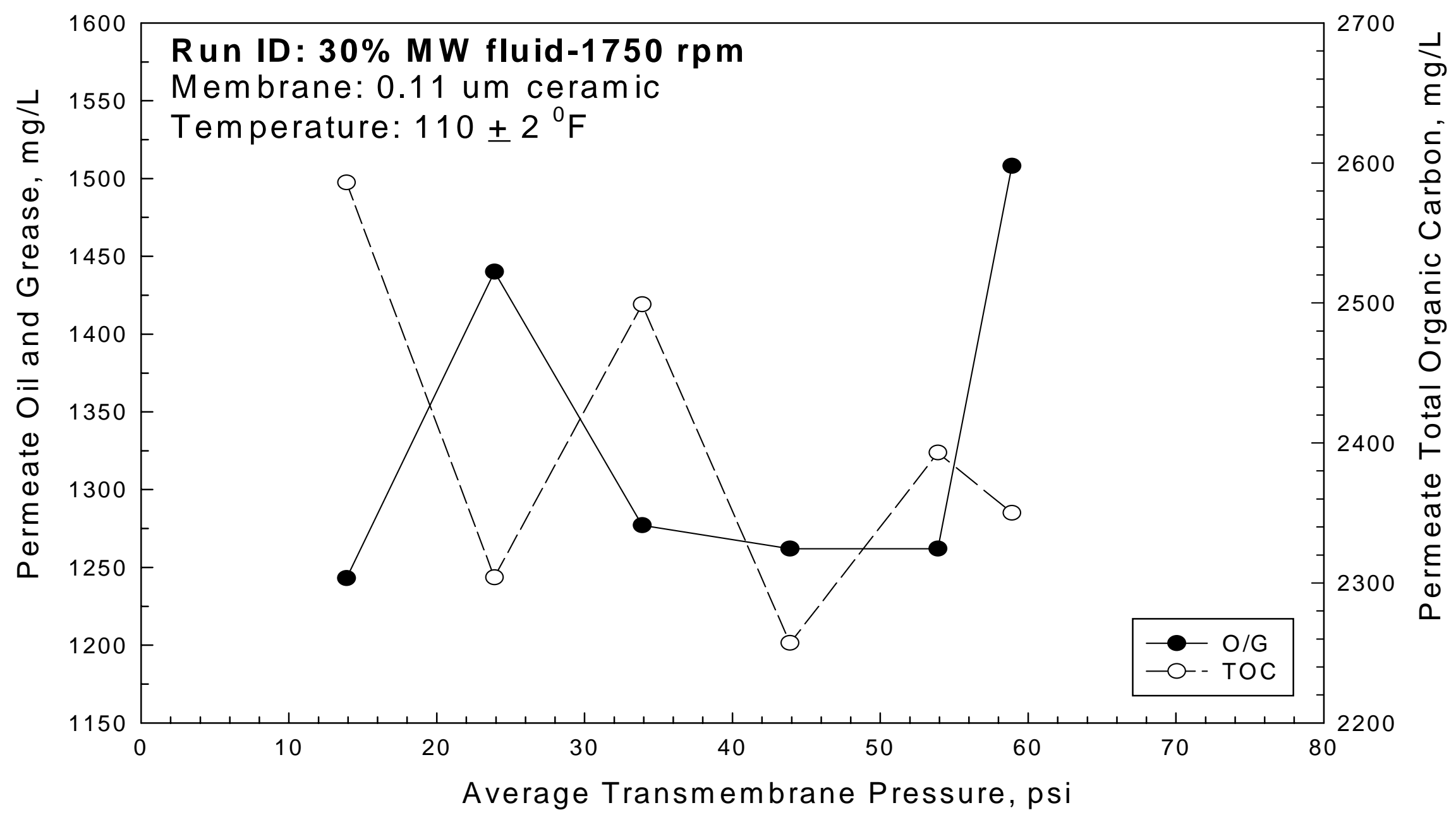

Figure (4.9)—Permeate Oil and Grease and Total Organic Carbon Versus Average Transmembrane Pressure For the $30 \%$ MWF-1750 rpm Combination. 


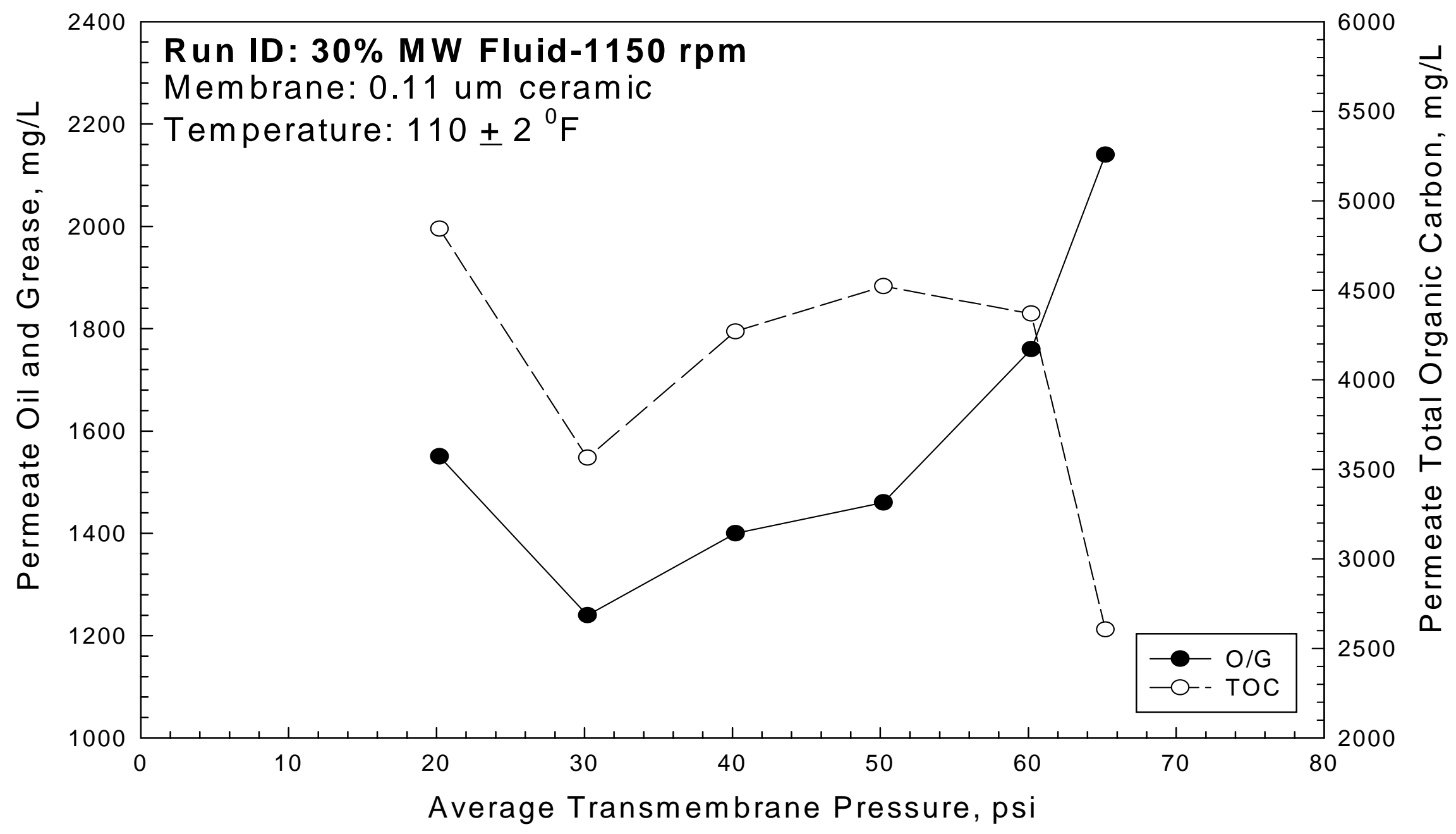

Figure (4.10)_Permeate Oil and Grease and Total Organic Carbon Versus Average Transmembrane Pressure For the $30 \%$ MWF-1150 rpm Combination. 
cleaning were 6.69 and $3.04 \mu \mathrm{S} / \mathrm{cm}$, respectively. The resistance of the membrane was $3.06 \times 10^{12} \mathrm{~m}^{-1}$

\section{$40 \%$ MW Fluid-1750 rpm}

The system was operated over an applied pressure range of 25 to $70 \mathrm{psig}$. The average permeate backpressure was 11.1 psi for a membrane rotational speed of 1750 rpm. Permeate oil and grease and total organic carbon versus average transmembrane pressure for the $40 \%$ MW fluid-1750 rpm experiment are presented in Figure (4.11). In general, oil and grease and total organic carbon both increased with increasing transmembrane pressure.

The membrane was cleaned using the standardized cleaning procedure discussed in Chapter 3. No oil was visible on the membrane surface after cleaning. The $\mathrm{pH}$ and conductivity of the distilled water used to determine the membrane resistance after cleaning were 6.80 and $2.84 \mu \mathrm{S} / \mathrm{cm}$, respectively. The resistance of the membrane was $1.57 \times 10^{12} \mathrm{~m}^{-1}$

\section{0\% MW Fluid-1150 rpm}

The system was operated over an applied pressure range of 15 to $70 \mathrm{psig}$. The average permeate backpressure was 4.8 for a membrane rotational speed of $1150 \mathrm{rpm}$. Permeate oil and grease and total organic carbon versus average transmembrane for the 40\% MW fluid-1150 rpm experiment are presented in Figure (4.12). In general, oil and grease and total organic carbon both increased with increasing transmembrane pressure.

The membrane was cleaned using the standardized cleaning procedure discussed

in Chapter 3. No oil was visible on the membrane surface after cleaning. The $\mathrm{pH}$ and conductivity of the distilled water used to determine the membrane resistance after 


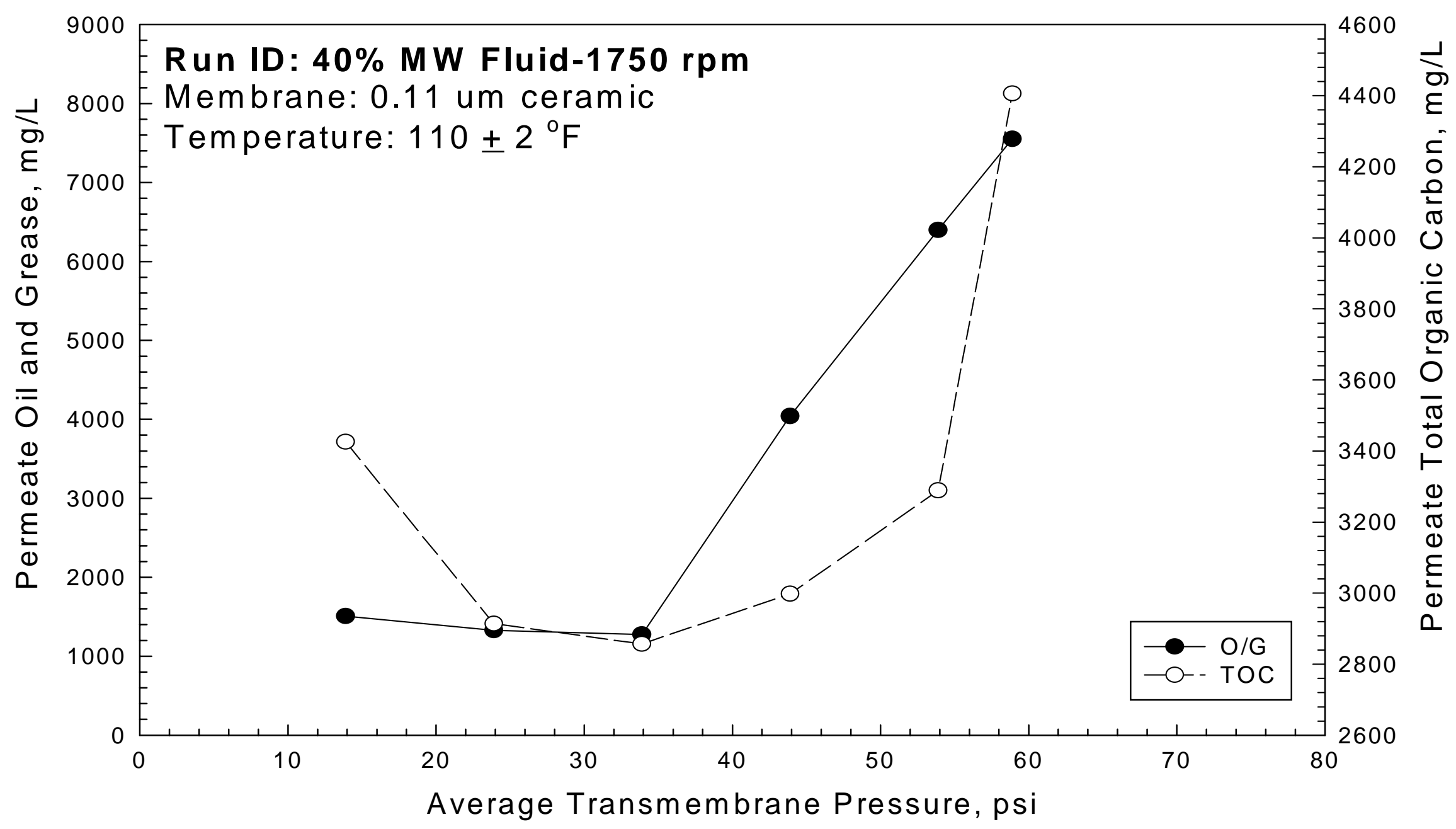

Figure (4.11)_Permeate Oil and Grease and Total Organic Carbon Versus Average Transmembrane Pressure For the 40\% MWF-1750 rpm Combination. 


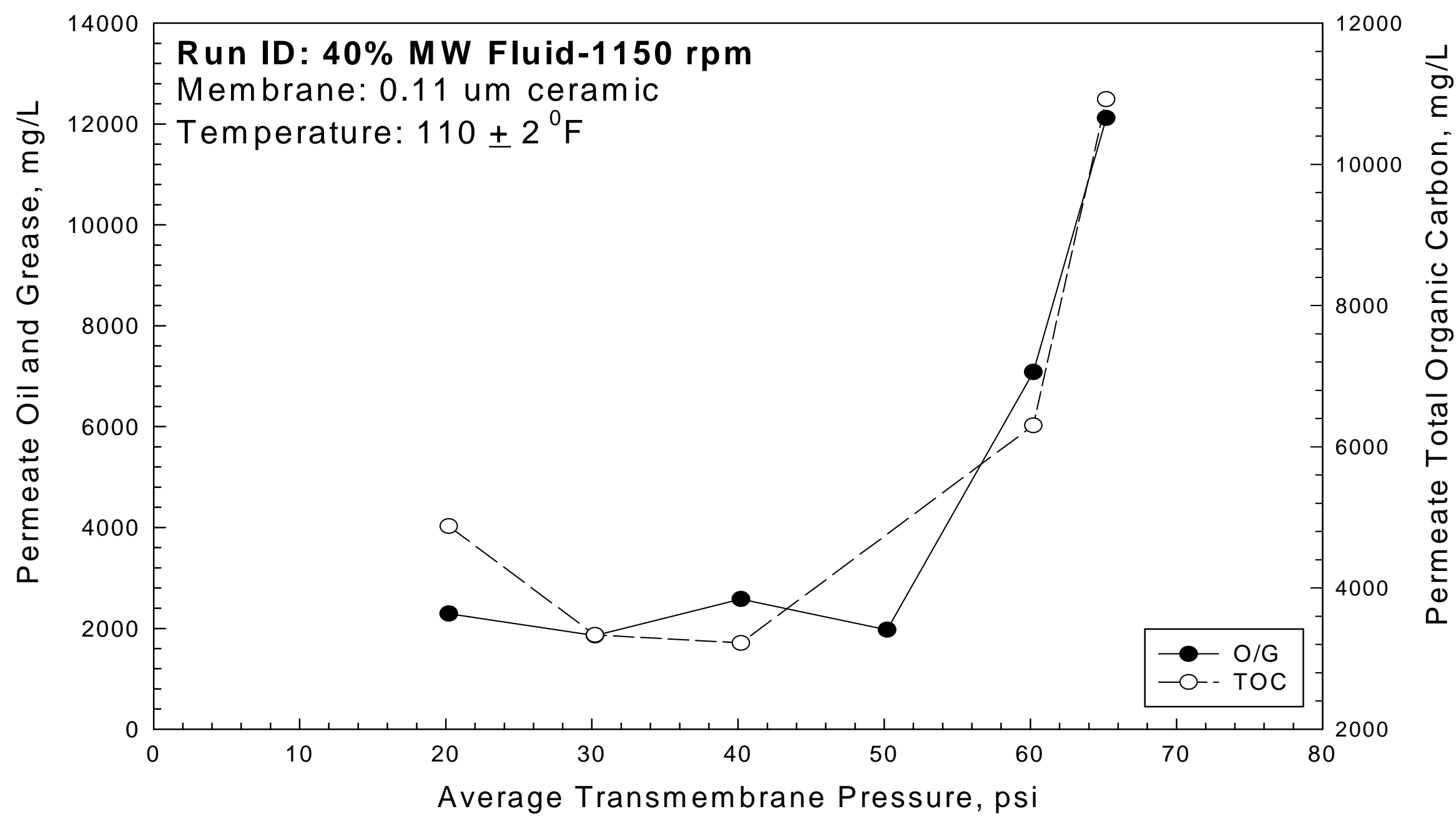

Figure (4.12)—Permeate Oil and Grease and Total Organic Carbon Versus Average Transmembrane Pressure For the 40\% MWF-1150 rpm Combination. 
cleaning were 6.73 and $4.14 \mu \mathrm{S} / \mathrm{cm}$, respectively. The resistance of the membrane was $1.72 \times 10^{12} \mathrm{~m}^{-1}$ 


\section{CHAPTER 5.0 \\ DISCUSSION}

Permeate oil and grease versus feed oil content, for the $1750 \mathrm{rpm}-25,55$ and 75 psi combinations is presented in Figure (5.1). Data presented in Figure (5.1) are representative of the broad range of experimental results obtained in this study. Increases in $\mathrm{O} / \mathrm{G}$ were observed with $\mathrm{OC}_{\mathrm{k}}$ for each of the $\mathrm{R}_{\mathrm{j}} / \mathrm{P}_{\mathrm{i}}$ combinations examined in this study. Reed, et al. (1997) also reported an increase in O/G with feed oil concentration. Permeate total organic carbon versus feed oil content, for the $1750 \mathrm{rpm}-35,65$ and $70 \mathrm{psi}$ combinations is presented in Figure (5.2). Data presented in Figure (5.2) are representative of the broad range of experimental results obtained in this study. Increases in TOC were observed with $\mathrm{OC}_{k}$ for each of the $\mathrm{R}_{\mathrm{j}} / \mathrm{P}_{\mathrm{i}}$ combinations examined in this study. Lipp et al. (1988) also reported increases in TOC with feed oil concentration. An increase in $\mathrm{OC}_{\mathrm{k}}$ resulted in an increased concentration of rejected oil droplets at the membrane surface (Cheryan 1997). It is hypothesized that the growing concentration of oil droplets at the membrane surface resulted in a higher probability of oil permeation through the membrane pores. Therefore, increases in both $\mathrm{O} / \mathrm{G}$ and TOC were observed. Also, Lipp et al. (1988) reported that the release of surfactants and other additives in the MW fluid during the coalescence of oil droplets in the growing concentration boundary layer was responsible for increased TOC.

Feed viscosity versus $\%$ metal working fluid is presented in Figure (5.3). The plots of both $\mathrm{O} / \mathrm{G}$ and TOC versus $\mathrm{OC}_{\mathrm{k}}$, presented in Figures (5.1) and (5.2), are similar to the relationship between feed absolute viscosity and $\mathrm{OC}_{\mathrm{k}}$ presented by Viadero (1997). 


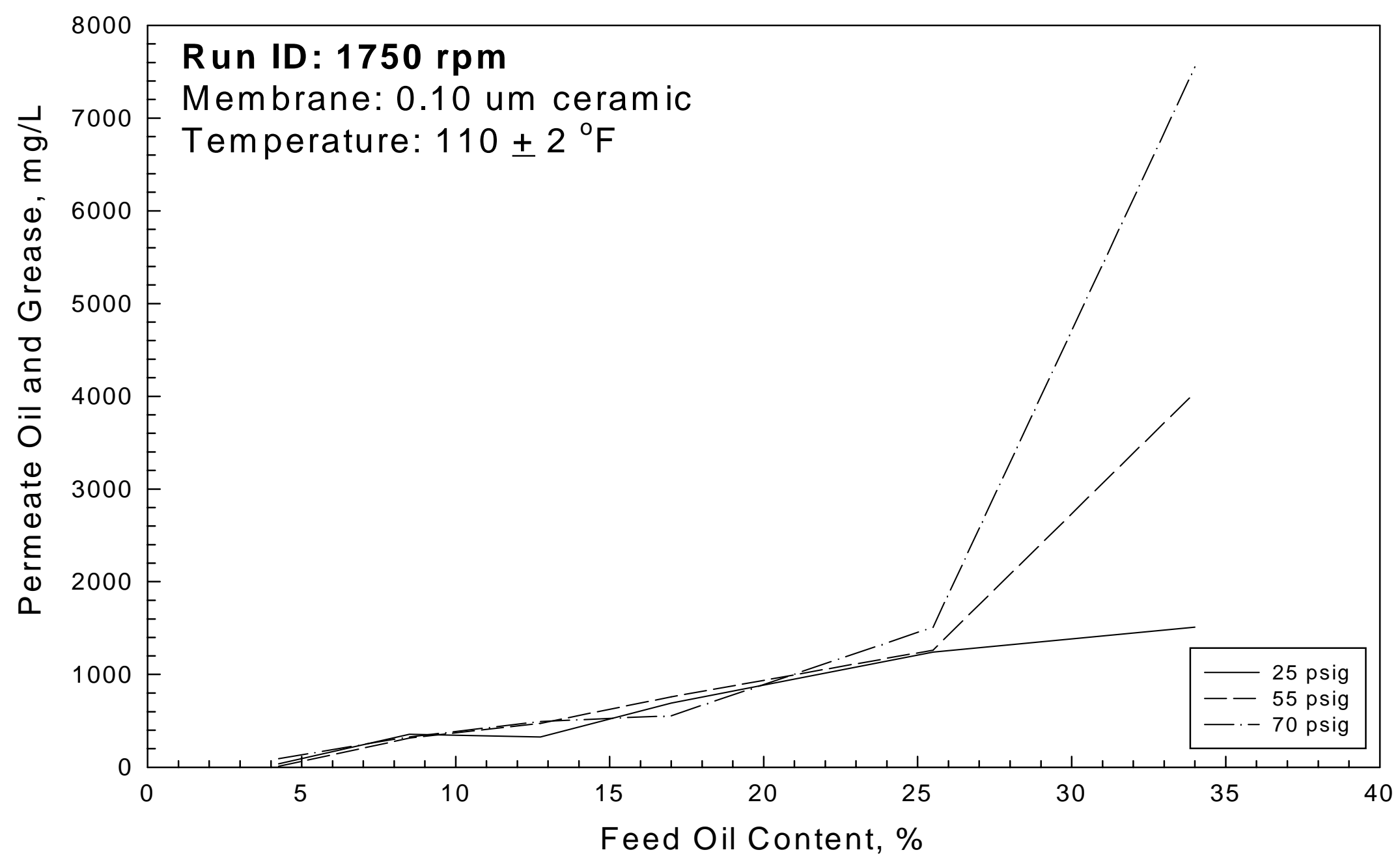

Figure (5.1)—Permeate Oil and Grease Versus Feed Oil Content For the 1750 rpm-25, 55 and 70 psig Combinations. 


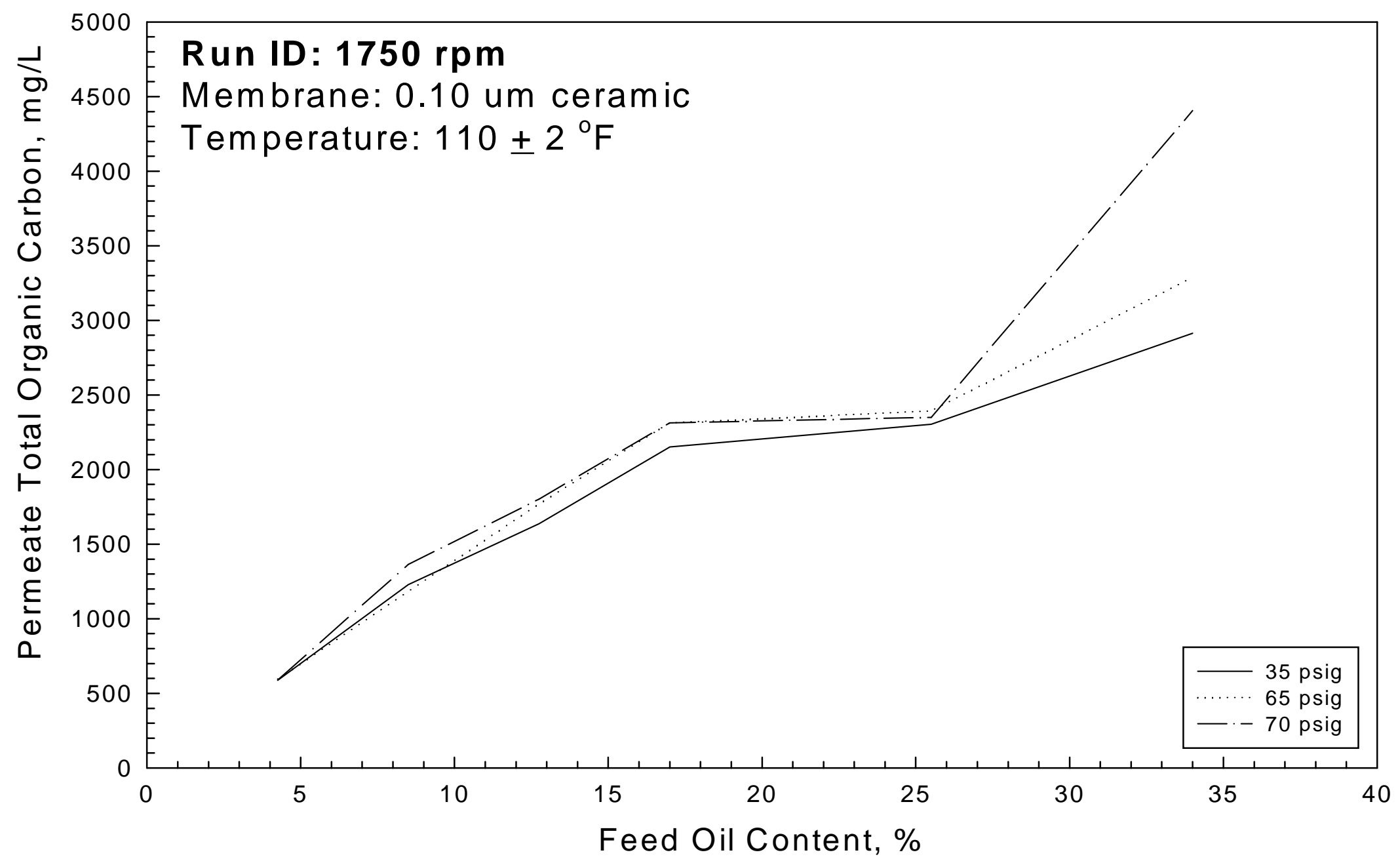

Figure (5.2)--Permeate Total Organic Carbon Versus Feed Oil Content For the 1750 rpm-35, 65 and 70 psig Combinations. 


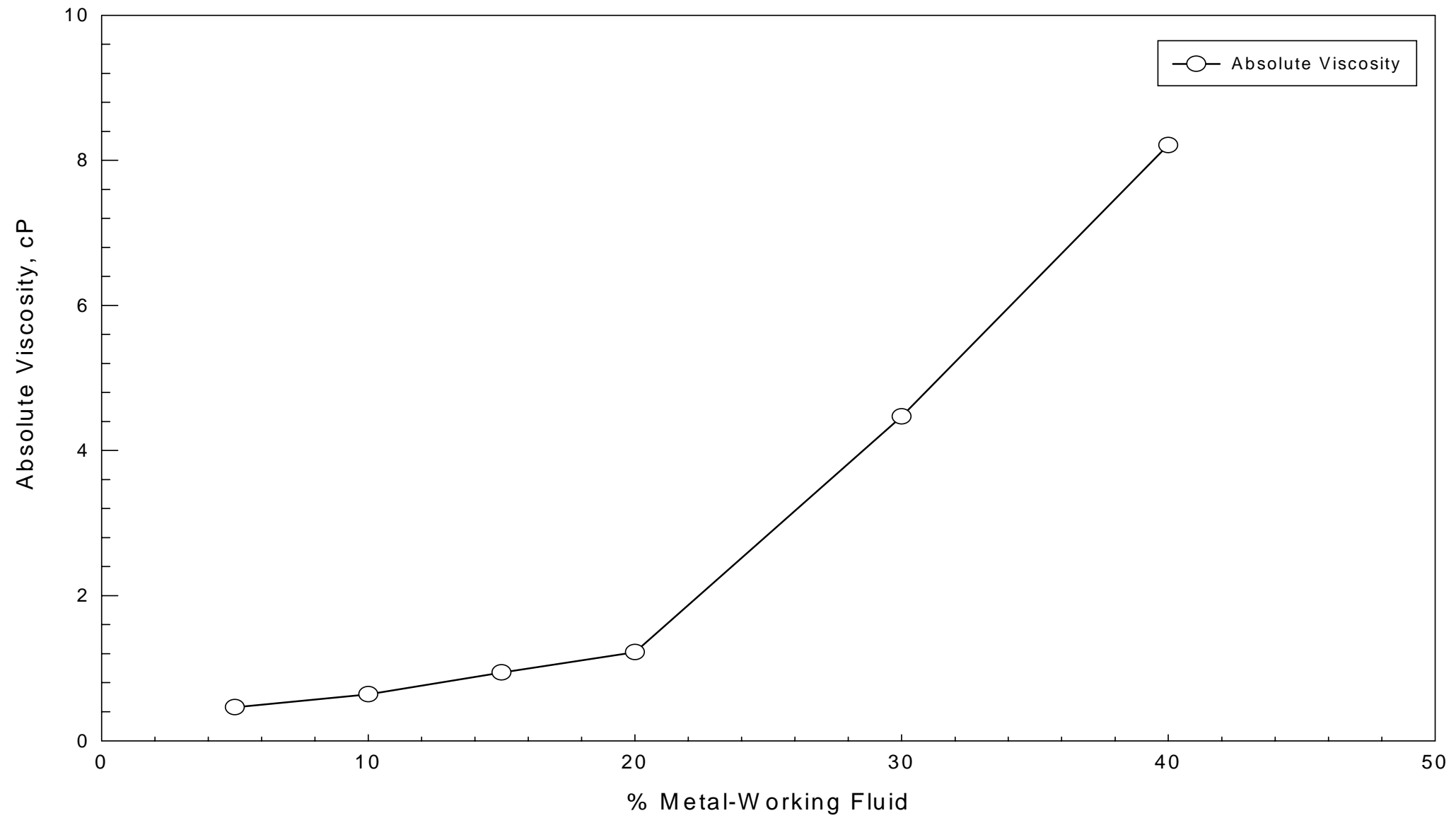

Figure (5.3)—MW Fluid Absolute Viscosity Versus \% Metal-Working Fluid (After Viadero (1997)). 
$\mathrm{O} / \mathrm{G}, \mathrm{TOC}$ and feed viscosity all increase versus $\mathrm{OC}_{\mathrm{k}}<25 \%$. At a concentration of 25$30 \%$ oil, the increase with $\mathrm{OC}_{\mathrm{k}}$ becomes considerably more substantial. It is hypothesized that the more substantial increases in O/G and TOC at 25-30\% oil can be attributed to the similar changes in the feed viscosity at the same oil concentrations. As viscosity increases, decreased turbulence will result in higher concentrations of rejected oil droplets at the membrane surface (Cheryan 1997).

Permeate oil and grease and total organic carbon versus average TMP for the $40 \%$ MW fluid combinations are presented in Figure (5.4). Permeate total organic carbon versus average TMP for the 40\% MW fluid combinations are presented in Figure (5.5). Data presented in Figures (5.4) and (5.5) are representative of the broad range of experimental results obtained in this study. In general, both O/G and TOC increased with TMP. The increase in TMP, resulted in a subsequent increase in convective transport of oil droplets to the membrane; therefore, augmenting the degree of polarization (Cheryan 1997). Nazzal and Wiesner (1996) reported a decrease in solute rejection with increased TMP. It is hypothesized that as TMP increased, the critical pressure required to force the entry of an oil droplet into a membrane pore was approached. Furthermore, the increased TMP and concentration of oil droplets at the membrane surface resulted in a greater probability of oil permeation through the membrane pores.

In general, both $\mathrm{O} / \mathrm{G}$ and TOC decreased with increasing $\mathrm{R}_{\mathrm{j}}$ as observed in Figures (5.4) and (5.5). Reed, et al. (1997) reported no adverse affects on O/G with variable $\mathrm{R}_{\mathrm{j}}$; similarly, Lipp et al. (1988) reported no change in TOC with variable $\mathrm{R}_{\mathrm{j}}$. Increasing $R_{j}$ resulted in greater hydraulic turbulence at the membrane surface (Viadero and Reed 1998), therefore decreasing the concentration of rejected oil droplets (Cheryan 


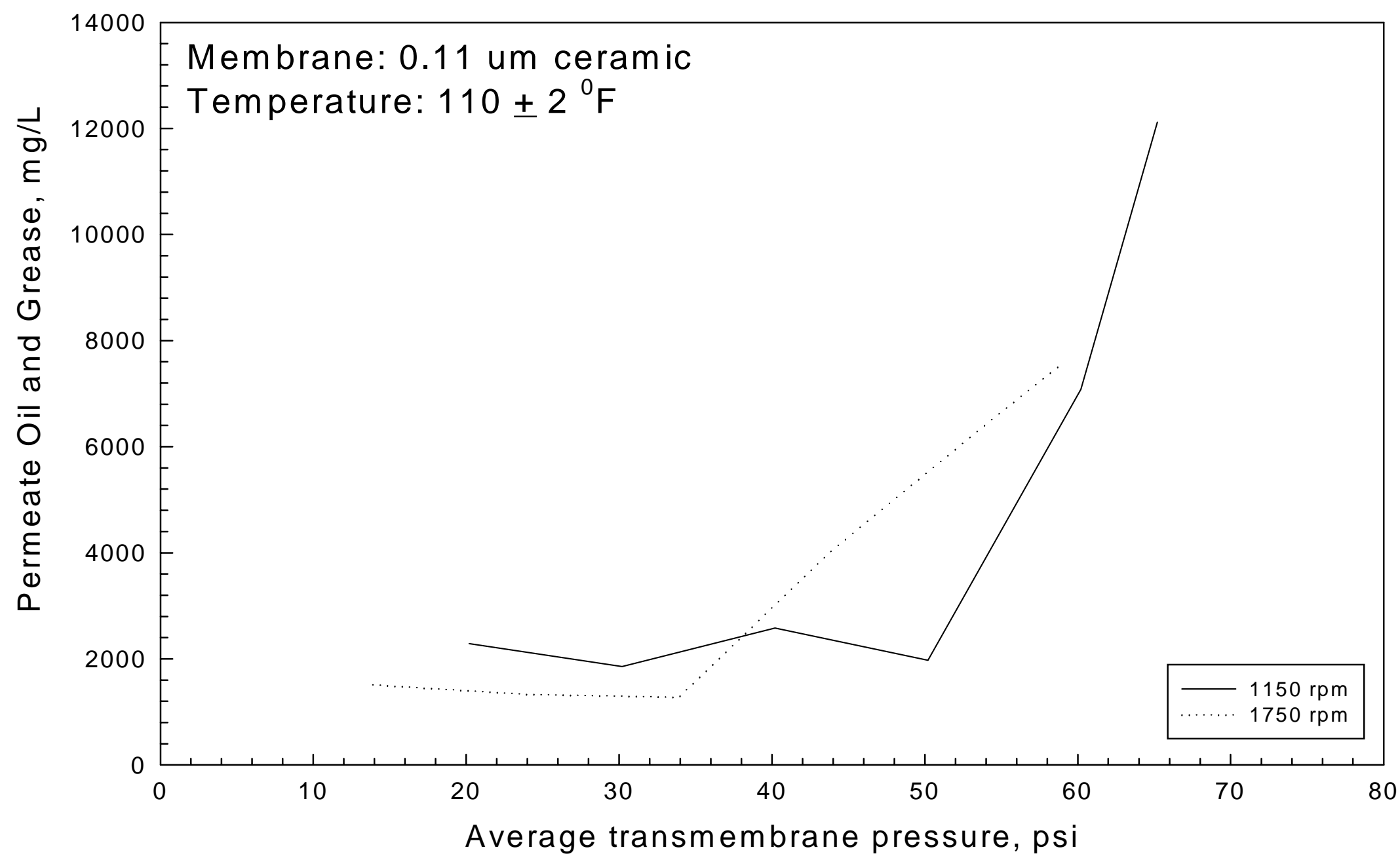

Figure (5.4)—Permeate Oil and Grease Versus Average Transmembrane Pressure For the 40\% MWF Combinations. 


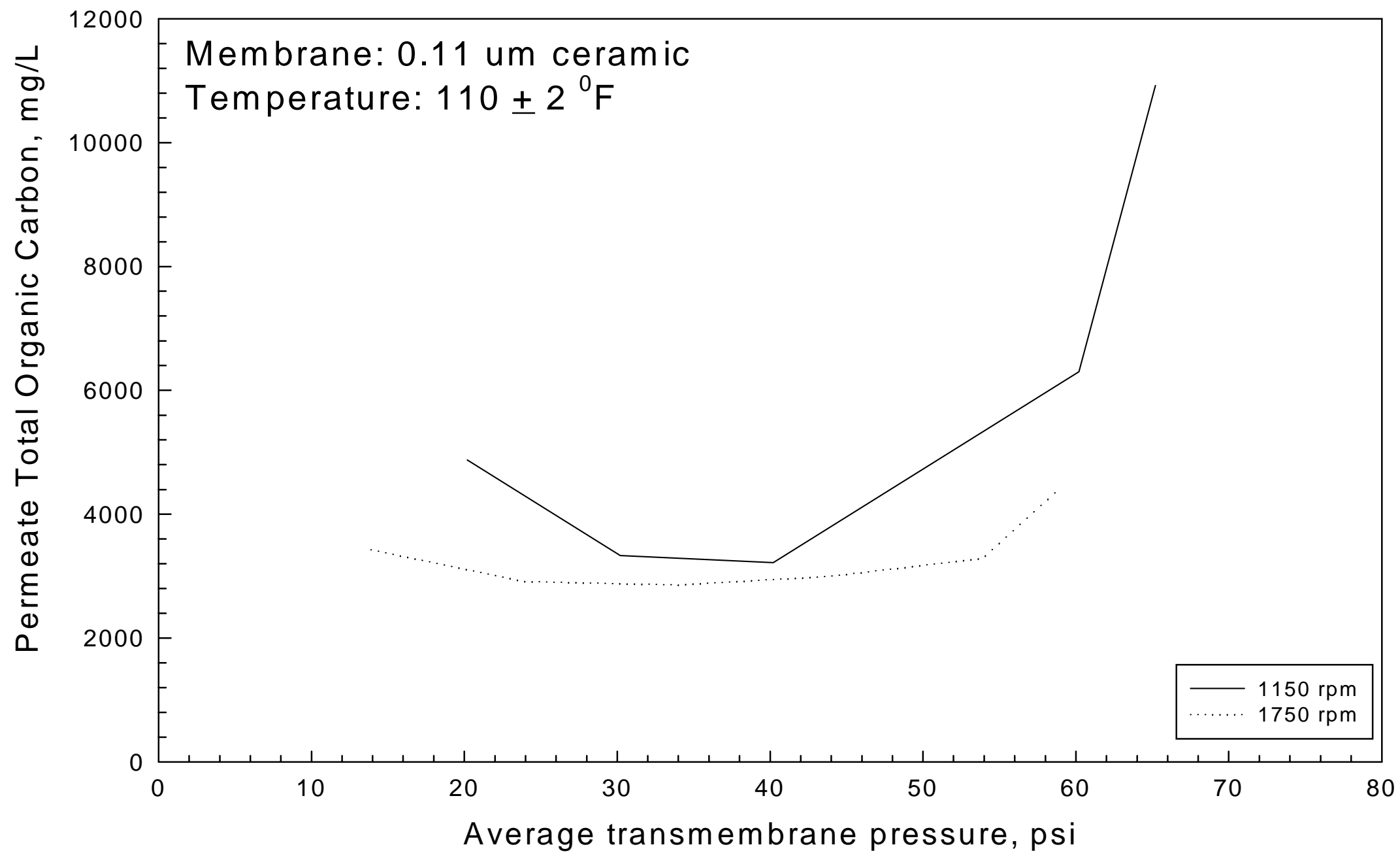

Figure (5.5)—Permeate Total Organic Carbon Versus Average Transmembrane Pressure For the 40\% MWF Combinations. 


\section{7). It is hypothesized that the lower concentrations of rejected oil droplets at the}

membrane surface resulted in a decreased probability of oil permeation through the membrane pores, and a decrease in both $\mathrm{O} / \mathrm{G}$ and TOC was observed.

Similar trends were observed in $\mathrm{O} / \mathrm{G}$ and $\mathrm{TOC}$ versus $\mathrm{OC}_{\mathrm{k}}$, TMP and $\mathrm{R}_{\mathrm{j}}$ throughout the experiments conducted in this research. However, $\mathrm{O} / \mathrm{G}$ trends were more definitive than those observed in TOC. It is hypothesized that low molecular weight additives in the MW fluid passed through the membrane and were measured as TOC. Therefore, changes in TOC versus $\mathrm{OC}_{k}$, TMP and $\mathrm{R}_{\mathrm{j}}$ were minimal and much less definitive than those observed in O/G.

In general, $\mathrm{O} / \mathrm{G}$ rejections were in excess of $99 \%$, while TOC rejections were in excess of $98 \%$. Both $\mathrm{O} / \mathrm{G}$ and TOC rejections were independent of each of the variables examined. Lipp et al. (1984) reported $\mathrm{O} / \mathrm{G}$ rejections in excess of $99.9 \%$ and TOC rejections in excess of 96\%, in the ultrafiltration of an oil-water emulsion. However, rejections are not necessarily a good representation of permeate water quality due to the fact that calculations are based on oil concentrations in the feed solution. For example, $500 \mathrm{mg} / \mathrm{L}$ of oil in the permeate observed from a 5\% feed solution results in a $99 \%$ rejection. Similarly, 4,000 mg/L of oil in the permeate observed from a $40 \%$ feed solution would also result in a 99\% rejection. Therefore, increasing concentrations of oil and other solutes in the permeate do not assure increases in rejection values. 


\section{CHAPTER 6.0 \\ CONCLUSIONS}

A study was performed using a synthetic MW fluid to examine the effects of feed oil content, transmembrane pressure, and membrane rotational speed on permeate water quality in the HSRUF system. In general, the lowest feed oil concentrations and transmembrane pressures, and the highest membrane rotational speeds produced the best permeate water quality. Sharp increases in both $\mathrm{O} / \mathrm{G}$ and TOC were observed from approximately $25-35 \%$ oil in the feed solution. These increases were attributed to similar increases in feed viscosity at the same oil contents. When permeate water quality is of main concern, it is most efficient to operate the lowest pressure and viscosity feasible, and the highest membrane rotational speed. However, permeate flux and operational costs must also be considered in most full-scale operations. Results from this study must be examined along with results from similar studies focusing on permeate flux. Furthermore, research on the economics of HSRUF operation is also necessary in order to maximize operational efficiency on the full-scale. 


\section{CHAPTER 7.0 \\ REFERENCES}

Belfort, G., Mikulasek, P., Pimbley, J., and K. Chung (1993). "Diagnosis of Membrane Fouling Using a Rotating Annular Filter, Part 1: Cell Culture Media," Journal of Membrane Science, 77, 1-22.

Belfort, G., Mikulasek, P., Pimbley, J., and K. Chung (1993). "Diagnosis of Membrane Fouling Using a Rotating Annular Filter, Part 2: Dilute Particle Suspensions of Known Particle Size," Journal of Membrane Science, 77, 23-39.

Belfort, G. (1988). "Membrane Modules: Comparison of Different Configurations Using Fluid Mechanics," Journal of Membrane Science, 35, 245-270.

Bennett, G. (1988). "The Removal of Oil From Wastewater By Air Flotation, A Review," CRC Critical Reviews in Environmental Control, 18 (3) 220.

Benson, R. (1994). "Processing Apparatus With Wall Conditioning Shuttles," US Patent Number 5,284,581.

Bhave, R. (1991). Inorganic Membranes: Synthesis, Characteristics, and Applications, Van Nostrand Reinhold, New York.

Bird, R., Stewart, W., and E. Lightfoot (1960). Transport Phenomena, John Wiley and Sons, New York.

Blatt, W., David, A., Michaels, A., and L. Nelson (1970). Membrane Science and Technology, J. Flinn, Ed., Plenum Press, New York.

Bodzek, M. and K. Konieczny (1992). "The Use of Ultrafiltration Membranes Made of Various Polymers In The Treatment of Oil-Emulsion Wastewaters," Waste Management, 12, 75-84.

Bowker, A. and G. Lieberman (1972). Engineering Statistics, $2^{\text {nd }}$ ed., Prentice Hall, Englewood Cliffs, New Jersey. 
Bryan, P. (1997). Personal Communication With Senior Process Engineer, Union Carbide Technical Center.

Burke, J. (1991). "Waste Treatment of Metal-Working Fluids, A Comparison of Three Common Methods," Lubrication Engineering, April, 238-246.

Canepa, P., Marignetti, N., Rognoni, U., and S. Calgari (1988). Olive Mills Wastewater Treatment By Combined Membrane Processes," Water Resources, 22 (12), 14911494.

Cheryan, M. (1977). "Mass Transfer Characteristics of Hollow Fiber Ultrafiltration of Soy Protein Systems," Journal of Food Process Engineering, 1, 269-287.

Cheryan, M. (1997). Ultrafiltration and Microfiltration Handbook, Technomic Publishing Co., Inc., Lancaster, Pennsylvania.

Chiang, B. (1982). Ph.D. Thesis, University of Illinois, Urbana, IL.

de Filipi, R. (1977). Filtration, Principles and Practices Part 1, C. Orr, Ed., MarcelDekker, New York.

Dolecek, P., Mikulasek, P., and G. Belfort (1995). “The Performance of a Rotating Filter. Theoretical Analysis of the Flow in an Annulus with a Rotating Inner Porous Wall," Journal of Membrane Science, 99, 241-248.

Elmaleh, S. and W. Naceur (1992). "Transport of Water Through an Inorgainc Composite Membrane," Journal of Membrane Science, 66, 227-234.

Eringis, A., Brady, F., and R. Blanck (1993). "Wastewater Treatment in the Die Casting Industry Using Cross-Flow Membrane Filtration," Transactions of the North American Die Casting Association.

Fane, A. and C. Fell (1987). "A Review of Fouling and Fouling Control in Ultrafiltration," Desalination, 62, 117-136. 
Goldsmith, R., Roberts, D., and D. Burre (1974). "Ultrafiltration of Soluble Oil Wastes," Journal of The Water Pollution Control Federation, 46 (9), 2183-2192.

Gutman R. (1987). Membrane Filtration: The Technology of Pressure-Driven Membrane Processes, IOP Publishing, Ltd., Bristol, Great Britain.

Isaacson, K., Duenas, P., Ford, C., and M. Lysaght (1980). "Polymer Science and Technology," 13, 507.

Jonsson, G. and C. Boesen (1984). "Polarization Phenomena in Membrane Processes," Synthetic Membrane Processes, G. Belfort, Ed., Academic Press, Inc., New York.

Jonsson, A. and G. Tragardh (1990). "Fundamental Principles of Ultrafiltration," Chemical Engineering Processes, 27, 67-81.

Ketola, H. and J. McGrew (1968). "Pressure, Frictional Resistance, and Flow Characteristics of the Partially Wetted Rotating Disk," Journal of Lubrication Technology, April, 395-404.

Klinestreuer, C. and G. Belfort (1984). "Mathematical Modeling of Fluid Flow and Solute Distribution in Pressure-Driven Membrane Modules," Synthetic Membrane Processes, G. Belfort, Ed., Academic Press, Inc., New York.

Kozinski, A and E. Lightfoot (1972). American Institute of Chemical Engineering Journal, 18, 1030.

Laemmle, J. (1992). Metalworking Lubricants, American Petroleum Institute.

Lee, S., Aurelle, Y., and H. Roques (1984). "Concentration Polarization, Membrane Fouling, And Cleaning In Ultrafiltration Of Soluble Oil," Journal Of Membrane Science, 19, 23-38.

Liberherr, J. (1978). Scherifiltration im Ringspalt, Ph.D. Thesis, ETH, Zurich. 
Lipp, P., Lee, C., Fane, A., and C. Fell (1988). "A Fundamental Study of The Ultrafiltration of Oil-Water Emulsions," Journal of Membrane Science, 36, 161177.

Lopez-Leiva (1980). "Ultrafiltration at Low Degrees of Concentration Polarization: Technical Possibilities," Desalination 35, 115-128.

MacNeil, J. and D. McCoy (1989). "Membrane Separation Technologies," Standard Handbook of Hazardous Waste Treatment and Disposal, McGraw-Hill Book Co., New York, 6.91-6.106.

Mahdi, S. and R. Skold (1991). "Membrane Filtration for the Recycling of Water-Based Synthetic Metalworking Fluids," Filtration and Separation, 28 (6), 407-414.

Mir, L., Eykamp, W., and R. Goldsmith (1977). "Current and Developing Applications for Ultrafiltration," Industrial Water Engineering, May/June, 14-19.

Murkes, J. and C. Carlsson (1988). Crossflow Filtration, John Wiley and Sons, Ltd., Bath, Great Britain.

Nazzal, F. and M. Wiesner (1996). "Microfiltration of Oil-in-Water Emulsions," Water Environment Research, 68 (7), 1187-1191.

Perry, R. and D. Green (1997). Perry's Chemical Engineering Handbook, $7^{\text {th }}$ ed., McGraw-Hill, New York.

Pinto, S. (1978). "Ultrafiltration for Dewatering of Waste Emulsified Oils," Proceedings, $I^{\text {st }}$ International Conference, IIT Research Institute, Chicago, Illinois, 1-4.

Porter, M. (1979). Handbook of Separation Techniques for Chemical Engineers, P. Schweitzer, Ed., McGraw-Hill, New York.

Porter, M. and A. Michaels (1972). Chemical Technology, 2, 56. 
Reed, B., Lin, W., Viadero, R., and J. Young (1997). "Treatment of Concentrated Oily Wastes Using Centrifugal Membrane Separation (CMS)," ASCE Journal of Environmental Engineering, 123 (12), 1-9.

Reed, B., Lin, W., Dunn, C., Carriere, P., and G. Roark (1997). "Treatment of an Oil/Grease Wastewater Using Ultrafiltration: Pilot-Scale Results," Separation Science and Technology, 32 (9), 1493-1511.

Singh, R. (1996). "Investigation of Ultrafiltration Rejection of Surfactant Micelles by Dynamic Light Scattering," Separation Science and Technology, 31 (9), 13511356.

Smith, A. and S. Circle (1972). Soybeans: Chemistry and Technology, Avi Publishing Co., New York.

Snoeyink, V. and D. Jenkins (1980). Water Chemistry, John Wiley and Sons, New York.

Spriggs, H. and N. Li (1976). "Liquid Permeation Through Polymeric Membranes," Membrane Separation Processes, P. Meares, Ed., Elsevier Publishing Co, New York.

Standard Methods for the Examination of Water and Wastewater. (1985). American Public Health Association (APHA), Washington, D.C.

Viadero, R. (1997). "Mass-Transfer Limited Permeate Flux in High-Shear Rotary Ultrafiltration: Effects of Transmembrane Pressure and Membrane Rotational Speed," Ph.D. Dissertation, West Virginia University, Morgantown, WV.

Vigo, F., Uliana, C., and P. Lupino (1985). "The Performance of a Rotating Module in Oily Emulsions Ultrafiltration," Separation Science and Technology, 20 (2\&3), 213-230.

Yan, S., Hill, C., and C. Amundson (1979). Journal of Dairy Science, 62, 23.

Weast, R., Ed. (1976). Handbook of Chemistry and Physics, CRC Press, Cleveland, OH. 
Woerner, D. (1983). Ph.D. Thesis, University of Washington.

Zaidi, A., Simms, K., and S. Kok (1992). "The Use of Micro/Ultrafiltration for the Removal of Oil and Suspended Solids From Oilfield Brines," Water Science and Technology, 25 (10), 163-176. 